\title{
UTILIZACIÓN DE REDES DE SIETE PUERTOS EN SISTEMAS CON DIVERSIDAD POLARIMÉTRICA Y ESPACIAL
}

\author{
Tesis de Doctorado
}

\section{ALEJANDRO J. VENERE}

Presentada ante la Facultad de Ingeniería de la Universidad Nacional de La Plata como requisito para la obtención del grado académico de

DOCTOR EN INGENIERIA

Dirección de tesis:

Dr. Ing. Martín Hurtado

Jurado de tesis:

Ing. Hugo Lorente

Dr. Ing. Mario Hueda

Dr. Ing. Fernando Gregorio 



\section{Resumen}

La tecnología inalámbrica moderna utiliza arreglos de sensores para incorporar información polarimétrica y espacial del entorno proporcionando funciones adicionales a los sistemas como la capacidad de transmitir y recibir señales con distinta polarización, así como también, conformar el haz de transmisión y localizar fuentes de señal en el receptor. En general, un arreglo de sensores consiste en varias antenas cada una asociada a un receptor o transmisor heterodino en cuadratura, todos conectados al mismo oscilador de referencia. Debido al incremento del número de antenas y el aumento constante de aplicaciones en el espectro de las microondas y ondas milimétrica, la impelmentación de estos sistemas resulta cada vez más compleja. Por este motivo, se fomenta la búsqueda de nuevas arquitecturas eficientes que permitan operar un arreglo de sensores manteniendo circuitos simples, de bajo consumo de potencia y fácil integración con las restantes partes del sistema. En la actualidad, una alternativa que ha desplazado casi por completo a las arquitecturas convencionales basadas en esquemas heterodinos, son los dispositivos de seis puertos propuestos a mediados de 1990 como una forma simple y económica de diseñar demoduladores y moduladores en alta frecuencia. La ventaja principal de los dispositivos de seis puertos, además del bajo costo y consumo de energía del circuito, es el gran ancho de banda y pequeño tamaño que se consiguen en frecuencias de microondas y ondas milimétricas.

Utilizando el concepto de seis puertos, en esta tesis se presentan los fundamentos teóricos y la implementación de arquitecturas basadas en una estructura de siete puertos. Los esquemas propuesto permiten integrar dos antenas a una única estructura de siete puertos logrando reducir el costo, el consumo y el tamaño de los circuito respecto a implementaciones basadas en dispositivos de seis puertos. En primer lugar, se presenta el diseño de un sistema de transmisión de siete puertos capaz de modular dos señales de salida a partir de cuatro impedancias variables controladas por señales de banda base. Este modulador se implementa junto con una antena de dos puerto para obtener un sistema capaz de modular la envolvente de la señal transmitida y configurar la polarización de la antena. Se analizan las características del modulador respecto a los dispositivos de seis puertos y se validan las expresiones teóricas utilizando un prototipo.

Otra de las arquitecturas desarrolladas consiste en un demodulador de siete puertos capaz de recuperar las envolventes complejas de las señales recibidas por tres antenas utilizando únicamente tres detectores de potencia. Se plantea un esquema de demodulación heterodino basado en la transformada de Hilbert a partir del cual se reduce el costo de construcción y el consumo de energía del dispositivo a costa de aumentar la complejidad del procesamiento digital. Los dos dispositivos, el demodulador y el modulador, fueron validados a partir de prototipos implementados para operar a una frecuencia de $1575,42 \mathrm{MHz}$ correspondiente a la banda L1 de GPS. Los resultados experimentales mostraron un buen 
comportamiento de los mismos respecto a los modelos teóricos desarrollados. 


\section{Agradecimientos}

A las instituciones que financiaron la realización de esta tesis: el Consejo Nacional de Investigaciones Científicas y Técnicas y la Universidad Nacional de La Plata. Al Instituto LEICI por proporcionar el lugar y material de trabajo y a todos sus integrantes que de una forma u otra fueron parte importante de este trabajo.

Al grupo de Procesamiento estadístico de señales y al grupo SENyT por acompañar, participar y brindar ideas durante todas las etapas realizadas.

A mi director Martín Hurtado, por su disponibilidad durante todo este periodo, por la confianza y el apoyo brindados desde un principio. También a Ramón Lopez la Valle que junto a Martín Hurtado fue director de mi trabajo final de carrera, donde comencé a tomar contacto con los temas abordados en esta tesis.

Por último no puedo dejar de agradecer a mis amigos, a los compañeros de Purparlé, a mi esposa Nabila y por sobre todo a mi familia por el apoyo incondicional. 



\section{Índice general}

Resumen ........................... I

Agradecimientos .......................... III

1. Introducción 1

1.1. Sistemas Inalámbricos . . . . . . . . . . . . . . . . 1

1.1.1. Técnicas de Diversidad . . . . . . . . . . . . . . . . . . . 1

1.2. Motivación y Objetivos . . . . . . . . . . . . . . . . . 3

1.2.1. Arquitecturas Convencionales . . . . . . . . . . . . . 3

1.2.2. Arquitecturas de Seis Puertos . . . . . . . . . . . . . 3

1.2.3. Arquitecturas Propuestas ... . . . . . . . . . . . 4

1.3. Contribuciones Originales . . . . . . . . . . . . . . . 4

1.4. Organización de la Tesis . . . . . . . . . . . . . . . . 6

2. Técnicas Seis Puertos 7

2.1. Antecedentes de Seis Puertos . . . . . . . . . . . . . . . 7

2.2. Red de Seis Puertos . . . . . . . . . . . . . . . . . . . . . 7

2.2.1. Modelo Teórico . . . . . . . . . . . . . . . . . . . . 8

2.3. Demodulador de Seis Puertos . . . . . . . . . . . . . . . . . . 12

2.4. Modulador de Seis Puertos . . . . . . . . . . . . . . . . . . . 16

2.5. Esquema con Múltiples Antenas . . . . . . . . . . . . . . . . . . . . . . . . 19

2.5.1. Representación de la Polarización . . . . . . . . . . . . . . . . . . . 19

2.5.2. Representación del Ángulo de Arribo . . . . . . . . . . . . . . . . . 20

3. Modulador de Siete Puertos 22

3.1. Descripción del Dispositivo . . . . . . . . . . . . . . . . . 22

3.1.1. Modelo Teórico . . . . . . . . . . . . . . . . . . . 22

3.1.2. Ejemplos de Aplicación . . . . . . . . . . . . . . . . . 26

3.2. Descripción del Prototipo . . . . . . . . . . . . . . . . . . 29

3.2.1. Red de Siete Puertos . . . . . . . . . . . . . . . . . . . 29 
3.2.2. Impedancias Variables $\ldots \ldots \ldots . . \ldots \ldots$

3.2.3. Antena . . . . . . . . . . . . . . . . . . . . . . . 33

3.3. Resultados . . . . . . . . . . . . . . . . . . . 33

3.3.1. Validación del Modelo . . . . . . . . . . . . . . . . . 34

3.3.2. Validación del Prototipo . . . . . . . . . . . . . . . 35

3.3.3. Validación con Antenas . . . . . . . . . . . . . . . . . . 36

3.4. Ventajas y Desventajas . . . . . . . . . . . . . . . . . 37

4. Impedancias Variables 40

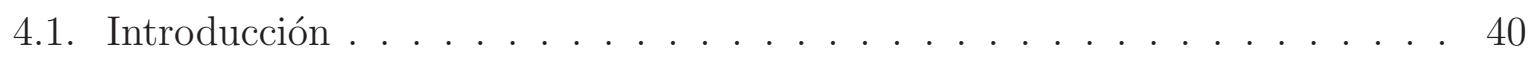

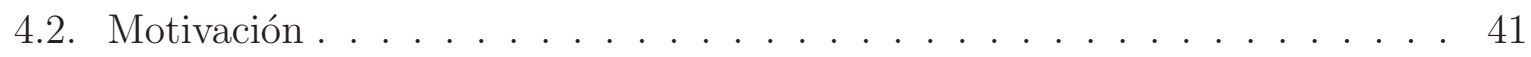

4.3. Propuesta Basada en dos Diodos PIN . . . . . . . . . . . . . . . . 41

4.3.1. Modelo Analítico del Coeficiente de Reflexión . . . . . . . . . . 42

4.4. Implementación del Circuito . . . . . . . . . . . . . . . . 43

4.5. Resultados . . . . . . . . . . . . . . . . . . . 44

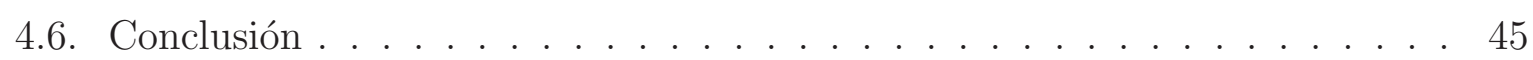

5. Procedimiento de Calibración 46

5.1. Introducción . . . . . . . . . . . . . . . 46

5.1.1. Formulación del Problema . . . . . . . . . . . . . . . . . . 47

5.2. Descripción del Método de Mapa de Difusión . . . . . . . . . . . . . . . 47

5.2.1. Kernel de Difusión . . . . . . . . . . . . . . . . . . . . . 47

5.2.2. Descomposición . . . . . . . . . . . . . . . . . . . . 48

5.2.3. Parámetro de Escala . . . . . . . . . . . . . . . . . . . . . . 49

5.3. Implementación del Método . . . . . . . . . . . . . . . . . . . . . 49

5.3.1. Ejemplo Simulado . . . . . . . . . . . . . . . . . . 50

5.3.2. Ejemplo Medido. . . . . . . . . . . . . . . . . . 52

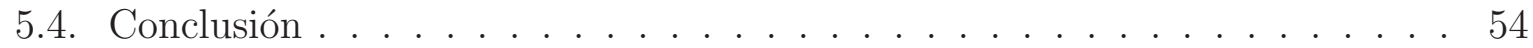

6. Demodulador de Multiples Puertos $\quad 56$

6.1. Introducción . . . . . . . . . . . . . . . 56

6.2. Fundamentos Teóricos . . . . . . . . . . . . . . . . . 57

6.2.1. Modelo Analítico . . . . . . . . . . . . . . . . . 57

6.2.2. Ecuaciones de Demodulación . . . . . . . . . . . . . . . . . 61

6.2.3. Demodulador de Siete Puerto I . . . . . . . . . . . . . . . 62

6.2.4. Transformada de Hilbert . . . . . . . . . . . . . . . . . . 64

6.2.5. Validación Experimental . . . . . . . . . . . . . . . . 65 
6.3. Demodulador de Siete Puertos II . . . . . . . . . . . . . . . . . . 68

6.3.1. Modelo Teórico . . . . . . . . . . . . . . . . . . . . . 68

6.3.2. Validación Experimental . . . . . . . . . . . . . . . . . . . 70

6.4. Conclusión . . . . . . . . . . . . . . . . . . . . 71

$\begin{array}{ll}\text { 7. Conclusiones } & 73\end{array}$

$\begin{array}{ll}\text { A. Eficiencia de Conversión en Función de } \theta & 76\end{array}$

B. Eficiencia de Conversión en Función de $\beta \quad 77$

C. Calibración del Modulador de Siete Puertos 78

D. Expresión de la Salida del Filtro $\quad 80$ 


\section{Índice de figuras}

1.1. Esquema básico de un canal inalámbrico. . . . . . . . . . . . . . 2

1.2. Esquema heterodino básico de un (a) receptor, y (b) un transmisor. . . . . 3

1.3. Esquema de un (a) receptor y (b) un transmisor basados en una estructura de seis puertos. . . . . . . . . . . . . . . . . . 4

2.1. Redes de múltiples puertos utilizadas en el diseño de moduladores y demoduladores de seis puertos $[1] \ldots \ldots \ldots$. . . . . . . . . . . 8

2.2. Dispositivo de un puerto conectado a una fuente de señal . . . . . . . . . . 9

2.3. Dispositivo de $N$ puerto conectado a una fuente de señal en el puerto 1 . . 10

2.4. Esquema de un (a)Divisor Wilkinson y un (b) Acoplador Híbrido en cuadratura . . . . . . . . . . . . . . . . . . 10

2.5. Diagrama de grafo establecido cuando se excita el puerto $P_{1} \ldots \ldots$. . . 11

2.6. Teoría de grafos, ramas en serie $[2] \ldots \ldots$. . . . . . . . . . . . 12

2.7. Demodulador basado en una red de seis puertos. . . . . . . . . . . . . . 13

2.8. Modulador basado en una red de seis puertos. . . . . . . . . . . . . 17

2.9. Elipse de polarización . . . . . . . . . . . . . . . . . . . 20

2.10. Modelo geométrico . . . . . . . . . . . . . . . . . . . 21

3.1. Modulador basado en una red de siete puertos. . . . . . . . . . . . . . 23

3.2. Eficiencia teórica en función del ángulo $\theta \ldots$. . . . . . . . . . . . 27

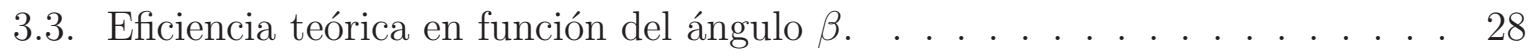

3.4. Prototipo implementado para operar a la frecuencia 1575,42 MHz. Modulador completo (izquierda), cargas variables (derecha). . . . . . . . . 29

3.5. Magnitud de los parámetros $\mathrm{S}$ de la red de siete puertos. . . . . . . . . . 30

3.6. Circuito de las cargas variables . . . . . . . . . . . . . . 30

3.7. Simulación de $\Gamma_{d}(I)$ para diferentes inductancias parásitas . . . . . . . . 31

3.8. Circuito de las cargas variables compensadas . . . . . . . . . . . . . 32

3.9. Coeficientes de reflexión medidos a 1575,42 MHz. . . . . . . . . . . . . . 33

3.10. Antena implementada y simulada . . . . . . . . . . . . . . . 34 
3.11. Parámetros $\mathrm{S}$ medidos y simulados de la antena de dos puertos, $P_{V}=P_{1}$

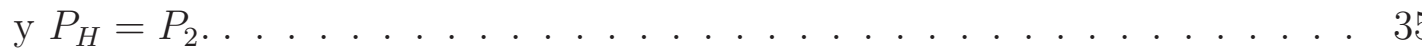

3.12. Experiencia de medición, configuración y conexión . . . . . . . . . 36

3.13. Respuesta de las salidas del modulador de siete puertos: Resultados teóricos (o rojo), resultados medidos en la salida (* azul) y resultados medidos con antenas ( $\diamond$ negro). (a) Elipticidad $\beta$, (b) orientación $\alpha,(\mathrm{c})$ envolvente compleja, (d) eficiencia. . . . . . . . . . . . . . . . . .

3.14. Respuesta de salida del modulador de siete puertos a 1575,42 MHz. Valores deseados (o rojo) y valores obtenidos de las mediciones (* azul) de (a) la envolvente compleja, (b) la orientación $\alpha$, y (c) la elipticidad $\beta . . .38$

3.15. Conexión de las salidas del modulador para la medición con antenas. . . . 39

4.1. Esquema básico del circuito propuesto . . . . . . . . . . . . . . . . . 41

4.2. Valores de impedancia mapeados en el diagrama de Smith. Resultados simulados a partir del modelo (4.8) variando $I_{2}$ e $I_{3}$ en el rango $[0,40] \mathrm{mA}$, (a) sin considerar inductancia parásita $(L=0 \mathrm{H})$, (b) con inductancia parásita $(L=1,5 \mathrm{nH}) \ldots \ldots \ldots \ldots \ldots \ldots$

4.3. Circuito esquemático del prototipo propuesto . . . . . . . . . . . . . . . 43

4.4. Valores de impedancia mapeados en el diagrama de Smith cuando las corrientes $I_{2}$ e $I_{3}$ varían entre $[0,40] \mathrm{mA}$. Resultados medidos (a) antes de incluir el inductor de compensación, y (b) para distintas frecuencias de operación e incluyendo la compensación.

5.1. Valores simulados del coeficiente de reflexión (4.7) mapeados en el diagrama de Smith. Resultados de los datos de entrenamiento y los valores deseados.

5.2. Error medio de validación obtenido a partir de observaciones de entrenamiento en función de la escala $\varepsilon$, a partir de diferentes valores de la constante de varianza $k \ldots \ldots \ldots \ldots \ldots$. . . . . . . . . . . .

5.3. Respuesta simulada de la impedancia variable mapeada en el gráfico de Smith. Observaciones deseadas y observaciones obtenidas a partir de la simulación. . . . . . . . . . . . . . . . . . .

5.4. Respuesta medida de la impedancia variable del Capítulo 4 a 1575,42 MHz. Observaciones de deseadas y observaciones obtenidas de las mediciones. . .

5.5. Error medio de validación en función de la escala $\varepsilon$ del kernel gaussiano calculado para todos los datos de entrenamiento del prototipo. . . . . . . .

5.6. Respuesta medida del prototipo de la impedancia variable mapeada en el gráfico de Smith. Observaciones deseadas y observaciones obtenidas a partir de las mediciones. . . . . . . . . . . . . . . . . . . . . 55

6.1. Demodulador de $\mathrm{N}$ puertos. . . . . . . . . . . . . . . 57

6.2. Magnitud del espectro de la señal de salida $y_{i}(t) \ldots \ldots$. . . . . . . . 61

6.3. Demodulador basado en una red de siete puertos. . . . . . . . . . . . . 63 
6.4. Fotografía del prototipo implementado para operar a 1575,42 MHz: red de siete puertos (izquierda) y detector de potencia (derecha). . . . . . . . . 65

6.5. Diagrama del sistema de medición. . . . . . . . . . . . . . . 66

6.6. Resultados medidos. Magnitud y fase de las envolventes complejas $x_{2}[n]$ y $x_{3}[n]$ para los casos $(\mathrm{a})-(\mathrm{d}) . \ldots \ldots \ldots . \ldots . \ldots . \ldots 67$

6.7. Demodulador basado en una red de siete puertos. . . . . . . . . . . . . 69

6.8. Diagrama del sistema de medición. . . . . . . . . . . . . . 70

6.9. Resultados medidos. Magnitud y fase de las envolventes complejas $x_{2}[n]$, $x_{3}[n]$ y $x_{4}[n]$ para el caso $(\mathrm{a})$ y $(\mathrm{b}) \ldots \ldots \ldots$. . . . . . . . . . . 71 


\section{Capítulo 1}

\section{Introducción}

En este capítulo se realiza una breve introducción a los sistemas inalámbricos, haciendo hincapié en las aplicaciones con diversidad polarimétrica y espacial. Luego se exponen las razones que motivaron la utilización de redes de siete puertos en el diseño de receptores y transmisores. Finalmente, se describe la organización de la tesis.

\subsection{Sistemas Inalámbricos}

Casi todos los aspectos de la vida moderna se basan en información recopilada por tecnologías inalámbricas. Esta tecnología ha evolucionado a lo largo de los años desde los primeros intentos de transmitir un mensaje a través del Atlántico por Marconi en 1901, hasta los últimos desarrollos en aplicaciones de telefonía móvil, sistema de navegación, sistemas de transporte inteligente (ITS) e identificación por radio frecuencia (RFID). Estos son solo algunos de los nombres que actualmente impulsan la revolución inalámbrica y tienen una gran influencia en nuestro estilo de vida.

En aspectos generales, un sistema inalámbrico opera transmitiendo y recibiendo información a través del espacio libre. Esta tarea es posible por el hecho de que las ondas electromagnéticas se propagan en el espacio sin la necesidad de una conexión por cable. Una característica fundamental del medio inalámbrico es que la señal transmitida puede propagarse en múltiples direcciones, recorrer diferentes caminos, e interactuar con múltiples obstáculos antes de alcanzar el receptor, como se muestra en la Figura 1.1. Esta interacción con el medio es fundamental en aplicaciones de sensado remoto donde el sistema obtiene información del entorno a partir de las reflexiones de las ondas electromagnéticas. Por el contrario, las características de multicamino degradan enormemente el funcionamiento de los sistemas de comunicaciones inalámbricos debido a los efectos de desvanecimiento [3].

\subsubsection{Técnicas de Diversidad}

Mediante técnicas de diversidad es posible reducir la probabilidad de desvanecimiento en los sistemas de comunicaciones. Esta técnica permite explotar la naturaleza dinámica del medio inalámbrico combinando o conmutando réplicas de la señal original, producidas en diferentes intervalos de tiempo, bandas de frecuencia, espacio y estados de polarización. 


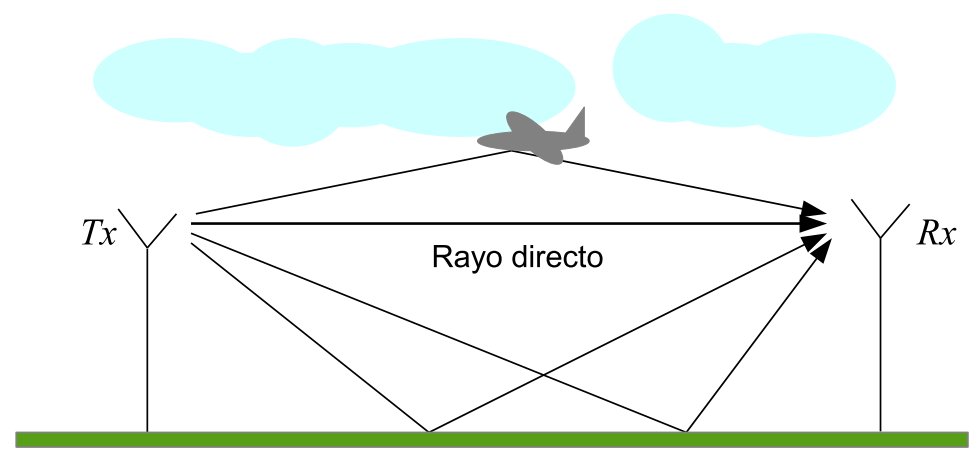

Figura 1.1: Esquema básico de un canal inalámbrico.

Si varias réplicas de la señal se reciben a través de enlaces independientes, habrá una alta probabilidad de que uno o más de los enlaces no sufran desvanecimiento. Esta probabilidad aumentará si el número de ramas de diversidad aumenta. Por ejemplo, para incrementar la diversidad en el tiempo se necesita mas tiempo para transmitir mayor número de replicas, en consecuencia se reduce la tasa de datos. Asimismo, la diversidad en frecuencia aumenta a costa de incrementar el ancho de banda del sistema. En la actualidad, la tecnología inalámbrica utiliza múltiples antenas o antenas inteligentes para incorporar información polarimétrica y espacial de las señales electromagnéticas permitiendo aumentar el grado de diversidad de las señales sin modificar los requerimientos de tasa de datos y ancho de banda.

\section{Diversidad Polarimétrica}

La diversidad polarimétrica es una técnica que permite caracterizar el campo electromagnético incidente discriminando las fuentes de señal según su polarización. Esta técnica se logra empleando múltiples antenas o antenas de doble polarización [4, 5] las cuales permiten transmitir y/o recibir señales con distinta polarización. En general, se utilizan combinaciones de polarización lineal vertical y horizontal, o circular izquierda y derecha. La información de polarización se utiliza en múltiples aplicaciones inalámbricas, como por ejemplo en sistemas de comunicaciones [6, 7], radar metorológico [8], radar de apertura sintética polarimétrico [9, 10], y RFID [11].

\section{Diversidad Espacial}

La diversidad espacial se obtiene colocando antenas en distintas posiciones para adquirir muestras temporales y espaciales del campo electromagnético en el cual se encuentra inmerso. A diferencia de lo que ocurre en la recepción con una sola antena, el uso de múltiples antenas provee información adicional que permite mejorar la relación de señal a ruido, separar distintas fuentes de señal y cancelar interferencias [12]. Una aplicación importante es caracterizar el campo electromagnético incidente determinando el número de fuentes, su ubicaciónón y la señal emitida $[13,14]$. 


\subsection{Motivación y Objetivos}

El incremento del número de antenas requerida en los sistemas modernos, y el aumento constante de aplicaciones inalámbricas en el espectro de las microondas, fomentan la búsqueda de nuevas arquitecturas eficientes que permitan operar con múltiples antenas manteniendo circuitos simples, de bajo consumo de potencia y fácil integración con las restantes partes del sistema.

\subsubsection{Arquitecturas Convencionales}

Las arquitecturas más utilizadas debido a sus caracterísitcas de sensibilidad, selectividad e inmunidad al ruido son los receptores y transmisores heterodinos mostrados en la Figura 1.2. En este esquema se realizan dos o más conversiones de frecuencia. En el proceso de demodulación o modulación, la señal de banda base se divide en dos canales, llamados canal en fase I y canal en cuadratura Q, a partir de los cuales se define la envolvente compleja de la señal de interés [15]. Desbalances de ganancia y fase entre los canales I y Q suelen ser una fuente de error importante. Estos dispositivos deben usar filtros pasabanda para eliminar ruido y armónicos de orden superior de las bandas laterales de frecuencia, aumentando el costo de implementación y el tamaño del circuito. Además este tipo de arquitectura tiene un alto consumo de potencia dado por la cantidad de mezcladores que se usan en las etapas de conversión de frecuencia. Dada la complejidad

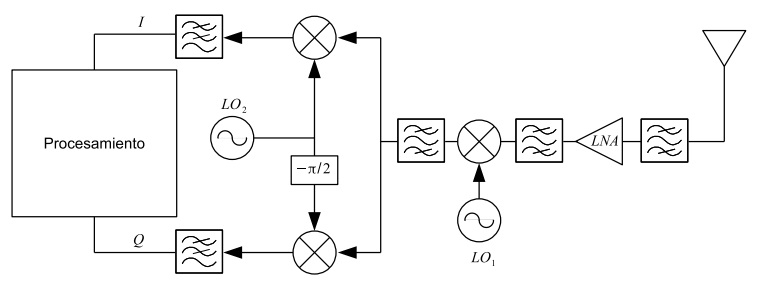

(a)

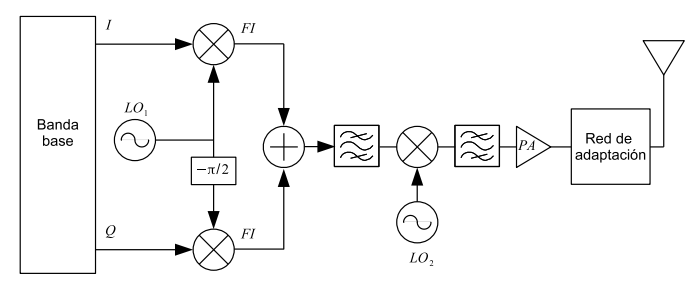

(b)

Figura 1.2: Esquema heterodino básico de un (a) receptor, y (b) un transmisor.

de los circuitos heterodinos, una alternativa conveniente consiste en el esquema homodino en el cual la señal recibida se traslada directamente a la banda base, donde la señal es filtrada y procesada en el dominio digital. De esta forma, se evitan los filtros pasabanda anteriores y los mezcladores adicionales reduciendo el costo y el consumo del circuito. En la práctica, los receptores homodinos son poco utilizados debido a los inconvenientes en conseguir alta ganancia y buena selectividad por filtrado directo de la señal. Asimismo, los esquemas de transmisión homodinos presentan problemas de fuga de portadora e intermodulación debido a la falta de filtrado en frecuencias intermedias y a la baja aislación entre el oscilador local y la salida.

\subsubsection{Arquitecturas de Seis Puertos}

Una alternativa eficiente para implementar el esquema homodino surge de la arquitectura de seis puertos. En un receptor basado en una arquitectura de seis puertos la 
conversión de frecuencia se produce directamente midiendo la potencia de las señales de salida, las cuales son combinaciones lineales de la señal recibida y el oscilador local. Esta operación se conoce como mezclador aditivo. El esquema de un receptor de seis puertos se muestra en la Figura 1.3(a). Este está formado por un circuito pasivo de seis puertos conectado a cuatro detectores de potencia a partir de los cuales se obtiene la envolvente compleja de la señal recibida. Por otro lado, un transmisor de seis puertos modula la amplitud compleja de la señal del oscilador de referencia utilizando impedancias variables controladas por un circuito de banda base, como muestra la Figura 1.3(b). Las ventajas

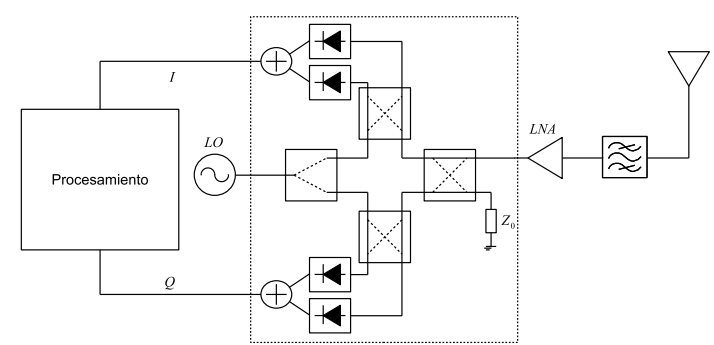

(a)

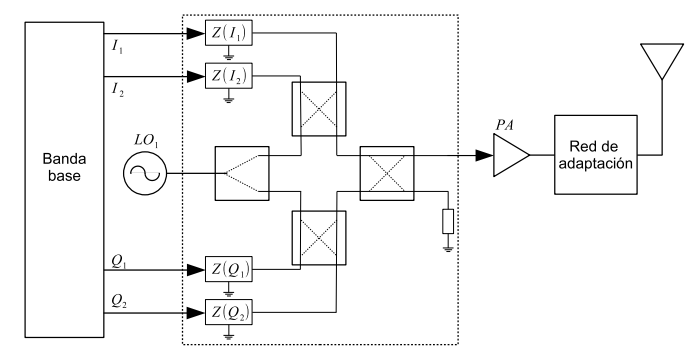

(b)

Figura 1.3: Esquema de un (a) receptor y (b) un transmisor basados en una estructura de seis puertos.

de estos esquemas de seis puertos son varias: en primer lugar, los dispositivos pueden ser completamente pasivos, contribuyendo a un muy bajo consumo de potencia. Además, dada la presencia del circuito pasivo de múltiples puertos se pueden lograr anchos de banda muy grandes y tamaño pequeños en frecuencias de microondas y ondas milimétricas. Por otro lado, en el receptor la mezcla entre las señales es aditiva y no requiere de altos niveles del oscilador local. El modulador de seis puertos permite reducir los problemas de fuga de portadora seleccionando adecuadamente la configuración de las impedancias variables $[16]$.

\subsubsection{Arquitecturas Propuestas}

La necesidad de utilizar sistemas con múltiples antenas incrementa rápidamente la complejidad del hardware debido a que aumenta el número de receptores y transmisores necesarios [13]. En esta tesis se utilizan estructura de siete puertos para la implementación de receptores y transmisores. Los dispositivos propuestos son una variante de las arquitecturas de seis puertos que permite conectar dos antenas y un oscilador local a la misma estructura, logrando circuitos de menor costo de construcción y menor consumo de energía respecto a implementaciones basadas en dispositivos de seis puertos.

\subsection{Contribuciones Originales}

Las contribuciones de esta tesis se dividen en dos partes principales. En primer lugar, se presenta el diseño de un modulador de siete puertos el cual se compone de 
cuatro cargas variables y una red pasiva de siete puertos; en segundo lugar, el diseño de un demodulador de siete puertos. Las arquitecturas propuestas brindan la posibilidad de conectar dos antenas al mismo hardware y por lo tanto, plantean una nueva alternativa para desarrollar sistemas con diversidad polarimétrica y espacial.

En primer lugar, se realizó el diseño de un modulador de siete puertos cuya finalidad es transmitir señales electromagnéticas con diferente polarización. El dispositivo propuesto está formado por una red de siete puertos y cuatro impedancias variables. Se desarrolló el modelo teórico que representa la señal transmitida en función de los valores de impedancia de las cargas variables y los parámetros del modelo de la red. Se desarrolló un prototipo de la red de siete puertos y se obtuvieron resultados preliminares midiendo la transferencia entrada-salida del modulador utilizando impedancias comerciales de valor fijo. Los fundamentos teóricos y los resultados fueron presentados en el trabajo Síntesis de Polarización con Redes de Siete Puertos que fue presentado en la XVI reunión de trabajo en Procesamiento de la Información y Control (RPIC), en la ciudad de Córdoba en Octubre de 2015 [17].

Dada la influencia de las impedancias variables en las características del modulador se estudiaron distintos diseños para las mismas. En primer lugar, para minimizar el número de señales de control se optó por un diseño simple donde cada impedancia variable está compuesta por un diodo PIN controlado por corriente. A partir de este diseño, se propuso otra arquitectura mas compleja para mejorar las características del modulador. Esta última se compone de dos diodos PIN y un divisor Wilkinson. El dispositivo propuesto fue validado con un prototipo y publicado en el trabajo New Design of Variable Impedance Based on Polarized Diodes at Microwave Frequencies el cual fue aceptado para su publicación en IEEE Microwave and Wireless Components Letters en el 2017 [18].

Además, se analizaron métodos para controlar los valores de impedancia de las cargas propuestas. Debido a la relación no-lineal que existe entre las corrientes de control y los valores de impedancia, y dada la dificultad de obtener un modelo analítico representativo de dichas cargas, se optó por desarrollar un procedimiento de calibración basado en una técnica de aprendizaje por variedades (en inglés manifold learining). Este método utiliza datos de entrenamiento sin la necesidad de un modelo analítico previo. El método fue validado usando los prototipos de las impedancias variables y fue presentado en el trabajo Calibration of Nonlinear Variable Loads Based on Manifold Learning en la XVII reunión de trabajo en Procesamiento de la Información y Control (RPIC), en la ciudad de Mar del Plata en el 2017 [19].

Finalmente, se desarrolló un prototipo del modulador utilizando las cargas variables más simples y una antena de doble polarización. Los resultados experimentales validaron el diseño del modulador y su capacidad para controlar la polarización de la señal transmitida. Estos resultados se publicaron en el trabajo Design of a Multiport Microwave Modulator for Dynamic Polarization Reconfiguration en IEEE Transactions on Microwave Theory and Techniques en el 2019 [20].

En paralelo a la construcción del modulador, se estudiaron alternativas para el diseño de demoduladores de múltiples puertos capaces de operar con múltiples antenas. En primer lugar, se diseñó un demodulador se siete puertos capaz de operar con una antena de doble polarización. Esta arquitectura permite demodular la señal recibida y medir su polarización utilizando únicamente cuatro mediciones de potencia. Se validó la arquitectura propuesta implementando un prototipo para una frecuencia de 1575,42 MHz. Con los 
resultados preliminares de realizó el trabajo Polarimetric Demodulator Based on a Sevenport Network que fue presentado en ARGENCON 2018, en San Miguel de Tucumán en el 2018 [21].

Luego, utilizando la misma estructura de siete puertos se propuso un demodulador capaz de operar con tres antenas utilizando únicamente tres mediciones de potencia. Utilizando un prototipo se obtuvieron resultados preliminares que fueron enviados para presentar en el trabajo Design of a Seven-Port Demodulator for Wireless Applications with Multiple Antennas en la XVIII reunión de trabajo en Procesamiento de la Información y Control (RPIC), en la ciudad de Bahía Blanca en el 2019 [22]. Por último, se extendieron los conceptos desarrollados sobre el demodulador de siete puertos a $\mathrm{N}$ puertos y se utilizaron los dos ejemplos presentados para armar un trabajo de revista el cual será enviado para su publicación en IEEE Transactions on Microwave Theory and Techniques.

\subsection{Organización de la Tesis}

En el siguiente capítulo se presentan las bases teóricas sobre las redes de seis puertos y el principio de funcionamiento en aplicaciones de modulación y demodulación de señales. Asimismo, se discuten las ventajas y desventajas de estas arquitecturas frente a las diseños convencionales basadas en el uso de mezcladores.

Los Capítulos 3 a 6 contienen las contribuciones principales de esta tesis. En el Capítulo 3 se describen las bases teóricas sobre redes de siete puertos y el principio de funcionamiento de la arquitectura propuesta para la modulación de señales. Además, se presentan los diseños e implementaciones de las distintas partes que componen el modulador de siete puertos: la red de siete puertos, las impedancias variables, y la antena. Finalmente, se muestran los resultados del prototipo desarrollado que consiste en un sistema con diversidad polarimétrica.

En el Capítulo 4 se presenta el diseño de una impedancias variables desarrollada para mejoran las características del modulador presentado en el Capítulo 3.

Debido al comportamiento no lineal de las impedancias variables, uno de los problemas a resolver al momento de realizar mediciones con el prototipo del modulador consiste en definir un procedimiento de calibración adecuado. En el Capítulo 5 se presenta un método de calibración para controlar impedancias variables no-lineales. Se describe la implementación del método y su validación sobre las impedancias variables desarrolladas en el Capítulo 4.

En el Capítulo 6 se presentan las bases teóricas para el diseño de demoduladores de múltiples puertos. Se analizan dos arquitecturas basadas en una estructura de siete puertos: por un lado un demodulador de dos antenas el cual recupera las señales de dos puertos; por otro lado, un demodulador el cual permite demodular las señales recibidas por tres antenas utilizando tres detectores de potencia.

Finalmente se concluye la tesis en el Capítulo 7 donde se discuten los diseños realizados, los resultados obtenidos y se presentan las líneas de trabajo futuro. 


\section{Capítulo 2}

\section{Técnicas Seis Puertos}

En este capítulo se introducen los conceptos sobre las redes de seis puertos y su funcionamiento como modulador y demodulador de señales presentando las ventajas y desventajas de los mismos frente a los dispositivos convencionales basados en mezcladores. Finalmente se describen los modelos de señales con diversidad polarimétrica y espacial los cuales se utilizarán en capítulos posteriores.

\subsection{Antecedentes de Seis Puertos}

G. Engen y C. Hoer [23] introdujeron el concepto de seis puertos a principios de la década de 1970 como una alternativa simple y precisa de medir potencia en frecuencias de microondas. Poco después de su publicación inicial, el principio se amplió a la medición de tensión, corriente e impedancia [24], lo que dio como resultado un enfoque alternativo para el diseño de reflectómentros [25]. El principal atractivo de esta técnica de seis puertos es su capacidad de obtener información de magnitud y fase a partir de cuatro mediciones escalares de potencia o tensión. En base a este concepto, los circuitos de seis puertos han sido utilizados para una gran variedad de aplicaciones como ser analizadores de redes $[26,27]$, caracterización de materiales [28], caracterización de antenas en campo cercano [29], así como también, en sistemas de medición de polarización [30].

A mediados de la década de 1990, J. Li, R. Bosisio y K. Wu [31] presentan por primera vez el concepto de seis puertos como receptor de comunicaciones el cual se extendió rápidamente a aplicaciones de estimación de ángulo de arribo $[32,13]$. Unos años después, se propuso su aplicación para el diseño de moduladores de señales [33, 34].

\section{2. $\quad$ Red de Seis Puertos}

En aspectos generales, una estructura de seis puertos es un circuito pasivo y reciproco, compuesto por acopladores direccionales, divisores de potencia, líneas de transmisión, y desfasadores. Existen distintas topología para las redes de seis puertos las cuales dependen principalmente de la aplicación. En general, para el diseño de moduladores y demoduladores se emplean las tres estructuras mostradas en la Figura 2.1. El circuito (a) corresponde a la configuración mas utilizada que consta de un divisor Wilkinson (DW) y 


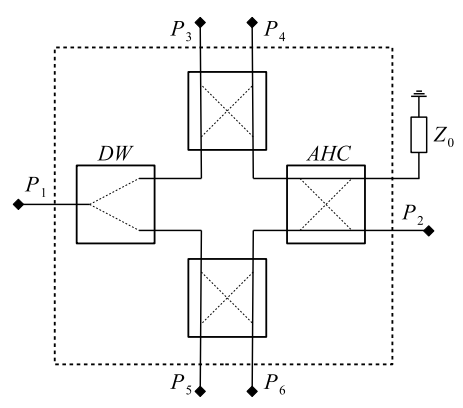

(a)

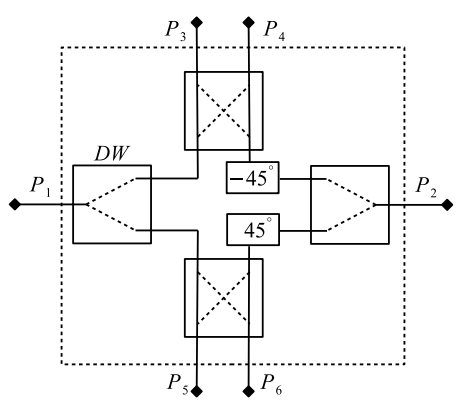

(b)

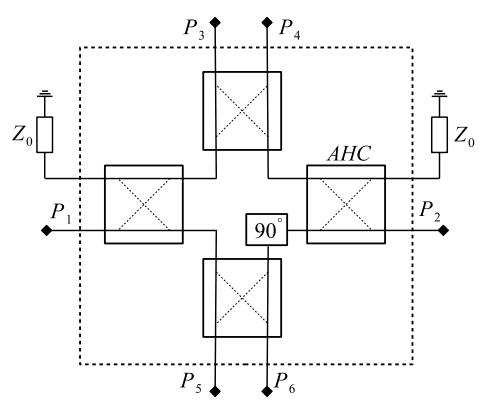

(c)

Figura 2.1: Redes de múltiples puertos utilizadas en el diseño de moduladores y demoduladores de seis puertos [1].

tres acopladores híbridos en cuadratura (AHC) [35]; el circuito (b) consta de dos DW, dos AHC y dos desfasadores de $45^{\circ}$ [36]; por último el circuito (c) se compone de la conexión entre cuatro AHC y un desfasador de $90^{\circ}$ [37].

\subsubsection{Modelo Teórico}

Las redes mencionadas son dispositivos que operan en frecuencias de microondas u ondas milimétricas. En este rango de frecuencias, los modelos clásicos de impedancia y admitancia presentan limitaciones prácticas al medir tensión y corriente debido a la dificultad de obtener cortocircuitos y circuitos abiertos estables. Por este motivo, para modelar el comportamiento de las redes se utilizan los parámetros de dispersión (scattering parameters), también conocidos como parámetros $\mathrm{S}$.

\section{Parámetros de Dispersión}

En lugar de relacionar tensiones y corrientes, los parámetros S relacionan las señales incidentes $V^{+}$y reflejadas $V^{-}$. Para explicar rápidamente este concepto se considera el sistema mostrado en la Figura 2.2. Este consiste en un generador de señales conectado a un dispositivo de un puerto (representado por la impedancia $Z_{L}$ ) a través de una líneal de transmisión de impedancia característica $Z_{0}$. Para esta configuración simple, se define como señal incidente $V^{+}$el valor de tensión $V$ medido en la entrada del dispositivo cuando la impedancia del mismo es $Z_{L}=Z_{0}$, y el valor de corriente en este caso se denota como $I=I^{+}$. Para cualquier otra condición de impedancia $Z_{L}$, la tensión $V$ y la corriente $I$ en el puerto del dispositivo se componen de la contribución de dos componentes (incidente y reflejada) como [38]

$$
\begin{aligned}
V & =V^{+}+V^{-} \\
I & =I^{+}-I^{-}
\end{aligned}
$$

donde los valores $V^{-}$e $I^{-}$son las componentes reflejadas las cuales tienen en cuenta los cambios debido a la desadaptación de impedancia $\left(Z_{L} \neq Z_{0}\right)$. Aunque los conceptos de tensión y corriente persisten en altas frecuencias, estos son remplazados por otros 


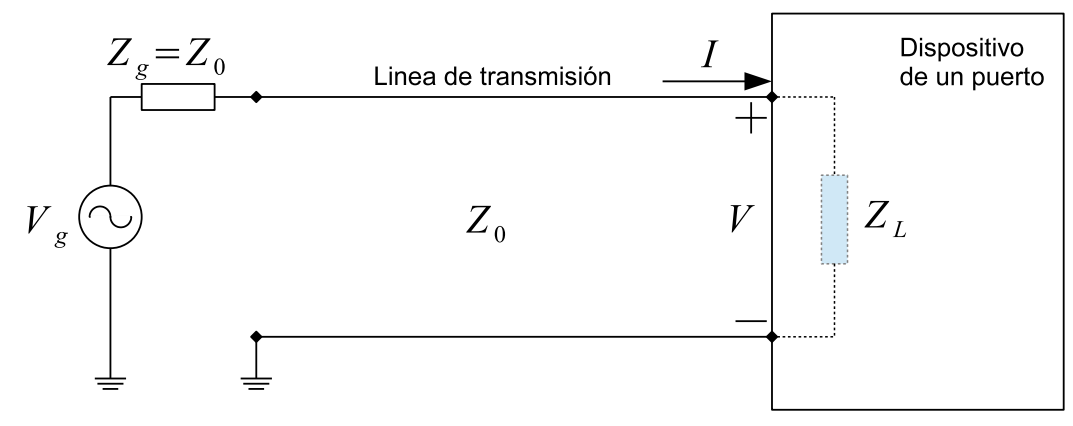

Figura 2.2: Dispositivo de un puerto conectado a una fuente de señal

parámetros para el tratamiento teórico y práctico de los circuitos. Estos parámetros son las ondas incidentes $(a)$ y reflejadas $(b)$ que se definen de la siguiente manera

$$
\begin{aligned}
a & =\frac{V^{+}}{\sqrt{Z_{0}}} \\
b & =\frac{V^{-}}{\sqrt{Z_{0}}} .
\end{aligned}
$$

La relación entre $b$ y $a$ es un valor complejo que define el parámetro de dispersión, el cual representa el comportamiento del dispositivo de un puerto, $s=\frac{b}{a}$. Además, la potencia promedio en la entrada del dispositivo se puede representar directamente por dichas componentes de la siguiente manera

$$
P=\frac{|a|^{2}-|b|^{2}}{2}
$$

Considerando un dispositivo con $N$ puertos como se muestra en la Figura 2.3, las ondas reflejadas en los $N$ puertos pueden definirse en función de los parámetros $\mathrm{S}$ y las ondas incidentes a través de la siguiente operación [39]:

$$
\left[\begin{array}{c}
b_{1} \\
\vdots \\
b_{N}
\end{array}\right]=\left[\begin{array}{ccc}
s_{11} & \cdots & s_{1 N} \\
\vdots & \ddots & \vdots \\
s_{N 1} & \cdots & s_{N N}
\end{array}\right]\left[\begin{array}{c}
a_{1} \\
\vdots \\
a_{N}
\end{array}\right]
$$

donde cada elemento de la matriz se obtiene como

$$
s_{j i}=\left.\frac{b_{j}}{a_{i}}\right|_{a_{k}=0 \operatorname{con~} \mathrm{k} \neq \mathrm{i}} .
$$

La condición $a_{k}=0$ ocurre cuando el puerto $k$ se encuentra adaptado a la impedancia característica, es decir, que se conecta a impedancias de valor $Z_{0}$. Obteniendo los valores de la matriz (2.7) se caracteriza completamente el dispositivo de $N$ puertos. A continuación se mencionan algunas propiedades de la matriz de parámetros $S$ :

- Si el dispositivo de $N$ puertos cumple la propiedad de reciprocidad, la matriz $\mathrm{S}$ es simétrica, es decir que $s_{i j}=s_{j i}$. 


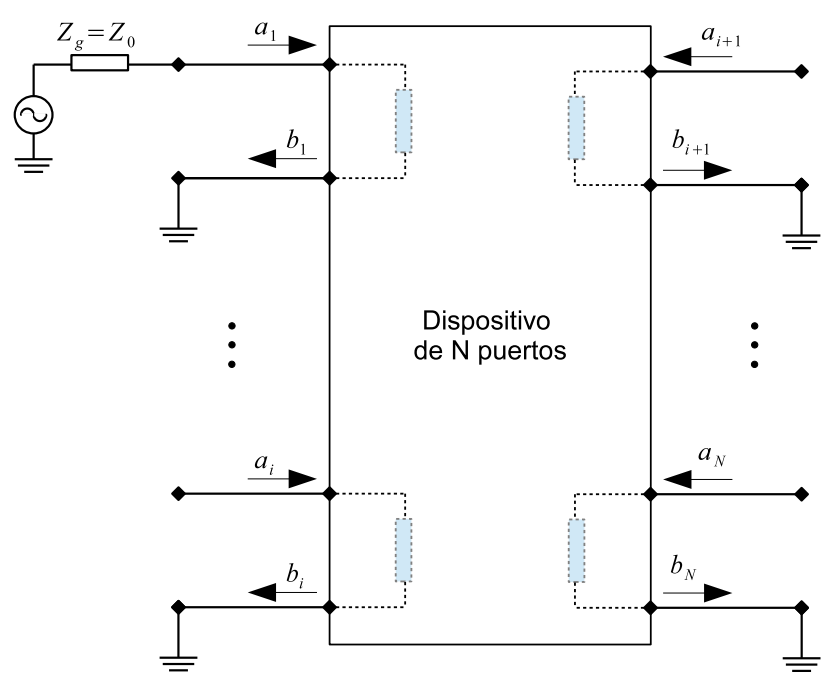

Figura 2.3: Dispositivo de $N$ puerto conectado a una fuente de señal en el puerto 1

- Si la red es pasiva y sin pérdidas, su matriz S es unitaria, es decir que su inversa es igual a la matriz hermítica.

- Si la red presenta adaptación en todos sus puertos, los elementos de la diagonal principal de la matriz son nulos.

A continuación se presentan los parámetros de las redes elementales utilizada para armar la red de seis puertos de la Figura 2.1(a).

\section{Redes Elementales}

El acoplador híbrido en cuadratura y el divisor Wilkinson se muestran en la Figura 2.4. Estas dos redes se utilizan para la construcción de una gran variedad de estructuras, entre ellas la red de seis puertos. Ambos dispositivos dividen la potencia de la señal aplicada en el puerto $P_{1}$ equitativamente entre los puertos de salida $P_{2}$ and $P_{3}$. En particular, el AHC presenta una relación de fase de $90^{\circ}$ entre sus puertos de salida, mientras que el DW mantiene una diferencia de fase de $0^{\circ}$. Siguiendo la numeración de puertos mostrada en la

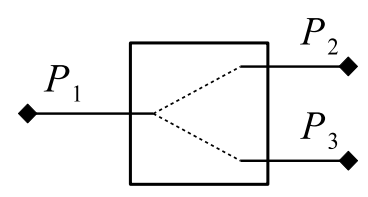

(a)

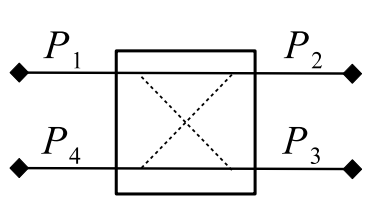

(b)

Figura 2.4: Esquema de un (a)Divisor Wilkinson y un (b) Acoplador Híbrido en cuadratura

Figura 2.4, el comportamiento ideal de estos dispositivos se representa con las siguientes 
matrices de parámetros $S$ [39]

$$
S_{W}=\frac{-1}{\sqrt{2}}\left[\begin{array}{ccc}
0 & j & j \\
j & 0 & 0 \\
j & 0 & 0
\end{array}\right], \quad S_{H}=\frac{-1}{\sqrt{2}}\left[\begin{array}{cccc}
0 & j & 1 & 0 \\
j & 0 & 0 & 1 \\
1 & 0 & 0 & j \\
0 & 1 & j & 0
\end{array}\right] .
$$

En ambas matrices se presenta la propiedad de adaptación dado que los elementos de la diagonal principal son nulos. El DW presenta aislación entre sus puertos de salida $P_{2}$ y $P_{3}$, mientras que el acoplador presenta aislación entre los puertos $P_{1}$ y $P_{4}$, así como también, entre $P_{2}$ y $P_{3}$. Las dos redes son recíprocas $\left(S_{W}=S_{W}^{T}\right.$ y $\left.S_{H}=S_{H}^{T}\right)$, el AHC es un circuito pasivo y sin pérdidas dado que $S_{H}\left(S_{H}^{T}\right)^{*}=I_{4}$ donde $I_{4}$ es una matriz identidad de dimensión $4 \times 4$, y el DW es un circuito pasivo con pérdidas.

\section{Red de Seis Puertos}

Los parámetros de la red de seis puertos mostrada en la Figura 2.1(a) se obtienen a partir de los parámetros del AHC y el DW utilizando un diagrama de grafos [2]. Este tipo de diagrama consta de un conjunto de nodos (ondas incidentes y reflejadas) y flechas (parámetros S). Cada parámetro de dispersión se representa por una flecha que comienza en el nodo correspondiente a la onda incidente y termina en el nodo correspondiente a la onda reflejada. Si no hay conexión entre los nodos el parámetro es nulo. Por lo tanto, debido a las características de aislación y adaptación de las redes elementales, el diagrama de grafos de la red de seis puertos resulta muy simple. Por ejemplo, al aplicar una señal en el puerto $P_{1}$ se obtiene el diagrama mostrado en la Figura 2.5. Notar que, la conexión

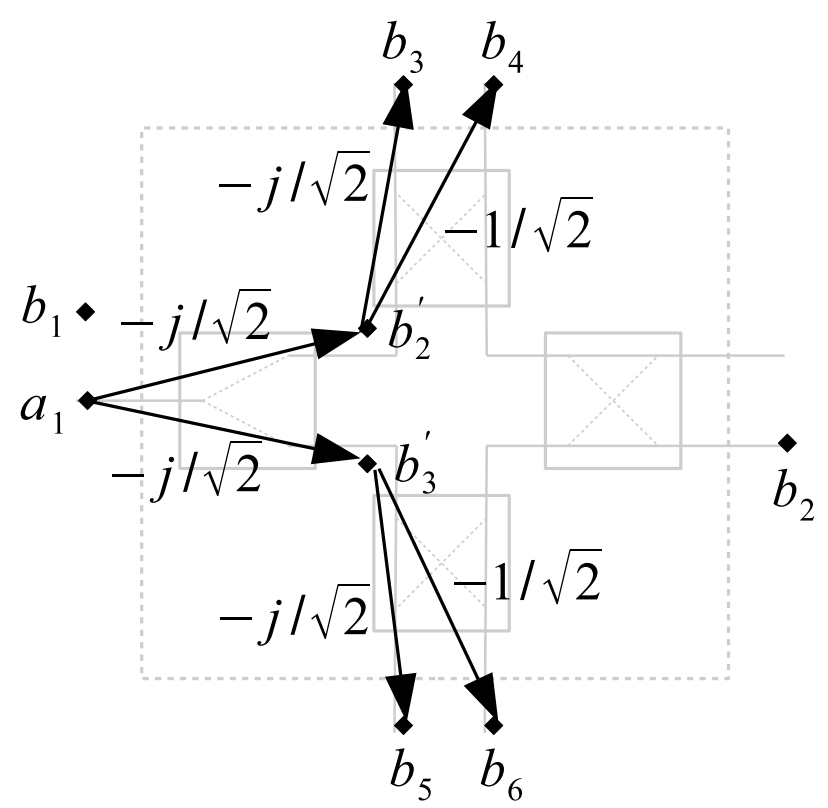

Figura 2.5: Diagrama de grafo establecido cuando se excita el puerto $P_{1}$

entre los puertos del DW y el AHC se representan por los nodos $b_{2}^{\prime}$ y $b_{3}^{\prime}$ que corresponden a las ondas emergentes a la salida del divisor. Por lo tanto, se calcula el parámetros que 
relaciona la onda reflejada en el puerto $P_{3}$ con la onda incidente en $P_{1}$ aplicando la teoría de grafos para dos ramas en serie mostrada en la Figura 2.6. Los restantes parámetros

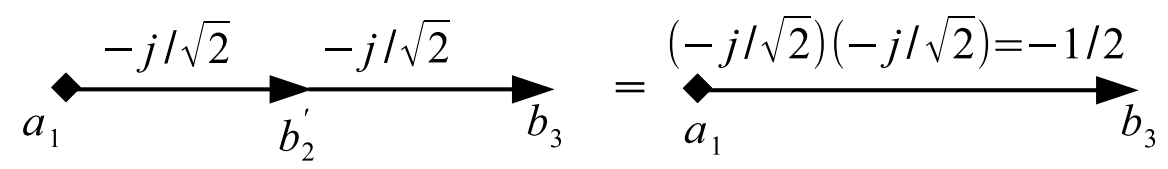

Figura 2.6: Teoría de grafos, ramas en serie [2]

asociados con la entrada $a_{1}$ se obtienen de la misma manera. Notar que, no hay ninguna conexión con los nodos $b_{1}$ y $b_{2}$ por lo tanto los parámetros son nulos $s_{11}=s_{21}=0$. Para obtener los parámetros asociados a otra entrada se aplica el mismo procedimiento excitando la entrada correspondiente. Finalmente, siguiendo la estrategia mencionada, la matriz de parámetros $\mathrm{S}$ de la red de seis puertos resulta

$$
S=\frac{1}{2}\left[\begin{array}{cccccc}
0 & 0 & -1 & j & -1 & j \\
0 & 0 & j & -1 & 1 & j \\
-1 & 1 & 0 & 0 & 0 & 0 \\
j & j & 0 & 0 & 0 & 0 \\
-1 & j & 0 & 0 & 0 & 0 \\
j & -1 & 0 & 0 & 0 & 0
\end{array}\right]
$$

Para simplificar la notación se definen los parámetros marcados en color rojo como

$$
S_{r}=\frac{1}{2}\left[\begin{array}{cccc}
-1 & j & -1 & j \\
1 & j & j & -1
\end{array}\right] .
$$

En las siguientes secciones se describe el funcionamiento del demodulador y el modulador basados en esta estructura de seis puertos.

\subsection{Demodulador de Seis Puertos}

El esquema básico del demodulador de seis puertos se muestra en la Figura 2.7. Este opera de la siguiente manera: una señal de oscilador local se aplica en el puerto $P_{1}$, mientras que la señal recibida por la antena se amplifica y se inyecta en el puerto $P_{2}$. Dada la relación entre los puertos de las red estas dos señales se combinan con diferentes fases en los puertos de salida $P_{3}$ a $P_{6}$. Entonces, debido a la operación no lineal de los detectores de potencia se generar la conversión de frecuencia. Finalmente en cada salida se conecta un filtro pasabajos que elimina las componentes de alta frecuencia obteniendo la señal deseada en banda base.

\section{Modelo Ideal}

Siguiendo el modelo de parámetros $\mathrm{S}$ de la red de seis puertos (2.10) se obtiene la relación entre las señales de salida en los puertos $P_{3}$ a $P_{6}$ y las señales de entrada en los 


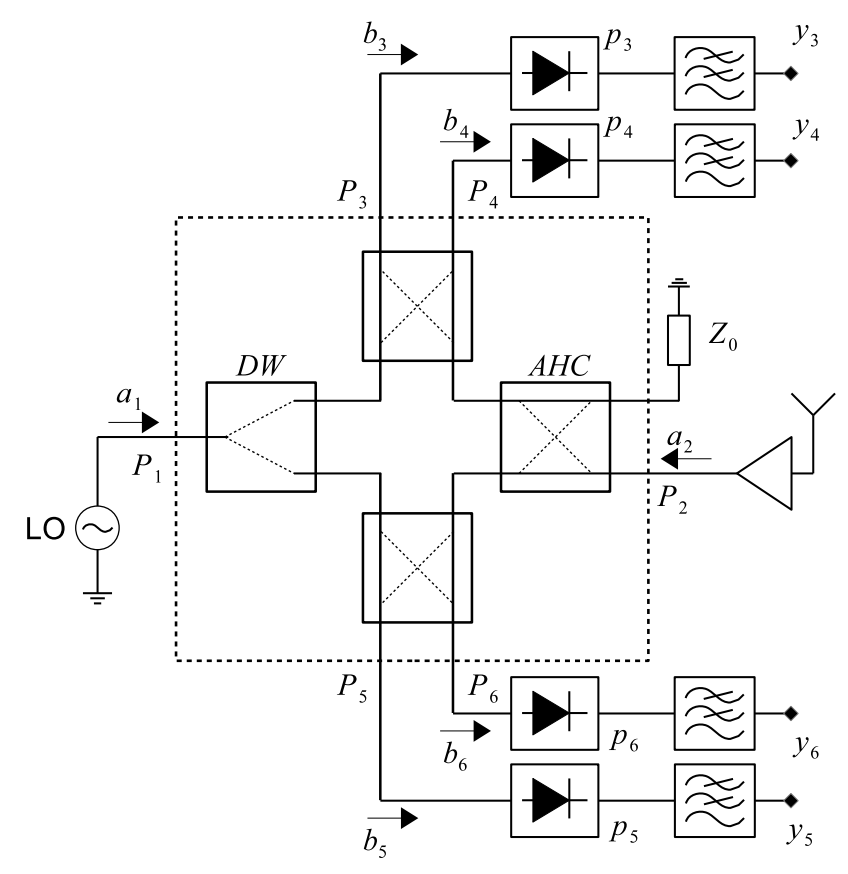

Figura 2.7: Demodulador basado en una red de seis puertos.

puertos $P_{1}$ y $P_{2}$ de la red

$$
\left[\begin{array}{l}
b_{3} \\
b_{4} \\
b_{5} \\
b_{6}
\end{array}\right]=\frac{1}{2}\left[\begin{array}{cc}
-1 & 1 \\
j & j \\
-1 & j \\
j & -1
\end{array}\right]\left[\begin{array}{l}
a_{1} \\
a_{2}
\end{array}\right]
$$

Notar que a partir de (2.12) dos observaciones $\left(\begin{array}{lll}b_{3} & \text { y } & b_{4}\end{array}\right)$ son suficientes para obtener las amplitudes complejas de $a_{1}$ y $a_{2}$. Sin embargo, en la práctica medir amplitudes complejas no es una tarea sencilla en frecuencias de microondas. Por el contrario, se utilizan mediciones escalares de potencia. Considerando que los instrumentos de medición están perfectamente adaptados a los puertos de salida, entonces la potencia promedio resulta

$$
\begin{gathered}
y_{3}=\frac{\left|b_{3}\right|^{2}}{2}=\frac{1}{8}\left|-a_{1}+a_{2}\right|^{2} \\
y_{4}=\frac{\left|b_{4}\right|^{2}}{2}=\frac{1}{8}\left|j a_{1}+j a_{2}\right|^{2} \\
y_{5}=\frac{\left|b_{5}\right|^{2}}{2}=\frac{1}{8}\left|-a_{1}+j a_{2}\right|^{2} \\
y_{6}=\frac{\left|b_{6}\right|^{2}}{2}=\frac{1}{8}\left|j a_{1}-a_{2}\right|^{2}
\end{gathered}
$$

y desarrollando el módulo cuadrado se obtiene

$$
\begin{array}{r}
y_{3}=\frac{\left|a_{1}\right|^{2}}{8}+\frac{\left|a_{2}\right|^{2}}{8}+\frac{1}{4} \Re\left\{-a_{1} a_{2}^{*}\right\} \\
y_{4}=\frac{\left|a_{1}\right|^{2}}{8}+\frac{\left|a_{2}\right|^{2}}{8}+\frac{1}{4} \Re\left\{a_{1} a_{2}^{*}\right\}
\end{array}
$$




$$
\begin{array}{r}
y_{5}=\frac{\left|a_{1}\right|^{2}}{8}+\frac{\left|a_{2}\right|^{2}}{8}+\frac{1}{4} \Re\left\{j a_{1} a_{2}^{*}\right\} \\
y_{6}=\frac{\left|a_{1}\right|^{2}}{8}+\frac{\left|a_{2}\right|^{2}}{8}+\frac{1}{4} \Re\left\{-j a_{1} a_{2}^{*}\right\} .
\end{array}
$$

Debido a la simetría de la estructura de seis puertos, hay componentes comunes entre los valores de salida $y_{3}-y_{6}$. En consecuencia, la componente se obtiene la amplitud compleja de la señal de interés realizando la siguiente combinación lineal de las salidas [40]

$$
\begin{aligned}
& \omega_{1}=y_{4}-y_{3}=\frac{1}{2} \Re\left\{a_{1} a_{2}^{*}\right\} \\
& \omega_{2}=y_{6}-y_{5}=\frac{1}{2} \Im\left\{a_{1} a_{2}^{*}\right\}
\end{aligned}
$$

El valor de $a_{1}$ define la amplitud compleja del oscilador local, por lo tanto asumiendo que su valor es conocido se obtiene

$$
a_{2}=\frac{2}{a_{1}^{*}}\left(\omega_{1}-j \omega_{2}\right)
$$

Hasta este punto se considera que las amplitudes complejas de las señales de entrada son constantes. Sin embargo, un demodulador recibe señales cuya envolvente varia en el tiempo. Denotando $x_{B P}(t)$ a la señal pasabanda de entrada en el puerto $P_{2}$, esta señal tiene una frecuencia central de $f_{c}$ y se puede escribir en su componente en fase y cuadratura de la siguiente forma [41]

$$
x_{B P}(t)=x_{I}(t) \cos \left(2 \pi f_{c} t\right)-x_{Q}(t) \sin \left(2 \pi f_{c} t\right)
$$

donde $x(t)=x_{I}(t)+j x_{Q}(t)$ es la envolvente compleja y $x_{I}(t)$ y $x_{Q}(t)$ son sus componentes en fase y cuadratura. Otra forma de escribir la misma señal es

$$
x_{B P}(t)=\Re\left\{x(t) e^{j 2 \pi f_{c} t}\right\} .
$$

Cuando una señal se inyecta en la entrada $P_{2}$ y alcanza las salidas $P_{i}$ con $i \in\{3,4,5,6\}$, sufre un cambio de amplitud y fase definido por los parámetros $\mathrm{S}$

$$
x_{B P}^{(i)}(t)=\Re\left\{x(t) e^{j 2 \pi f_{c} t}\left|s_{i 2}\right| e^{j L s_{i 2}}\right\}
$$

donde el índice $i$ denota el número del respectivo puerto de salida. Asimismo, la señal del OL la cual se inyecta en el puerto $P_{1}$ se define como $x_{O L}(t)=G \cos \left(2 \pi f_{o} t+\phi_{o}\right)$, o equivalente $x_{O L}(t)=\Re\left\{G e^{j \phi_{o}} e^{j 2 \pi f_{o} t}\right\}$, y su expresión en el puerto de salida $P_{i}$ es

$$
x_{O L}^{(i)}(t)=\Re\left\{G e^{j \phi_{o}} e^{j 2 \pi f_{o} t}\left|s_{i 1}\right| e^{j L s_{i 1}}\right\} .
$$

\section{Conversión de Frecuencia}

La conversión de frecuencia en el demodulador de seis puertos se realiza por mezcla aditiva de (2.26) y (2.27), seguida de una operación no lineal. En particular los detectores de potencia se basan en diodos Schottky operando en la zona cuadrática cuya salida se puede representar por la serie de Taylor como

$$
p=K_{0}+K_{1} q+K_{2} q^{2}+\ldots
$$


remplazando $q$ por la suma de las señales de entrada en el puerto de salida $P_{i}$ se obtiene

$$
p_{i}(t) \approx K_{0}+K_{1}\left(x_{B P}^{(i)}(t)+x_{O L}^{(i)}(t)\right)+K_{2}\left(x_{B P}^{(i)}(t)+x_{O L}^{(i)}(t)\right)^{2} .
$$

Notar que, en (2.29) se han despreciado los términos de orden superior. Al aplicar el filtro pasabajos en la salida de cada detector se eliminan las componentes de alta frecuencia de la expresión anterior. Entre ellos, el término afectado por la constante $K_{1}$ que consta de señales en frecuencia $f_{r}$ y $f_{c}$. Asimismo, el término cuadrático genera componentes en frecuencias $2 f_{r}, 2 f_{c}$ y $f_{r}+f_{c}$ que también serán eliminadas por el filtro pasabajos. Por lo tanto, la expresión a la salida del filtro resulta [41]

$$
\begin{aligned}
y_{i}(t)= & K_{0}+\frac{K_{2}}{2}\left(\left|s_{i 2}\right|^{2} x_{I}^{2}(t)+\left|s_{i 2}\right|^{2} x_{Q}^{2}(t)+\left|s_{i 1}\right|^{2} G^{2}\right) \\
& +K_{2}\left|s_{i 2}\right|\left|s_{i 1}\right| x_{I}(t) G \cos \left(2 \pi\left(f_{0}-f_{c}\right) t+\angle s_{i 1}-\angle s_{i 2}\right) \\
& \left.+K_{2}\left|s_{i 2}\right|\left|s_{i 1}\right| x_{Q}(t) G \sin \left(2 \pi\left(f_{0}-f_{c}\right) t+\angle s_{i 1}-\angle s_{i 2}\right)\right) .
\end{aligned}
$$

Si el oscilador local y la señal $x_{B P}(t)$ tienen la misma frecuencia $f_{0}=f_{c}$ la conversión de frecuencia a banda base es directa (receptor homodino), entonces la ecuación (2.30) se reduce a

$$
\begin{aligned}
y_{i}(t)= & \frac{K_{2}}{2}\left(\left|s_{i 2}\right|^{2} x_{I}^{2}(t)+\left|s_{i 2}\right|^{2} x_{Q}^{2}(t)+\left|s_{i 1}\right|^{2} G^{2}\right) \\
& +K_{2}\left|s_{i 2}\right|\left|s_{i 1}\right|\left(x_{I}(t) G \cos \left(\phi_{i}\right)+x_{Q}(t) G \sin \left(\phi_{i}\right)\right)
\end{aligned}
$$

donde $\phi_{i}=\angle s_{i 1}-\angle s_{i 2}$.

\section{Expresiones de Demodulación}

Agrupando las cuatro mediciones de potencia y despejando el factor $K_{2} G$ se obtiene $\frac{2}{K_{2} G}\left[\begin{array}{c}y_{3}(t) \\ y_{4}(t) \\ y_{5}(t) \\ y_{6}(t)\end{array}\right]=\left[\begin{array}{lll}2\left|s_{32}\right|\left|s_{31}\right| \cos \left(\phi_{3}\right) & 2\left|s_{32}\right|\left|s_{31}\right| \sin \left(\phi_{3}\right) & \left|s_{32}\right|^{2} \\ 2\left|s_{42}\right|\left|s_{41}\right| \cos \left(\phi_{4}\right) & 2\left|s_{42}\right|\left|s_{41}\right| \sin \left(\phi_{4}\right) & \left|s_{42}\right|^{2} \\ 2\left|s_{52}\right|\left|s_{51}\right| \cos \left(\phi_{5}\right) & 2\left|s_{52}\right|\left|s_{51}\right| \sin \left(\phi_{5}\right) & \left|s_{52}\right|^{2} \\ 2\left|s_{62}\right|\left|s_{61}\right| \cos \left(\phi_{6}\right) & 2\left|s_{62}\right|\left|s_{61}\right| \sin \left(\phi_{6}\right) & \left|s_{62}\right|^{2}\end{array}\right]\left[\begin{array}{c}x_{I}(t) \\ x_{Q}(t) \\ x_{I}^{2}(t)+x_{Q}^{2}(t)\end{array}\right]+\left[\begin{array}{c}\left|s_{31}\right|^{2} \\ \left|s_{41}\right|^{2} \\ \left|s_{51}\right|^{2} \\ \left|s_{61}\right|^{2}\end{array}\right]$.

$\mathrm{Al}$ remplazar los parámetros $\mathrm{S}$ por los valores (2.10) resulta

$$
\frac{2}{K_{2} G}\left[\begin{array}{l}
y_{3}(t) \\
y_{4}(t) \\
y_{5}(t) \\
y_{6}(t)
\end{array}\right]=\frac{1}{4}\left[\begin{array}{ccc}
-2 & 0 & 1 \\
2 & 0 & 1 \\
0 & -2 & 1 \\
0 & 2 & 1
\end{array}\right]\left[\begin{array}{c}
x_{I}(t) \\
x_{Q}(t) \\
x_{I}^{2}(t)+x_{Q}^{2}(t)
\end{array}\right]+\frac{1}{4}\left[\begin{array}{l}
1 \\
1 \\
1 \\
1
\end{array}\right]
$$

Finalmente, realizando la operación (2.21) y (2.22) se obtiene las componentes en fase y en cuadratura de la envolvente compleja como

$$
\left[\begin{array}{c}
x_{I}(t) \\
x_{Q}(t)
\end{array}\right]=\frac{2}{K_{2} G}\left[\begin{array}{c}
\omega_{1}(t) \\
\omega_{2}(t)
\end{array}\right] .
$$

La relación directa entre las señales $\omega_{1}$ y $\omega_{2}$ con las componentes de la envolvente compleja ocurre en condiciones ideales, cuando la red se modela como (2.10). En la práctica, los pequeños desbalances en el comportamiento de la red dificultan la recuperación precisa de la envolvente. Por lo tanto, se utiliza la expresión (2.32) y un procedimiento de calibración para medir las constantes de la matriz [38]. 


\section{Comparación}

Los receptores basados en redes de seis puertos son un tipo especial de receptores de conversión directa. Este tipo de arquitecturas no remplaza a los dispositivos convencionales sin embargo, en aplicaciones donde se requieren pequeños tamaños y bajo consumo pueden ser la alternativa mas conveniente.

Una desventaja importante de la red de seis puertos es su baja sensibilidad y su nivel limitado de ruido (propios de los receptores homodinos), debido a esto, es recomendable el uso de un amplificador de bajo ruido (en inglés LNA) en el puerto de entrada. El esfuerzo adicional de utilizar cuatro conversores analógico-digitales (ADC), provoca un aumento en el número de componentes del circuito y en el consumo de energía. Esto es aceptable y recomendable porque permite realizar una calibración completa del receptor compensando las imperfecciones que existen en la red. El gran ancho de banda que poseen las redes de seis puertos, es consecuencia directa de la utilización de acopladores direccionales y divisores de potencia para formar la estructura, éstos tienen un ancho de banda de al menos $10 \%$ de su frecuencia de diseño. A modo de resumen, el Cuadro 2.1 presenta las ventajas y desventajas de los receptores de seis puertos frente a las arquitecturas heterodinas convencionales.

\begin{tabular}{ll}
\hline \hline Ventajas & Desventajas \\
\hline Gran ancho de banda & Baja sensibilidad \\
Diseño escalable con la frecuencia & Alta figura de ruido \\
Baja complejidad circuital & El doble de conversores A/D \\
Completamente pasivo & \\
Front-end calibrables & \\
Versatilidad para distintas frecuencias y aplicaciones & \\
\hline
\end{tabular}

Cuadro 2.1: Tabla de ventajas y desventajas del demodulador de seis puertos Vs demoduladores convencionales.

\subsection{Modulador de Seis Puertos}

El esquema básico de un modulador de seis puertos se muestra en la Figura 2.8. Al aplicar una señal de OL en el puerto $P_{1}$, esta se propaga dentro de la estructura de seis puertos hacia los puertos $P_{3}$ a $P_{6}$. Las señales de salida en dichos puertos se reflejan en las impedancias de carga $Z_{3}$ a $Z_{6}$ retornando a la estructura de seis puertos. Una parte de las señales que inciden el los puertos $P_{3}$ a $P_{6}$ se transfiere al puerto de salida $P_{2}$. Dependiendo del valor seleccionado de las impedancias de carga $Z_{3}-Z_{6}$, se establece la amplitud compleja de la señal de salida $b_{2}$. La modulación se logra al variar los valores de las impedancias con señales de banda base. Este tipo de modulador corresponde a una arquitectura de conversión directa y suele llamarse modulador de portadora directa o en ingles, direct-carrier modulator. 


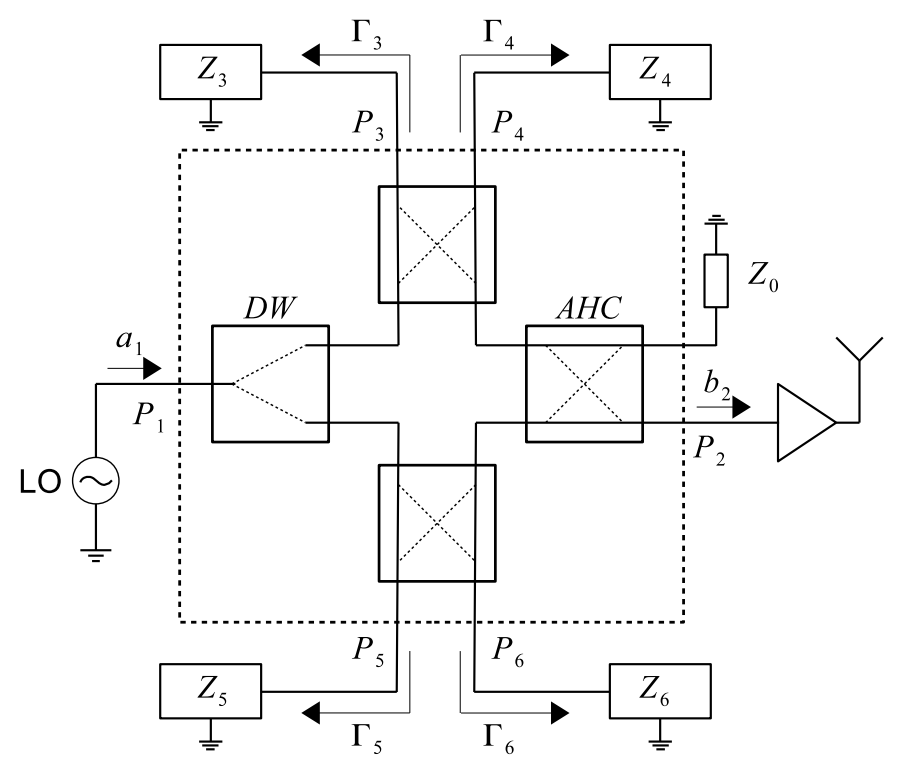

Figura 2.8: Modulador basado en una red de seis puertos.

\section{Modelo Teórico}

Para modelar la relación entre los distintos puertos de la red de seis puertos se utiliza la matriz (2.10), la cual sigue la misma numeración presentada en la Figura 2.8. El puerto $P_{2}$ es la salida, y $P_{1}$ la entrada donde se aplica la señal de OL. Los restantes puertos se conectan a las impedancias variables y se denotan como $P_{i}$ donde $i$ toma los valores $\{3,4,5,6\}$. La relación de magnitud y fase entre la onda incidente y reflejada en cada puerto $P_{i}$ esta definida por la impedancia $z_{i}$ como sigue

$$
a_{i}=\Gamma_{i} b_{i}
$$

donde $\Gamma_{i}$ es el coeficiente de reflexión debido a la impedancia $Z_{i}$. Dicho coeficiente mide la desadaptación que se produce entre la impedancia conectada y la impedancia característica $Z_{0}$ y se define como

$$
\Gamma_{i}=\frac{Z_{i}-Z_{0}}{Z_{i}+Z_{0}}
$$

En particular, se asume que la impedancia característica de todos los puertos es la misma y vale $Z_{0}$. Dado que hay cuatro impedancias de carga, la expresión (2.35) se puede agrupar en una matriz

$$
\left[\begin{array}{l}
a_{3} \\
a_{4} \\
a_{5} \\
a_{6}
\end{array}\right]=\Gamma_{r}\left[\begin{array}{l}
b_{3} \\
b_{4} \\
b_{5} \\
b_{6}
\end{array}\right]
$$


donde $\Gamma_{r}$ es una matriz diagonal cuyos elementos son los coeficientes de reflexión que presentan las cargas. Luego utilizando la matriz ideal (2.10) se obtiene

$$
\left[\begin{array}{l}
b_{1} \\
b_{2} \\
b_{3} \\
b_{4} \\
b_{5} \\
b_{6}
\end{array}\right]=\left[\begin{array}{cc}
0 & S_{r} \\
S_{r}^{T} & 0
\end{array}\right]\left[\begin{array}{cc}
I_{2} & 0 \\
0 & \Gamma_{r}
\end{array}\right]\left[\begin{array}{l}
a_{1} \\
a_{2} \\
b_{3} \\
b_{4} \\
b_{5} \\
b_{6}
\end{array}\right] .
$$

donde $I_{2}$ es una matriz identidad de dimensión $2 \times 2$. La expresión anterior se puede separar en dos términos

$$
\begin{aligned}
& {\left[\begin{array}{l}
b_{1} \\
b_{2}
\end{array}\right]=S_{r} \Gamma_{r}\left[\begin{array}{l}
b_{3} \\
b_{4} \\
b_{5} \\
b_{6}
\end{array}\right]} \\
& {\left[\begin{array}{l}
b_{3} \\
b_{4} \\
b_{5} \\
b_{6}
\end{array}\right]=S_{r}^{T}\left[\begin{array}{l}
a_{1} \\
a_{2}
\end{array}\right]}
\end{aligned}
$$

Entonces, remplazando (2.40) en (2.39), se obtiene

$$
\left[\begin{array}{l}
b_{1} \\
b_{2}
\end{array}\right]=S_{r} \Gamma_{r} S_{r}^{T}\left[\begin{array}{l}
a_{1} \\
a_{2}
\end{array}\right] .
$$

Asumiendo que los dispositivos conectados al puerto de salida $P_{2}$ están perfectamente adaptados a la impedancia característica, entonces $a_{2}=0$. Entonces, considerando los parámetros ideales en (2.11) la señal de salida resulta

$$
b_{2}=-\frac{a_{1}}{4}\left[\left(\Gamma_{3}+\Gamma_{4}\right)+j\left(\Gamma_{5}+\Gamma_{6}\right)\right] .
$$

Normalmente, se suelen definir los factores $\Gamma_{I}=\Gamma_{3}+\Gamma_{4}$ en referencia a la componente en fase de la señal de salida, y $\Gamma_{Q}=\Gamma_{5}+\Gamma_{6}$ en referencia a la componente en cuadratura. En estas condiciones la expresión anterior se reduce a

$$
b_{2}=-\frac{a_{1}}{4}\left[\Gamma_{I}+j \Gamma_{Q}\right] .
$$

La mayoría de los moduladores de seis puertos utilizan impedancias variables de valor real. Como consecuencia, el factor $\Gamma_{I}$ establece la componente en fase de la señal de salida mientras que $\Gamma_{Q}$ establece la componente en cuadratura. En general, los moduladores de seis puertos se proponen para implementar modulaciones digitales, entre ellas M-PSK y M-QAM [42]-[47].

\section{Comparación}

La utilización de impedancias variables en lugar de mezcladores presenta una de las ventajas principales de los moduladores de seis puertos debido a la gran diferencia de costo y consumo de energía. Una característica importante de los moduladores de seis puertos es 
la estrecha relación entre la potencia de la señal modulada y la potencia del OL, como se ilustra en (2.43). Esta relación proporciona la capacidad de controlar directamente el nivel de la señal de salida a través de la potencia de entrada evitando el uso de amplificadores de ganancia variable los cuales reducen el ancho de banda del sistema. Esta característica resulta de mucho interés en aplicaciones de corto alcance que requieren altos anchos de banda y baja potencia de transmisión. Por el contrario, los moduladores convencionales basados en mezcladores, como la celda Gilbert, requieren un cierto nivel de potencia de OL para funcionar adecuadamente y presentan anchos de banda muy estrechos en frecuencias de microondas. Los moduladores de seis consisten en esquemas de conversión directa (homodino), lo cuales presentan problemas de fuga de la señal de OL consecuencia de la baja aislación y filtrado entre la entrada de OL y la salida del modulador. Sin embargo, gracias al procesamiento analógico de señales mediante técnicas sencillas de microondas $[44,46]$, estos problemas se reducen enormemente en los moduladores de seis puertos. En el Cuadro 2.2 se presentan las ventajas y desventajas de los moduladores de seis puertos.

\begin{tabular}{ll}
\hline \hline Ventajas & Desventajas \\
\hline Gran ancho de banda & Cuatro señales de banda base \\
Diseño escalable con la frecuencia & \\
Baja complejidad circuital & \\
Completamente pasivo & \\
Front-end calibrables & \\
Bajo nivel de oscilador local & \\
\hline
\end{tabular}

Cuadro 2.2: Tabla de ventajas y desventajas del modulador de seis puertos.

\subsection{Esquema con Múltiples Antenas}

Como se mencionó en el Capítulo 1, el uso de más de una antena permite transmitir y recibir señales electromagnéticas con distinta polarización, así como también, conformar el haz de transmisión y detectar el ángulo de arribo de fuentes de señal en el receptor. A continuación, se presentan las expresiones que consideran las características de la polarización y del ángulo de arribo de las señales en un esquema con dos antenas, el cual se utiliza en los dispositivos desarrollados en esta tesis.

\subsubsection{Representación de la Polarización}

La polarización de una onda electromagnética representa la orientación del campo. Específicamente, la polarización del campo eléctrico es una elipse que describe el extremo de dicho vector en el plano ortogonal a la dirección de propagación de la señal al transcurrir el tiempo. Considerando una onda plana propagándose en la dirección $\hat{z}$ (versor en la dirección del vector z.), el campo eléctrico instantáneo es

$$
\tilde{\mathbf{E}}=\left[\begin{array}{c}
\tilde{E}_{x} \\
\tilde{E}_{y}
\end{array}\right]=\left[\begin{array}{c}
\left|E_{0 x}\right| \sin \left(\omega t-k z+\delta_{x}\right) \\
\left|E_{0 y}\right| \sin \left(\omega t-k z+\delta_{y}\right)
\end{array}\right]
$$

donde los fasores $E_{x}=\left|E_{0 x}\right| e^{j \delta_{x}}$ y $E_{y}=\left|E_{0 y}\right| e^{j \delta_{y}}$ definen la amplitud y fase de las componentes del campo en las direcciones $\hat{x}$ e $\hat{y}$, respectivamente. La elipse de polarización 
de dicha onda se puede representar en función del ángulo de orientación $\alpha$ y del ángulo de elipticidad $\beta$, como se muestra en la Fig. 2.9. Los ángulos $\alpha$ y $\beta$ se relacionan con las componentes del vector campo eléctrico de la siguiente manera

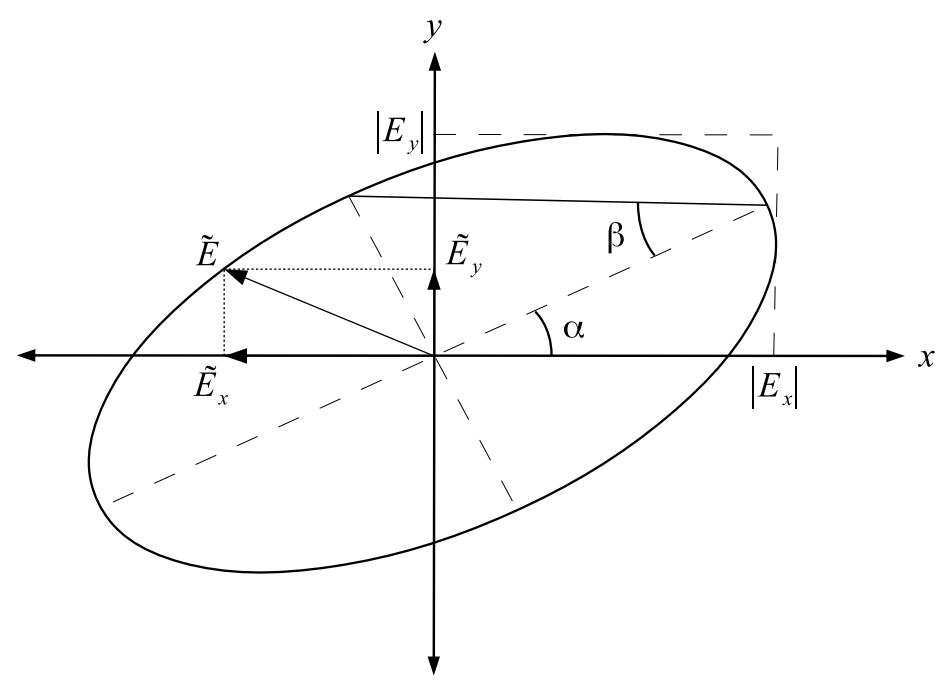

Figura 2.9: Elipse de polarización

$$
\left[\begin{array}{l}
E_{x} \\
E_{y}
\end{array}\right]=E_{0}\left[\begin{array}{cc}
\cos \alpha & -\sin \alpha \\
\sin \alpha & \cos \alpha
\end{array}\right]\left[\begin{array}{c}
\cos \beta \\
j \sin \beta
\end{array}\right]
$$

donde $\alpha \in\left(-90^{\circ}, 90^{\circ}\right], \beta \in\left[-45^{\circ}, 45^{\circ}\right]$ y el fasor $E_{0}=\sqrt{\left|E_{x}\right|^{2}+\left|E_{y}\right|^{2}} e^{j \delta_{x}}$ define la amplitud y fase absoluta del campo eléctrico. Por otro lado, se obtienen los ángulos $\alpha$ y $\beta$ a partir de las siguientes expresiones desarrolladas en [48]

$$
\begin{aligned}
& \tan 2 \alpha=2 \cos \left(\delta_{y}-\delta_{x}\right) \frac{\left|E_{x}\right|\left|E_{y}\right|}{\left|E_{x}\right|^{2}-\left|E_{y}\right|^{2}} \\
& \sin 2 \beta=2 \sin \left(\delta_{y}-\delta_{x}\right) \frac{\left|E_{x}\right|\left|E_{y}\right|}{\left|E_{x}\right|^{2}+\left|E_{y}\right|^{2}} .
\end{aligned}
$$

Un caso particular es la polarización lineal, que ocurre cuando $\delta_{x}=\delta_{y}(\beta=0)$. Otro caso importante es la polarización circular, que se produce cuando $\left|E_{x}\right|=\left|E_{y}\right|$ y $\delta_{y}-\delta_{x}= \pm 90^{\circ}$ $\left(\beta= \pm 45^{\circ}\right)$.

Finalmente, una onda plana de polarización arbitraria se puede componer como la combinación lineal de dos ondas de polarización lineal ortogonales. De esta forma, (2.45) muestra la relación de amplitud y fase entre componentes de polarización lineal en las direcciones $\hat{x}$ e $\hat{y}$ para generar una onda con ángulos de polarización $\alpha$ y $\beta$.

\subsubsection{Representación del Ángulo de Arribo}

En la Figura 2.10 se presenta un esquema con antenas receptoras separadas una distancia $d$. Debido al ángulo de incidencia $\theta$ de la señal recibida, existe una diferencia de 


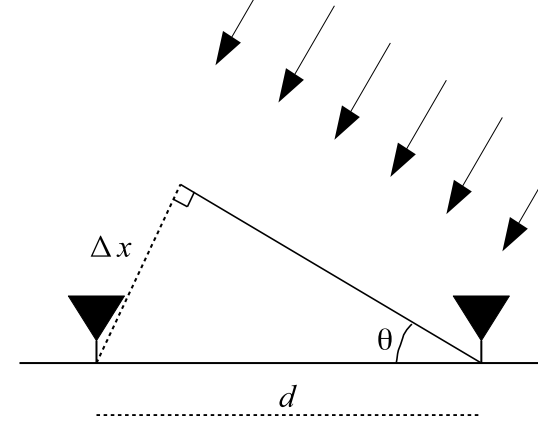

Figura 2.10: Modelo geométrico

distancia $(\Delta x)$ entre los dos caminos de propagación de la señal. Como consecuencia, la señal en una de las antenas presenta una diferencia de fase de $\Delta \phi$. Esta diferencia de fase está directamente relacionada con la distancia $\Delta x$ como

$$
\Delta x=\lambda \frac{\Delta \phi}{2 \pi}
$$

por lo tanto, el ángulo de incidencia está dado por

$$
\sin \theta=\frac{\Delta x}{d}=\frac{\lambda}{d} \frac{\Delta \phi}{2 \pi}
$$

En este marco, las amplitudes complejas de las señales recibidas por las dos antenas se modelan de la siguiente manera

$$
\left[\begin{array}{c}
S_{a 1} \\
S_{a 2}
\end{array}\right]=S_{0}\left[\begin{array}{c}
1 \\
e^{j \Delta \phi}
\end{array}\right]
$$

donde $S_{0}$ es la amplitud compleja absoluta de la señal recibida. Un problema clásico de estimación del ángulo de arribo consiste en calcular el valor de $\theta$ a partir de la combinación de las señales recibidas. Por otro lado, usando el teorema de reciprocidad, al transmitir la señal (2.50) con dos antenas separadas una distancia $d$ se puede orientar el haz del arreglo en la dirección de $\theta$. 


\section{Capítulo 3}

\section{Modulador de Siete Puertos}

En este capítulo se presentan los fundamentos teóricos, el diseño y la implementación de un sistema de modulación de la polarización basado en una red de siete puertos. Se demuestra que es posible generar señales de polarización arbitraria y modular la envolvente compleja de la señal transmitida, seleccionando adecuadamente los coeficientes de reflexión de cuatro cargas variables conectadas en los puertos de carga de la red. La arquitectura propuesta opera en forma similar a los moduladores de seis puertos, con la ventaja adicional de contar con dos salidas en lugar de una. A partir del modelo teórico, se analizan las características de eficiencia de potencia y pérdidas por retorno del sistema.

\subsection{Descripción del Dispositivo}

La Figura 3.1 muestra el esquema básico del sistema de modulación propuesto. La estructura de siete puertos consta de un puerto de entrada $P_{1}$ el cual se conecta al oscilador local, y dos puertos de salida $P_{2}$ y $P_{3}$ que alimentan dos antenas o una misma antena de dos puertos. Los restantes cuatro puertos, $P_{4}$ a $P_{7}$, contienen cargas variables que deben ser diseñadas para direccionar la energía de la señal de OL a los puertos de salida con el balance de amplitud y la diferencia de fase adecuados para obtener las señales de salida $b_{2}$ y $b_{3}$ deseadas. Por otro lado, el circuito de banda base provee las señales correspondientes para controlar los valores de impedancia de las cargas en los puertos $P_{4}$ a $P_{7}$. La velocidad de cambio de las cargas variables determina el ancho de banda de la modulación. A continuación, se describe el modelo teórico que relaciona las señales de salida con los valores de los coeficientes de reflexión de las cargas. El modelo planteado es una extensión del modelo de seis puertos mostrado en (2.42).

\subsubsection{Modelo Teórico}

Para obtener la relación entre las salidas $b_{2}$ y $b_{3}$ y la entrada $a_{1}$ del modulador se utilizan los parámetros $S$ de la estructura de siete puertos, los cuales se obtienen a partir de los parámetros del acoplador híbrido en cuadratura y el divisor Wilkinson (2.9) utilizando diagrama de grafos como se presenta en el Capítulo 2. Considerando la numeración de los 


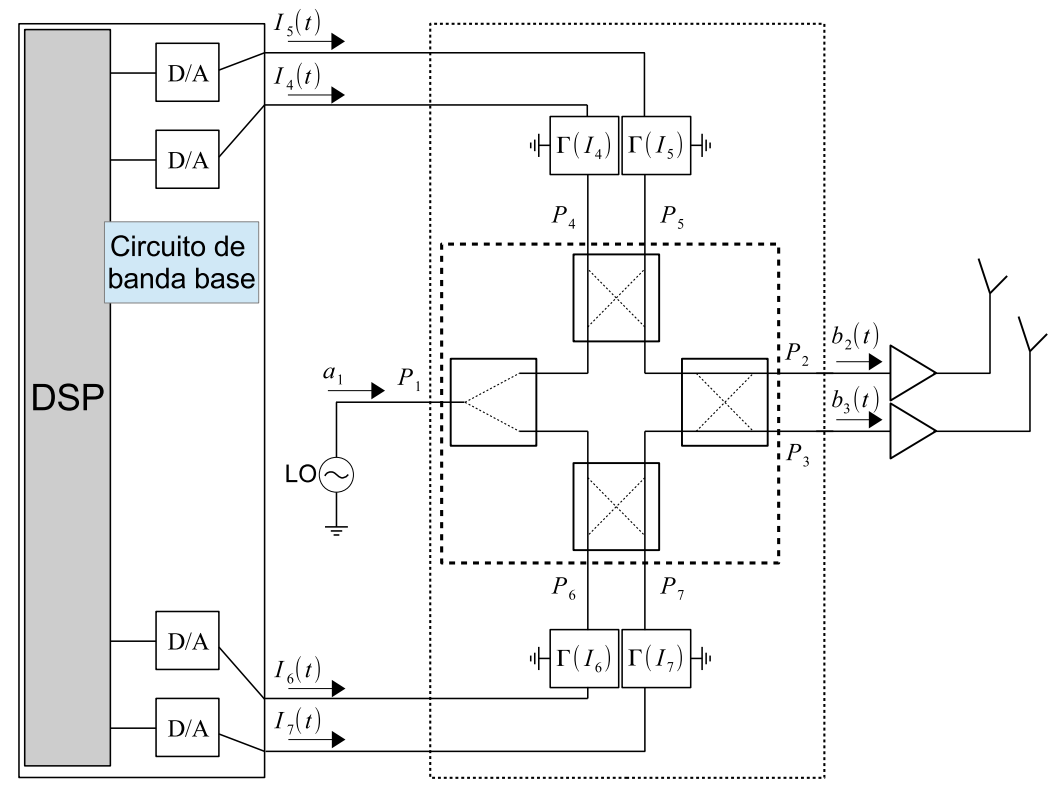

Figura 3.1: Modulador basado en una red de siete puertos.

puertos en la Figura 3.1 la matriz resulta

$$
S=\frac{1}{2}\left[\begin{array}{ccccccc}
0 & 0 & 0 & -1 & j & -1 & j \\
0 & 0 & 0 & j & -1 & 1 & j \\
0 & 0 & 0 & 1 & j & j & -1 \\
-1 & j & 1 & 0 & 0 & 0 & 0 \\
j & -1 & j & 0 & 0 & 0 & 0 \\
-1 & 1 & j & 0 & 0 & 0 & 0 \\
j & j & -1 & 0 & 0 & 0 & 0
\end{array}\right]
$$

La mayoría de los elementos en (3.1) coinciden con los de (2.10), a excepción de los parámetros relacionados con el puerto definido como $P_{2}$. La relación entre las ondas incidentes y reflejadas en los puertos de la red resulta

$$
\left[\begin{array}{c}
b_{1} \\
\vdots \\
b_{7}
\end{array}\right]=\left[\begin{array}{cc}
0^{3 \times 3} & S_{r} \\
S_{r}^{T} & 0^{4 \times 4}
\end{array}\right]\left[\begin{array}{c}
a_{1} \\
\vdots \\
a_{7}
\end{array}\right]
$$

donde los parámetros en rojo en (3.1) definen la siguiente matriz

$$
S_{r}=\frac{1}{2}\left[\begin{array}{cccc}
-1 & j & -1 & j \\
j & -1 & 1 & j \\
1 & j & j & -1
\end{array}\right]
$$

En la posición de las cargas variables, la relación entre la señal incidente y reflejada resulta $a_{i}=\Gamma_{i} b_{i}$ con $i \in\{4,5,6,7\}$, donde $\Gamma_{i}$ es el coeficiente de reflexión debido a la presencia de la impedancia en el puerto $i$. Para los cuatro puertos $P_{4}-P_{7}$ se puede escribir la siguiente relación

$$
\left[\begin{array}{l}
a_{4} \\
a_{5} \\
a_{6} \\
a_{7}
\end{array}\right]=\Gamma_{r}\left[\begin{array}{l}
b_{4} \\
b_{5} \\
b_{6} \\
b_{7}
\end{array}\right]
$$


donde $\Gamma_{r}=\operatorname{diag}\left(\Gamma_{4}, \ldots, \Gamma_{7}\right)$ es una matriz diagonal. Luego, introduciendo (3.4) en (3.2) se obtiene

$$
\left[\begin{array}{l}
b_{1} \\
b_{2} \\
b_{3} \\
b_{4} \\
b_{5} \\
b_{6} \\
b_{7}
\end{array}\right]=\left[\begin{array}{cc}
0 & S_{r} \\
S_{r}^{T} & 0
\end{array}\right]\left[\begin{array}{cc}
I_{3} & 0 \\
0 & \Gamma_{r}
\end{array}\right]\left[\begin{array}{l}
a_{1} \\
a_{2} \\
a_{3} \\
b_{4} \\
b_{5} \\
b_{6} \\
b_{7}
\end{array}\right]
$$

donde $I_{3}$ es una matriz identidad de dimensión $3 \times 3$. La expresión anterior se puede separar en dos partes

$$
\begin{gathered}
{\left[\begin{array}{l}
b_{1} \\
b_{2} \\
b_{3}
\end{array}\right]=S_{r} \Gamma_{r}\left[\begin{array}{l}
b_{4} \\
b_{5} \\
b_{6} \\
b_{7}
\end{array}\right]} \\
{\left[\begin{array}{l}
b_{4} \\
b_{5} \\
b_{6} \\
b_{7}
\end{array}\right]=S_{r}^{T}\left[\begin{array}{l}
a_{1} \\
a_{2} \\
a_{3}
\end{array}\right]}
\end{gathered}
$$

Remplazando el vector $\left[b_{4} \cdots b_{7}\right]^{T}$ de $(3.7)$ en (3.6) se obtiene

$$
\left[\begin{array}{l}
b_{1} \\
b_{2} \\
b_{3}
\end{array}\right]=S_{r} \Gamma_{r} S_{r}^{T}\left[\begin{array}{l}
a_{1} \\
a_{2} \\
a_{3}
\end{array}\right] .
$$

El factor $S_{r} \Gamma_{r} S_{r}^{T}$ representa la matriz de parámetros $\mathrm{S}$ de un dispositivo de tres puertos formado por la red de siete puertos y las cuatro impedancias variables. Utilizando los valores en (3.3) y considerando que los dispositivos conectados en los puertos de salida $P_{2}$ y $P_{3}$ están perfectamente adaptados a la impedancia característica $Z_{0}$ de la red, entonces $a_{2}=a_{3}=0$ y la parte derecha de (3.8) se reduce a

$$
\left[\begin{array}{l}
b_{1} \\
b_{2} \\
b_{3}
\end{array}\right]=\frac{a_{1}}{4}\left[\begin{array}{cccc}
-1 & 1 & -1 & 1 \\
-j & -j & -1 & -1 \\
-1 & -1 & -j & -j
\end{array}\right] \gamma
$$

donde $\gamma$ es un vector columna formado por los elementos diagonales de la matriz $\Gamma_{r}$.

\section{Pérdidas por Retorno}

La primera fila en (3.9) muestra la relación entre la onda incidente y reflejada en el puerto de entrada $P_{1}$, lo cual define las pérdidas por retorno del sistema como

$$
\begin{aligned}
\mathrm{RL} & =20 \log \frac{\left|a_{1}\right|}{\left|b_{1}\right|} \\
& =-20 \log \left\{\frac{1}{4}\left[\begin{array}{cccc}
-1 & 1 & -1 & 1
\end{array}\right] \gamma\right\} \\
& =-20 \log \frac{\left|\Gamma_{4}-\Gamma_{5}+\Gamma_{6}-\Gamma_{7}\right|}{4}
\end{aligned}
$$


donde $0<\left|\Gamma_{4}-\Gamma_{5}+\Gamma_{6}-\Gamma_{7}\right|<4$. Dichas pérdidas pueden ser grandes o pequeñas dependiendo de la relación entre los coeficientes de reflexión. Por ejemplo, si $\mid \Gamma_{4}-\Gamma_{5}+$ $\Gamma_{6}-\Gamma_{7} \mid=4$, las pérdidas son $\mathrm{RL}=0 \mathrm{~dB}$ es decir, toda la potencia de entrada se refleja y retorna al oscilador local. Por el contrario, cuando $\Gamma_{4}-\Gamma_{5}+\Gamma_{6}-\Gamma_{7}=0$, las pérdidas por retorno son $\mathrm{RL} \rightarrow \infty \mathrm{dB}$, lo que indica que la potencia reflejada es nula y por lo tanto el puerto de entrada del modulador está perfectamente adaptado al oscilador local.

\section{Señales de Salida}

Considerando solo las salidas $b_{2}$ y $b_{3}$ en (3.9) se obtiene la siguiente expresión

$$
\left[\begin{array}{l}
b_{2} \\
b_{3}
\end{array}\right]=\frac{a_{1}}{4}\left[\begin{array}{ll}
-j & -1 \\
-1 & -j
\end{array}\right]\left[\begin{array}{l}
\Gamma_{4}+\Gamma_{5} \\
\Gamma_{6}+\Gamma_{7}
\end{array}\right]
$$

donde la matriz que relaciona las salidas con los coeficientes de reflexión es no-singular y por lo tanto se puede obtener el problema inverso

$$
\left[\begin{array}{c}
\Gamma_{4}+\Gamma_{5} \\
\Gamma_{6}+\Gamma_{7}
\end{array}\right]=2\left[\begin{array}{cc}
j & -1 \\
-1 & j
\end{array}\right]\left[\begin{array}{c}
\frac{b_{2}}{a_{1}} \\
\frac{b_{3}}{a_{1}}
\end{array}\right]
$$

La ecuación (3.12) presenta los valores de los coeficientes de reflexión de las cargas variables en función de las salidas $\left[b_{2} / a_{1}, b_{3} / a_{1}\right]^{T}$ deseadas. Debido a la restricción de magnitud de los coeficientes de reflexión $0 \leq\left|\Gamma_{i}\right|<1$ se establece una restricción en la amplitud máxima de las salidas, lo cual afecta a la eficiencia de conversión del modulador.

\section{Eficiencia de Conversión}

La eficiencia de conversión del modulador propuesto se define como la relación entre la potencia de la señal/es de salida $\left(P_{s}\right)$ y la potencia de la señal de OL en el puerto de entrada $\left(P_{O L}\right)$

$$
\eta=\frac{P_{s}}{P_{O L}}=\frac{\left|b_{2}\right|^{2}+\left|b_{3}\right|^{2}}{\left|a_{1}\right|^{2}} .
$$

Calculando $\left|b_{2}\right|^{2}+\left|b_{3}\right|^{2}$ a partir de (3.11), la eficiencia se puede escribir en términos de los coeficientes de reflexión en los puertos $P_{4}-P_{7}$ como

$$
\eta=\frac{1}{8}\left(\left|\Gamma_{4}+\Gamma_{5}\right|^{2}+\left|\Gamma_{6}+\Gamma_{7}\right|^{2}\right) .
$$

Dependiendo de la relación entre los coeficientes de reflexión dentro del módulo, el valor de la eficiencia puede variar entre $0 \%$ y $100 \%$. Por ejemplo, solo cuando se cumple la relación $\Gamma_{4}=\Gamma_{5}$ y $\Gamma_{6}=\Gamma_{7}$ y el valor de los coeficientes de reflexión varía alrededor del circulo unitario $(|\Gamma|=1)$, entonces la eficiencia resulta $\eta=100 \%$. En estas condiciones también se cumple que RL $\rightarrow \infty$ en (3.10).

A modo de comparación se presenta la eficiencia del modulador de seis puertos, que siguiendo la definición (3.13) resulta

$$
\eta=\frac{P_{s}}{P_{O L}}=\frac{\left|b_{2}\right|^{2}}{\left|a_{1}\right|^{2}} .
$$


A partir de (2.43) se obtiene la eficiencia en función de los coeficientes de reflexión

$$
\eta=\frac{1}{16}\left(\left|\Gamma_{Q}\right|^{2}+\left|\Gamma_{I}\right|^{2}+2 \Im\left\{\left(\Gamma_{Q}\right)\left(\Gamma_{I}\right)^{*}\right\}\right)
$$

donde la función $\Im\{x\}$ calcula la parte imaginaria de $x, \Gamma_{I}=\Gamma_{3}+\Gamma_{4}$ y $\Gamma_{Q}=\Gamma_{5}+\Gamma_{6}$ (estos coeficientes siguen la numeración de puertos planteada para el modulador de seis puertos). Notar que, la eficiencia del modulador de seis puertos depende del factor $\Im\left\{\left(\Gamma_{Q}\right)\left(\Gamma_{I}\right)^{*}\right\}$ cuyo valor máximo ocurre cuando $\Gamma_{I}$ y $\Gamma_{Q}$ están en cuadratura. Por el contrario, al utilizar la configuración típica de los moduladores de seis puertos $\Im\left\{\left(\Gamma_{Q}\right)\left(\Gamma_{I}\right)^{*}\right\}=0$ debido a que $\Gamma_{I}$ y $\Gamma_{Q}$ están en fase y contrafase. Por este último, a nuestro entendimiento, todos los moduladores de seis puertos presentan una eficiencia inferior a $50 \%$. Por ejemplo, en [34] los resultados de eficiencia teórica son $\eta=38 \%$ (ver (42) en [34]), y en [33] la eficiencia es cercana a $50 \%$ debido a que las cargas son llaves cuyo coeficiente de reflexión varía de $1 \mathrm{a}-1$.

\subsubsection{Ejemplos de Aplicación}

En esta sección se formulan las salida del modulador de siete puertos para los ejemplos de diversidad polarimétrica y espacial desarrollados al final del Capítulo 2.

\section{Arreglo de Antenas}

Para este ejemplo, las salidas $b_{2}$ y $b_{3}$ del modulador de siete puertos se conectan a dos antenas separadas por una distancia $d=\lambda / 2$. Como consecuencia, es posible controlar el haz de transmisión del arreglo estableciendo una diferencia de fase $\Delta \phi$ entre las señales de las antenas. Para transmitir una señal en la dirección $\theta$ se configuran las señales de la siguiente manera

$$
\left[\begin{array}{l}
b_{2} \\
b_{3}
\end{array}\right]=\frac{a_{1}}{\sqrt{2}} C\left[\begin{array}{c}
1 \\
e^{j \pi \sin \theta}
\end{array}\right]
$$

donde $a_{1}$ es la amplitud compleja de la señal portadora y $C$ es la envolvente compleja de la señal transmitida y se define como

$$
C=\sqrt{\frac{\left|b_{2}\right|^{2}+\left|b_{3}\right|^{2}}{\left|a_{1}\right|^{2}}} e^{j\left(\angle b_{2}-\angle a_{1}\right)} .
$$

Remplazando (3.17) en (3.12) se obtiene

$$
\left[\begin{array}{c}
\Gamma_{4}+\Gamma_{5} \\
\Gamma_{6}+\Gamma_{7}
\end{array}\right]=\sqrt{2} C\left[\begin{array}{cc}
j & -1 \\
-1 & j
\end{array}\right]\left[\begin{array}{c}
1 \\
e^{j \pi \sin \theta}
\end{array}\right] .
$$

Notar que, la envolvente $C$ es común a ambos factores $\Gamma_{4}+\Gamma_{5}$ y $\Gamma_{6}+\Gamma_{7}$ y no influye en la relación entre ellos, que resulta

$$
\frac{\Gamma_{4}+\Gamma_{5}}{\Gamma_{6}+\Gamma_{7}}=\frac{j-e^{j \pi \sin \theta}}{-1+j e^{j \pi \sin \theta}}
$$

A partir de (3.19) se obtiene el valor de la eficiencia (3.14) en función del ángulo $\theta$ como sigue

$$
\eta=\frac{\rho^{2} / 4}{1+\sin |\pi \sin \theta|} \quad-30^{\circ} \leq \theta \leq 30^{\circ} .
$$


donde $\rho$ es el valor máximo entre los dos factores $\left|\Gamma_{4}+\Gamma_{5}\right|$ o $\left|\Gamma_{6}+\Gamma_{7}\right|$. La expresión (3.21) se demuestra en el Apéndice A. La Figura 3.2 presenta los valores de la eficiencia en función del ángulo de propagación.

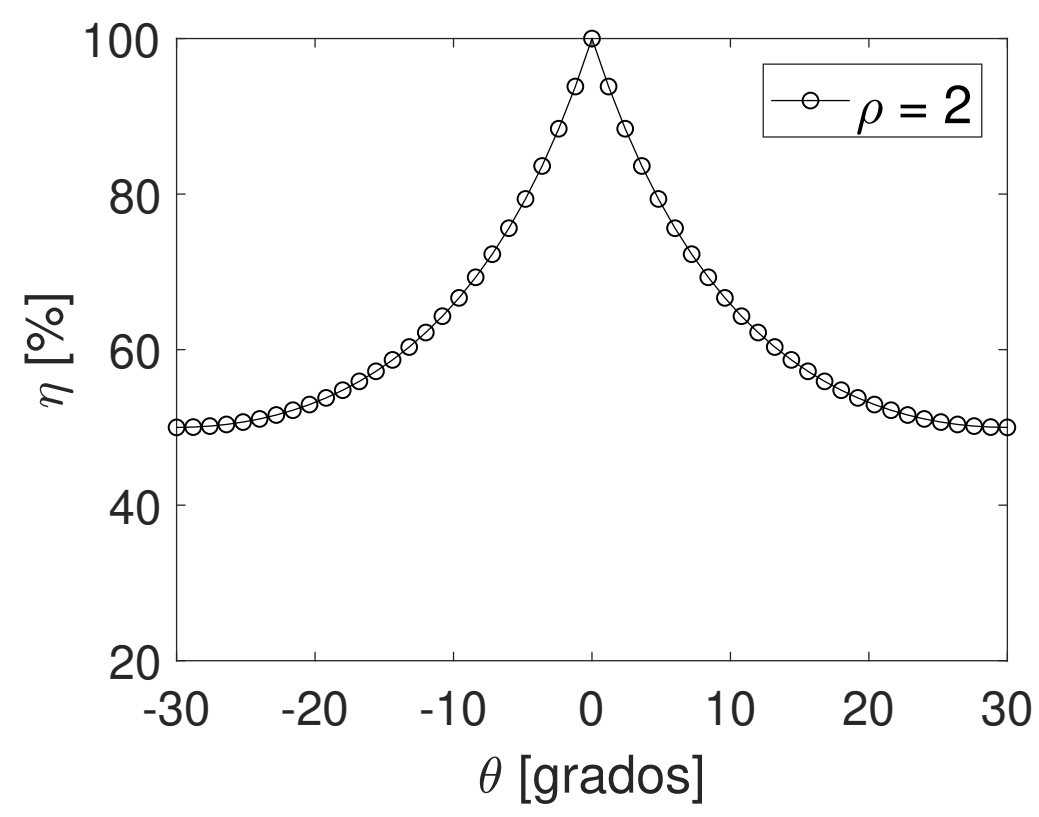

Figura 3.2: Eficiencia teórica en función del ángulo $\theta$.

\section{Antena de Doble Polarización}

Las salidas $b_{2}$ y $b_{3}$ del modulador de siete puertos se conectan a una antena de dos puertos capaz de irradiar dos señales con distinta polarización: una lineal vertical $b_{V}$ y la otra horizontal $b_{H}$. Considerando que el puerto $P_{2}$ se conecta al canal horizontal y el puerto $P_{3}$ se conecta al canal vertical, entonces a partir de (2.45) la señal transmitida por la antena resulta

$$
b=\left[\begin{array}{l}
b_{2} \\
b_{3}
\end{array}\right]=a_{1} C\left[\begin{array}{cc}
\cos \alpha & -\sin \alpha \\
\sin \alpha & \cos \alpha
\end{array}\right]\left[\begin{array}{c}
\cos \beta \\
j \sin \beta
\end{array}\right]
$$

donde $C$ se define como (3.18). Dividiendo la expresión anterior por $a_{1}$ y remplazando el vector $\left[\frac{b_{2}}{a_{1}}, \frac{b_{3}}{a_{1}}\right]^{T}$ en (3.12) se obtiene la expresión que relaciona los valores de las cargas variables con la envolvente compleja y los ángulos de polarización de la señal transmitida

$$
\left[\begin{array}{c}
\Gamma_{4}+\Gamma_{5} \\
\Gamma_{6}+\Gamma_{7}
\end{array}\right]=2 C\left[\begin{array}{cc}
j & -1 \\
-1 & j
\end{array}\right]\left[\begin{array}{cc}
\cos \alpha & -\sin \alpha \\
\sin \alpha & \cos \alpha
\end{array}\right]\left[\begin{array}{c}
\cos \beta \\
j \sin \beta
\end{array}\right] .
$$

Desarrollando el producto entre matrices y vectores en la expresión anterior se obtiene

$$
\left[\begin{array}{c}
\Gamma_{4}+\Gamma_{5} \\
\Gamma_{6}+\Gamma_{7}
\end{array}\right]=2 C\left[\begin{array}{c}
(\cos \beta-\sin \beta) e^{j(\alpha+\pi / 2)} \\
(\cos \beta+\sin \beta) e^{-j(\alpha-\pi)}
\end{array}\right]
$$

Calculando el cociente entre $\Gamma_{4}+\Gamma_{5}$ y $\Gamma_{6}+\Gamma_{7}$ en (3.24) se elimina la dependencia con $C$ y se definen las siguiente ecuaciones

$$
\alpha=\frac{\pi}{4}+\frac{1}{2}\left(\angle\left(\Gamma_{4}+\Gamma_{5}\right)-\angle\left(\Gamma_{6}+\Gamma_{7}\right)\right)
$$


y

$$
\begin{aligned}
\frac{\left|\Gamma_{4}+\Gamma_{5}\right|}{\left|\Gamma_{6}+\Gamma_{7}\right|} & =\frac{1-\tan \beta}{1+\tan \beta} \\
\tan \beta & =\frac{\left|\Gamma_{6}+\Gamma_{7}\right|-\left|\Gamma_{4}+\Gamma_{5}\right|}{\left|\Gamma_{6}+\Gamma_{7}\right|+\left|\Gamma_{4}+\Gamma_{5}\right|}
\end{aligned}
$$

Por lo tanto, un determinado estado de polarización definido por $\alpha$ y $\beta$ se configura seleccionando adecuadamente la relación de magnitudes y la diferencia de fase entre $\Gamma_{4}+\Gamma_{5}$ y $\Gamma_{6}+\Gamma_{7}$. Asimismo, el parámetro $C$ que define la modulación de la señal transmitida, se puede obtener a partir de la magnitud y fase en común entre los factores $\Gamma_{4}+\Gamma_{5} \mathrm{y}$ $\Gamma_{6}+\Gamma_{7}$ que no influye en el estado de polarización.

Para este ejemplo, la eficiencia (3.14) la cual se demuestra en el Apéndice B, resulta

$$
\eta=\frac{\rho^{2} / 4}{1+2 \cos \beta \sin |\beta|} \quad-45^{\circ} \leq \beta \leq 45^{\circ} .
$$

Esta expresión demuestra que la eficiencia depende únicamente de $\beta$ y $\rho$, donde $\rho \in(0,2)$. En la Figura 3.3 se presenta la eficiencia en función del ángulo de elipticidad $\beta$. Notar que

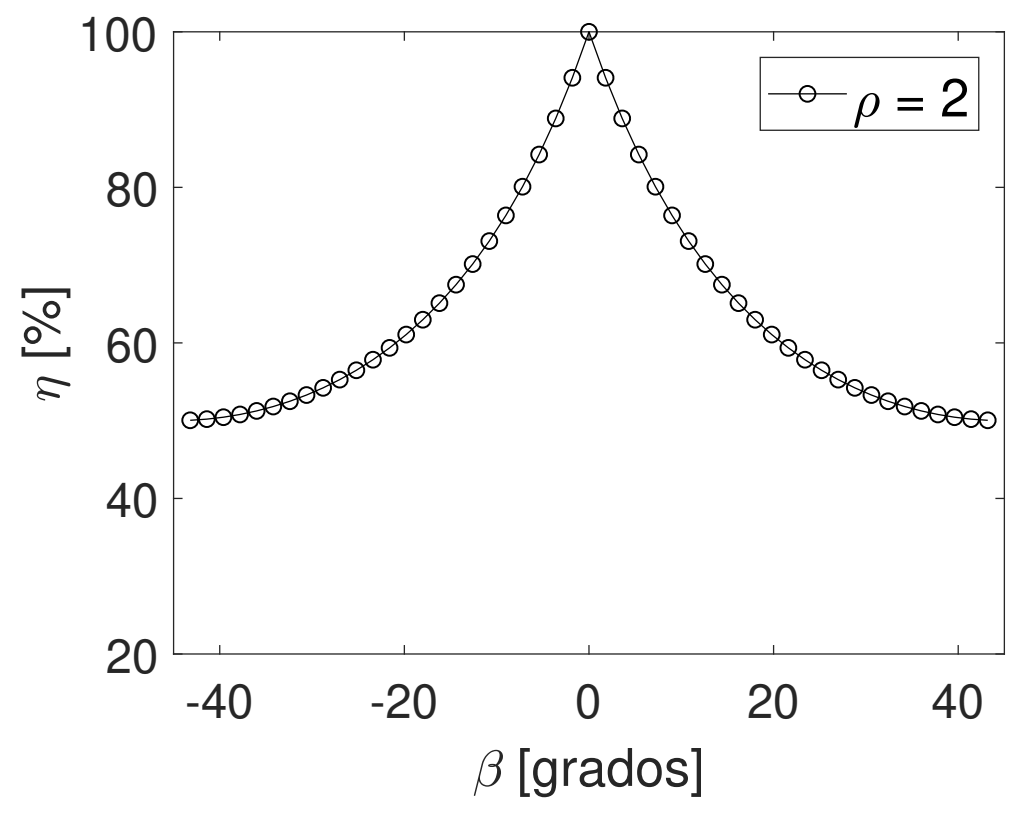

Figura 3.3: Eficiencia teórica en función del ángulo $\beta$.

el valor máximo de la eficiencia se alcanza cuando la polarización es lineal $\left(\beta=0^{\circ}\right)$. Esto ocurre como consecuencia de utilizar una antena de polarización lineal. En un ejemplo diferente, en el que la antena utilizada transmite polarización circular a izquierda y a derecha, la condición de máxima eficiencia corresponderá al caso circular $\left(\beta= \pm 45^{\circ}\right)$. A continuación se presenta un prototipo del modulador de siete puertos considerando este ejemplo de diversidad polarimétrica. 


\subsection{Descripción del Prototipo}

Para validar la teoría desarrollada en la secciones anteriores, se diseñó e implementó un prototipo modular incluyendo la red de siete puertos, las cargas variables, y la antena de dos puertos. Todas las partes se diseñaron e implementaron en microtira, a una frecuencia de operación de 1575,42 MHz, en placas con sustrato de FR-4 de espesor 1,542 mm, e impedancia característica $Z_{0}=50 \Omega$. La Figura 3.4 muestra los dispositivos implementados.
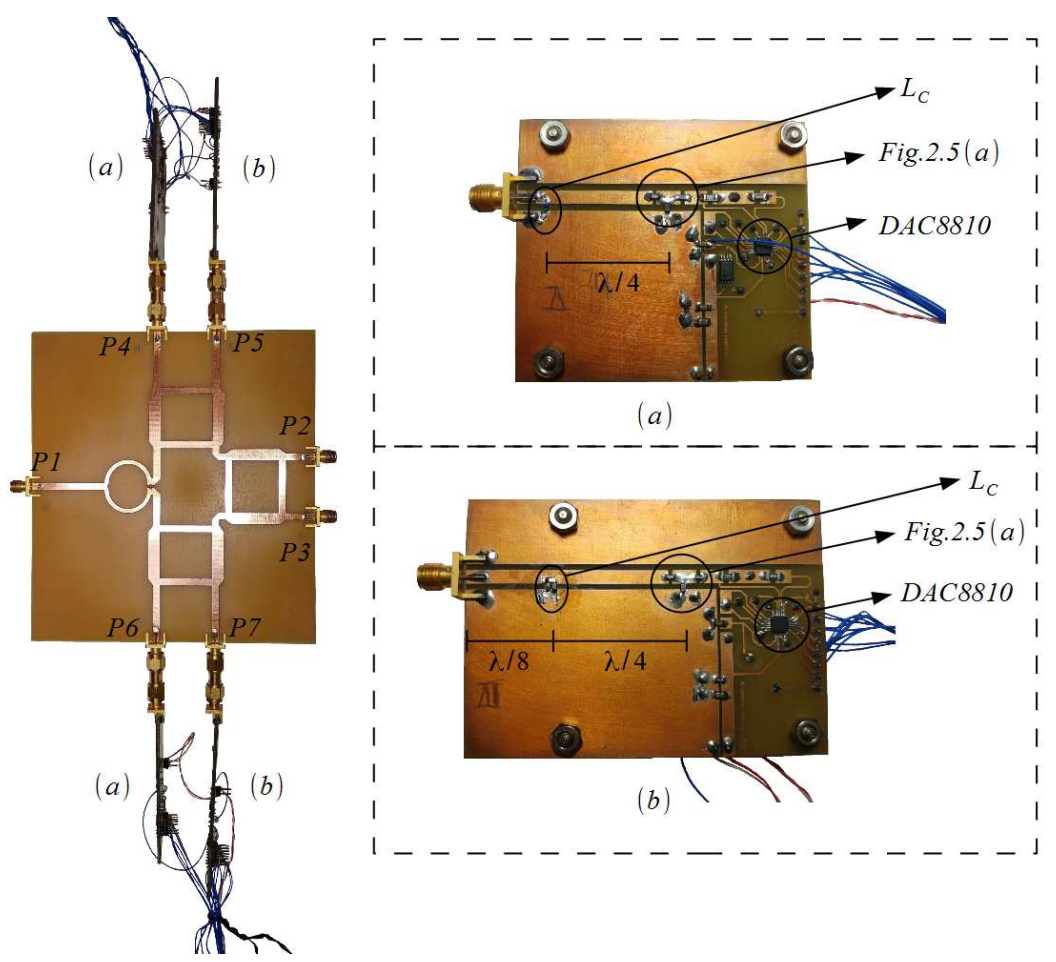

Figura 3.4: Prototipo implementado para operar a la frecuencia 1575,42 MHz. Modulador completo (izquierda), cargas variables (derecha).

\subsection{1. $\quad$ Red de Siete Puertos}

Para determinar las dimensiones de la estructura de siete puertos se simuló el circuito en el software CST microwave studio. Los parámetros S resultantes de la simulación se muestran en la Figura 3.5. Luego, se realizó la implementación de la red en una placa de $15 \times 13 \mathrm{~cm}$ y terminando todos los puertos con conectores SMA hembra. Las mediciones de los parámetros S se realizaron utilizando un analizador MS46122B de Anritsu. En la Figura 3.5 se muestran los parámetros medidos y simulados en función de la frecuencia. Solo se presentan unos pocos parámetros, los cuales muestran las carcterísticas de aislación $s_{45}$, de adaptación $s_{11}$, y de transferencia $s_{41}$ y $s_{42}$ de la red. Se observa en la Figura una buena correspondencia entre los valores medidos y los valores obtenidos por simulación. En la Figura 3.4 se muestra la red de siete puertos implementada junto las cargas variables las cuales se describen en la siguiente sección. 


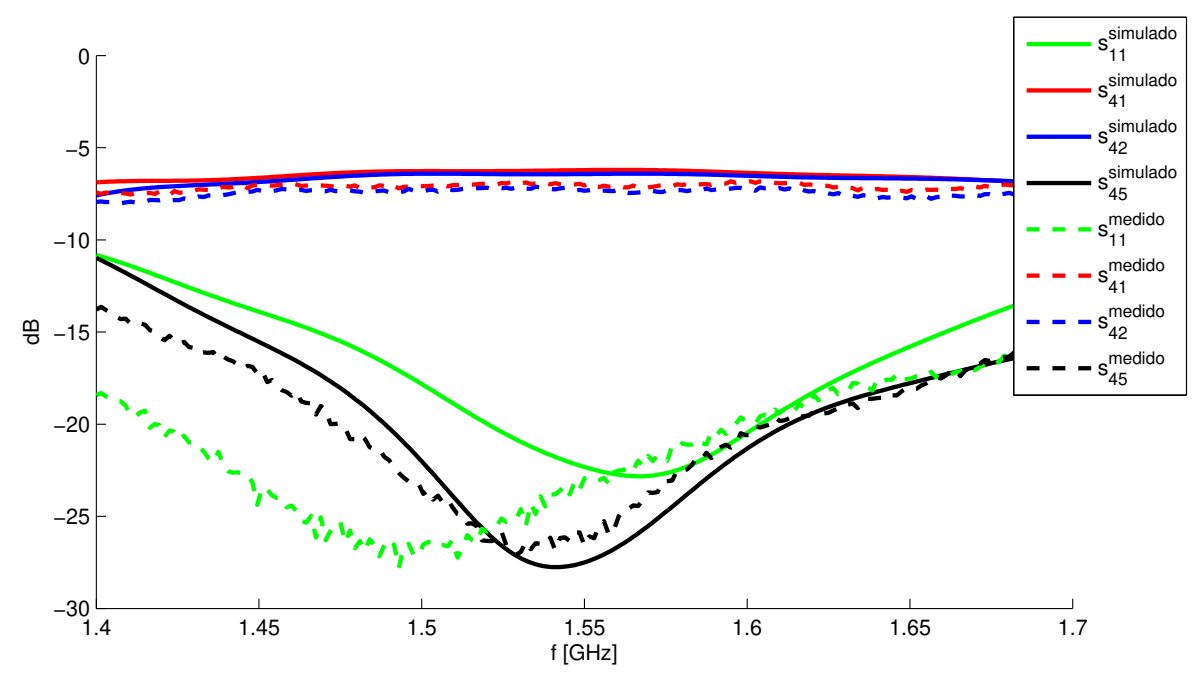

Figura 3.5: Magnitud de los parámetros S de la red de siete puertos.

\subsubsection{Impedancias Variables}

Entre las diferentes alternativas para el diseño de las cargas, se eligió un enfoque basado en diodos PIN debido a su comportamiento en frecuencias de microondas. Cuando el diodo se encuentra polarizado con una corriente en directa este se comporta como una resistencia variable con un amplio rango de valores, típicamente entre 1 y $10000 \Omega$ cuando la corriente varía entre el valor máximo y cero. Por consiguiente, cuando el diodo está conectado a un puerto cuya impedancia característica es $Z_{0}=50 \Omega$, el coeficiente de reflexión definido por la expresión $(2.36)$ toma valores reales que varían entre $(-1,1)$. Por otro lado, cuando se conecta una línea de transmisión de $\lambda / 8$ entre el diodo y el puerto, el coeficiente de reflexión en el puerto de la red varia entre $(-j, j)$ debido al desplazamiento de fase de $\pi / 2$ introducido por la línea. En la Figura 3.6(a) y (b) se presentan los circuitos esquemáticos de las cargas. Si se conectan las impedancias de valor real a los puertos $P_{4}$ y $P_{6}$ y las de valor imaginario en los puertos $P_{5}$ y $P_{7}$, se puede controlar la parte real e imaginaria de las cantidades $\Gamma_{4}+\Gamma_{5}$ y $\Gamma_{6}+\Gamma_{7}$. La corriente de polarización $I_{i}$ $(i \in\{4,5,6,7\})$ que varía la impedancia de entrada del diodo se aplica sobre el ánodo, a través del choque $L_{C H}$, mientras el cátodo se conecta a tierra. El capacitor $C_{D C}$ aparece como un circuito abierto para la corriente de polarización, pero como un cortocircuito para la señal portadora (en frecuencias de microondas).

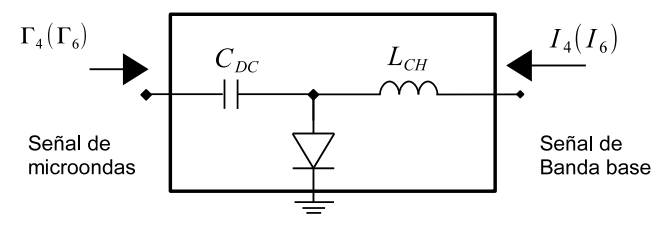

(a)

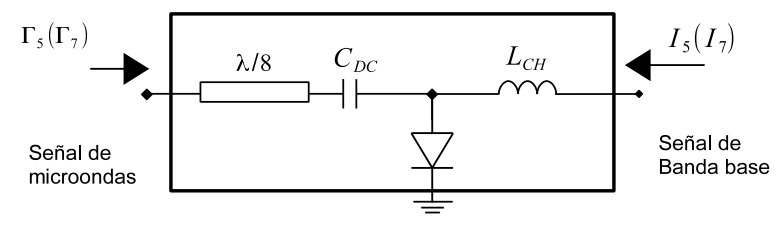

(b)

Figura 3.6: Circuito de las cargas variables

Un modelo simplificado del diodo PIN polarizado en directa consiste en la conexión serie 
entre la resistencia controlada y una inductancia parásita.

$$
Z=R(I)+j \omega L
$$

El valor de la resistencia depende de la corriente de polarización $I$ con el comportamiento $R(I)=K / I^{x}$. Las constantes $K$ y $x$ son parámetros de ajuste. En general, la influencia de la capacidad parásita es despreciable cuando el diodo está polarizado en directa. Sin embargo, en frecuencias muy altas (superiores a $5 \mathrm{GHz}$ ) su influencia puede ser relevante, limitando el rango de $R$. Finalmente, el coeficiente de reflexión resulta

$$
\Gamma_{d}(I)=\frac{R(I)+j \omega L-Z_{0}}{R(I)+j \omega L+Z_{0}} .
$$

En la Figura 3.7 muestra un diagrama de Smith con los valores del coeficiente de reflexión para 3 valores de inductancia parásita. Por cada valor de $L$, se varía la resistencia entre 1 y $10000 \Omega$. Notar que a medida que aumenta la inductancia parásita, se desvían los valores del coeficiente de reflexión respecto al eje real. Esto reduce el área de cobertura formada por la suma de los coeficientes $\Gamma_{4}+\Gamma_{5}$, lo cual impacta en el rango de valores de salida del modulador. Por este motivo resulta importante compensar el efecto de la inductancia parásita.

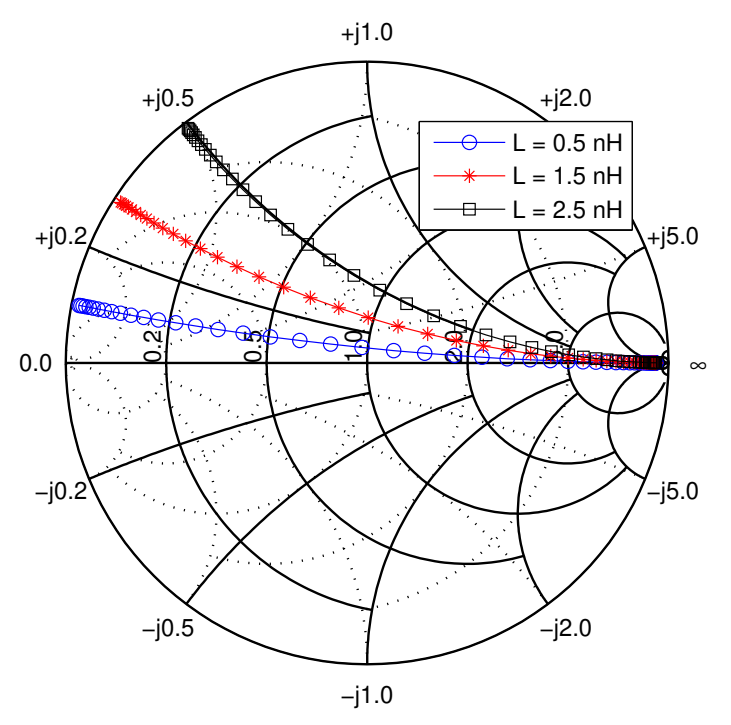

Figura 3.7: Simulación de $\Gamma_{d}(I)$ para diferentes inductancias parásitas

\section{Método de Compensación}

Dicho método consiste en el agregado de un elemento reactivo $L_{c}$ conectado a una distancia de $\lambda / 4$ del diodo. Este componente puede ser un elemento discreto o una línea de transmisión en cortocircuito o circuito abierto dependiendo de la frecuencia de operación. También se puede compensar la inductancia parásita agregando un capacitor en serie. Sin embargo, se elige la versión paralelo por que es más simple de construir.

Para calcular la impedancia a una distancia $l$ de la carga (diodo) en una linea de transmisión se utiliza la siguiente expresión [39]

$$
Z(l)=Z_{0} \frac{Z+Z_{0} \tan \beta l}{Z_{0}+Z \tan \beta l}
$$


donde $Z$ es la impedancia de entrada del diodo dada por (3.28). Si $l=\lambda / 4$ la línea de de transmisión opera como un inversor de impedancia, es decir que (3.30) se reduce a

$$
Z(\lambda / 4)=\frac{Z_{0}^{2}}{Z}
$$

y la admitancia en el punto de compensación resulta

$$
Y=\frac{Z}{Z_{0}^{2}}
$$

Para cancelar la parte imaginaria de (3.32), el elemento en paralelo debe ser un inductor de valor

$$
L_{c}=\frac{Z_{0}^{2}}{\omega^{2} L}
$$

Para obtener un valor de $L_{c}$ se realiza una simulación de la admitancia (3.32) utilizando los parámetros $\mathrm{S}$ que brinda el fabricante del diodo. El esquemático completo de las cargas variables se muestra en la Figura 3.8.

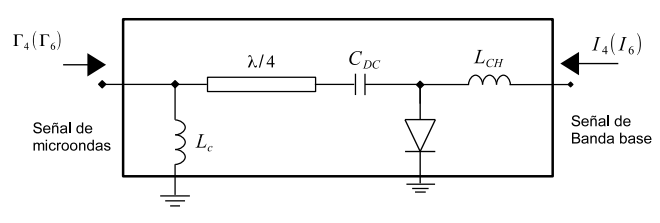

(a)

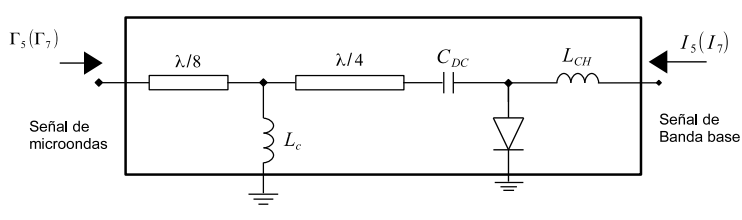

(b)

Figura 3.8: Circuito de las cargas variables compensadas

\section{Implementación}

Los circuitos mostrados en la Figura 3.8 se desarrollan junto con las correspondientes fuentes de corriente en una misma placa como se muestra en la parte derecha de la Figura 3.4. Para la implementación, los diodos PIN son BAP70-03 de NXP Semiconductors y las fuentes de corriente son conversores digitales analógicos (DACs) ADN8810 de Analog Devices. Una computadora controla los DACs transmitiendo palabras de 12 bits a través de una interfaz serie (SPI). Las corrientes pueden variar entre 0 - $40 \mathrm{~mA}$ con una resolución de $\Delta I=9,76 \mu \mathrm{A}$ (12-bits). El tiempo de establecimiento de la corriente es de $3 \mu \mathrm{s}$, lo que limita la tasa de cambio de los valores del coeficiente de reflexión. Otros componentes son $L_{C H}=33 \mathrm{nH}, C_{D C}=180 \mathrm{pF}$ y $L_{c}=11 n \mathrm{H}$.

En la Figura 3.4 se muestran dos de las cuatro impedancias implementadas, dado que las otras dos son iguales. Tenga en cuenta que uno de los circuitos es mas grande que el otro debido a que incluye la línea de transmisión de $\lambda / 8(l=11,9 \mathrm{~mm})$. El diseño de las cargas se validó midiendo el coeficiente de reflexión para distintos valores de corriente utilizando un analizador MS46122B de Anritsu. Las corrientes varían entre [0 - 40] mA, tomando 100 valores espaciados logarítmicamente. Los resultados a la frecuencia 1575,42 MHz son mapeados en el diagrama de Smith como se muestra en la Figura 3.9. 


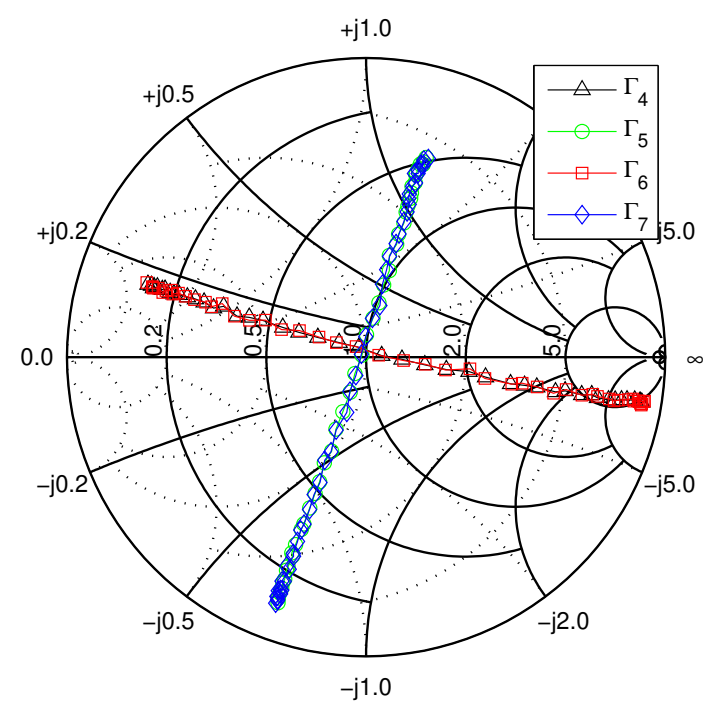

Figura 3.9: Coeficientes de reflexión medidos a 1575,42 MHz.

\subsubsection{Antena}

Finalmente, se desarrollan antenas para realizar una validación práctica del modulador. Se utilizan antenas de microtira que consisten de un parche circular (antenna patch) conectado a dos líneas de transmisión. Los puertos de alimentación de la antena se conectan en cuadratura para excitar un campo electromagnético de polarización líneal con orientaciones ortogonales. Debido a la desadaptación que se produce en los puertos de la antena por la conexión directa, se diseña un transformador de impedancia de $\lambda / 4$ para adaptar los puertos a una frecuencia de operación de 1575,42 MHz [39].

Se realizó el diseño de la antena por simulación en el software CST microwave studio para determinar las dimensiones óptimas del parche circular y del transformador de $\lambda / 4$. En la parte derecha de la Figura 3.10 se muestra el patrón de radiación de la antena simulada aplicando señal en el puerto $P_{V}$. Dicho patrón de radiación se compone principalmente por ondas de polarización lineal vertical (o $45^{\circ}$ dependiendo del sistema de referencia) como se muestra en la parte inferior de la misma Figura. La implementación de la antena se realizó utilizando las dimensiones establecidas por la simulación. El dispositivo se muestra a la izquierda en la Figura 3.10. Esta antena es capaz de generar dos señales con polarización lineal ortogonal con orientación vertical (o $45^{\circ}$ ) y horizontal (o $\left.-45^{\circ}\right)$. En la Figura 3.11 se muestra la magnitud de los parámetros $\mathrm{S}$ simulados y medidos utilizando el analizador MS46122B.

\subsection{Resultados}

La caracterización del modulador de siete puertos se realiza midiendo las señales en los puertos de salida $P_{2}$ y $P_{3}$ para diferentes combinaciones de los coeficientes de reflexión en los puertos $P_{4}$ a $P_{7}$. Para ello, se arma el sistema de medición como muestra la Figura 3.12. La señal portadora de $1575,42 \mathrm{MHz}$ se genera y se inyecta en el puerto de entrada $P_{1}$ utilizando un generador de señal $8648 \mathrm{C}$ de Keysight Technologies. Las señales 

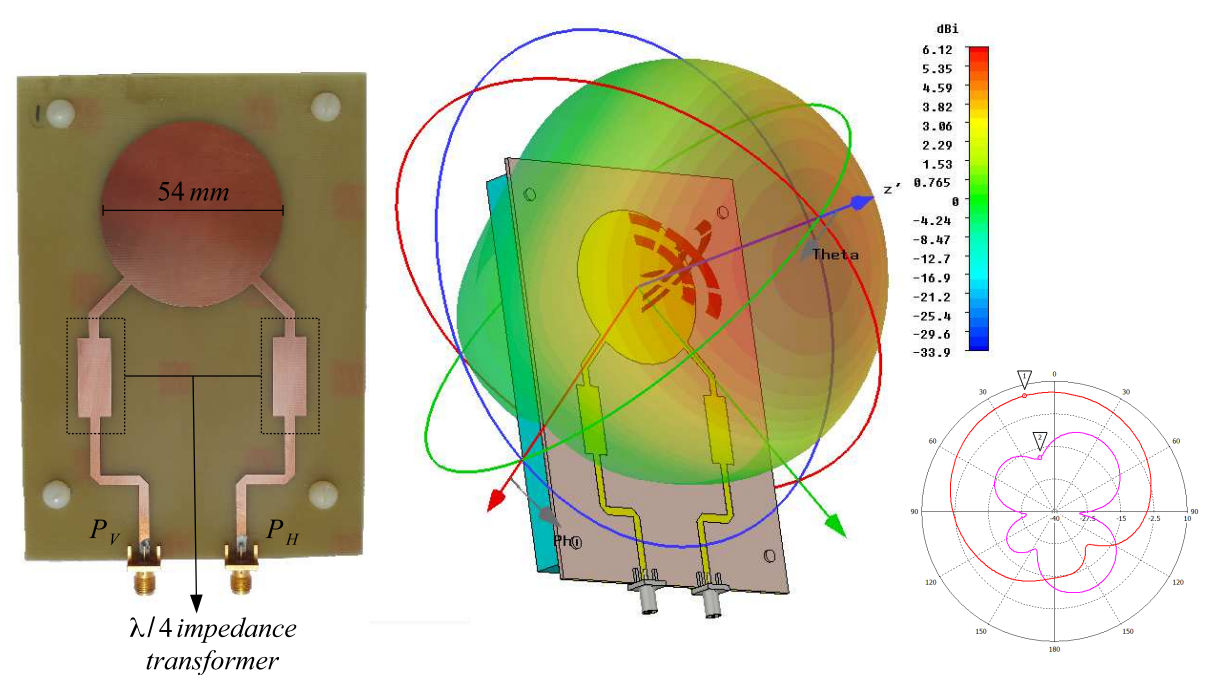

Figura 3.10: Antena implementada y simulada

de salida $b_{2}$ y $b_{3}$ son demoduladas con un transceptor USRP-X300 de Ettus Research que consiste en un dispositivo de radio definida por software de alto rendimiento con dos receptores de cuadratura. Una computadora controla el USRP-X300 y el microcontrolador que configura las fuentes de corriente de las cargas. Los datos se guardan y se procesan en MATLAB. Antes de iniciar la medición, se utilizó el analizador de redes MS42122B para medir las pérdidas de inserción y la fase introducida por las conexiones coaxiales entre los instrumentos y la red de siete puertos, para eliminar su influencia en los resultados.

\subsubsection{Validación del Modelo}

La computadora configura las fuentes de corriente para obtener 100 valores logarítmicamente espaciados entre 0 y $40 \mathrm{~mA}$. Con la intensión de obtener una gran variedad de valores de $b_{2}$ y $b_{3}$ las corrientes se configuran siguiendo el Cuadro 3.1. Los valores $i=0$

Cuadro 3.1:

\begin{tabular}{ccccc} 
estados & $i_{4}[\mathrm{~mA}]$ & $i_{5}[\mathrm{~mA}]$ & $i_{6}[\mathrm{~mA}]$ & $i_{7}[\mathrm{~mA}]$ \\
\hline \hline $1-100$ & $0-40$ & $0-40$ & 0 & 0 \\
$101-200$ & $0-40$ & $0-40$ & 1 & 1 \\
$201-300$ & $0-40$ & $0-40$ & 40 & 40 \\
$301-400$ & 0 & 0 & $0-40$ & $0-40$ \\
$401-500$ & 1 & 1 & $0-40$ & $0-40$ \\
$501-600$ & 40 & 40 & $0-40$ & $0-40$
\end{tabular}

y $i=40 \mathrm{~mA}$ establecen los coeficientes de reflexión de mayor magnitud como muestra la Figura 3.9, mientras que $i=1 \mathrm{~mA}$ corresponde $\Gamma \approx 0$. Por otro lado, para obtener los resultados teóricos se utilizan los valores de los coeficientes de reflexión medidos anteriormente (Figura 3.9) y a partir de ellos se obtiene la respuesta teórica del modulador dada 


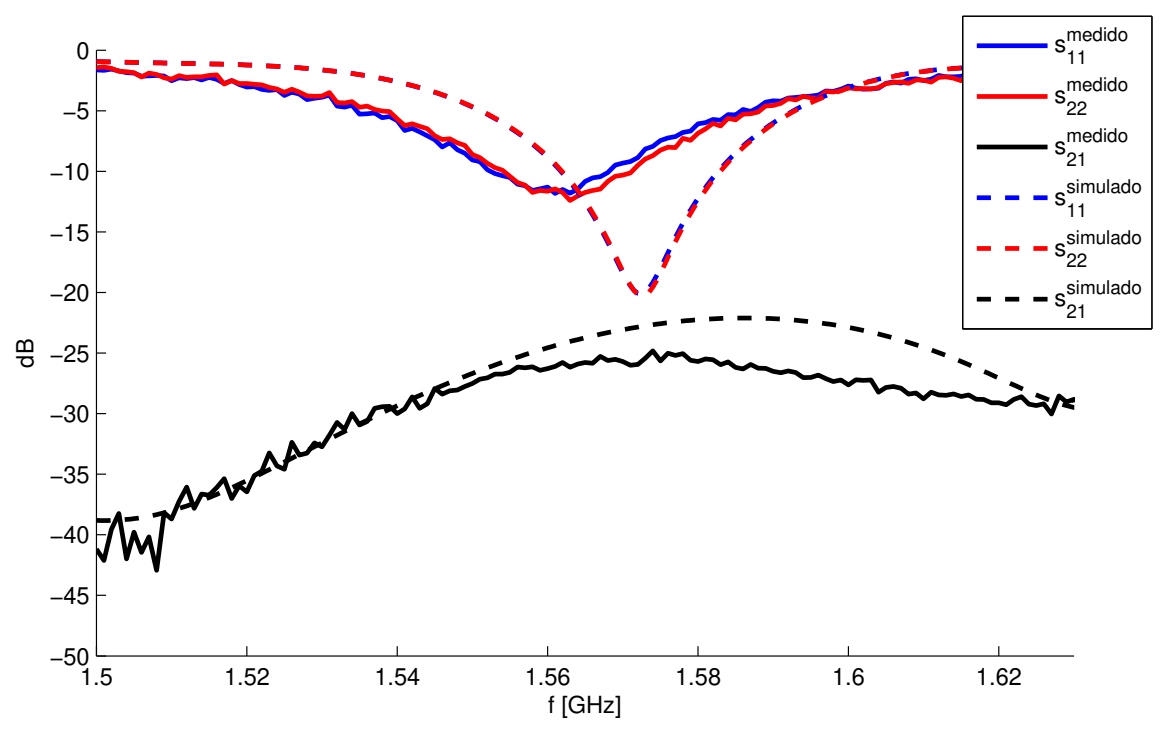

Figura 3.11: Parámetros $\mathrm{S}$ medidos y simulados de la antena de dos puertos, $P_{V}=P_{1}$ y $P_{H}=P_{2}$.

por el modelo (3.11). Los resultados medidos y los resultados teóricos se utilizan para obtener los ángulos de polarización a partir de (2.46) y (2.47). Los valores se presentan en la Figura 3.13(a) y (b). Se observa una buena correspondencia entre los resultados teóricos y medidos del ángulo $\beta$. Sin embargo, la orientación $\alpha$ está sesgada en varios estados, tales como 50, 150, 250, 250, 350, 400-500. En estos casos particulares, se observa que la elipticidad es cercana a $\pm 45^{\circ}$, en cuyo caso la elipse de polarización se convierte en un círculo y el ángulo de orientación es indeterminado, como resulta de calcular $\alpha$ en (2.46). Por otro lado, la envolvente compleja se calcula por (3.18) y se presenta en la Figura $3.13(\mathrm{c})$. La pequeña rotación de los valores medidos respecto al modelo teórico se debe a la longitud eléctrica de los conectores SMA, así como también, de la longitud de los puertos de entrada y salida dentro de la red de siete puertos. Finalmente, la eficiencia se obtiene a partir de los valores medidos calculando la magnitud cuadrada de la envolvente compleja, y ordenando sus valores en función de $\beta$ (de $-45^{\circ}$ a $45^{\circ}$ ). Dentro de los diferentes estados, la Figura 3.13(d) muestra los que corresponden a $1-100$ y $301-400$ dado que estos alcanzan el valor máximo de $\rho=1,15$ que se obtiene a partir de los valores de impedancia cuando $i=0 \mathrm{~mA}\left(\rho=\left|\Gamma_{4}+\Gamma_{5}\right|\right.$ o $\left.\rho=\left|\Gamma_{6}+\Gamma_{7}\right|\right)$. Nótese que, en el caso teórico en que la magnitud de los coeficientes de reflexión es de 1, el valor de $\rho$ corresponde a $\sqrt{2}$ y la eficiencia máxima es de $50 \%$. La Figura 3.13(d) muestra la eficiencia teórica y la eficiencia medida, ambas calculadas a partir de los valores de impedancia medidos. La pequeña diferencia entre los resultados se asocia con las pérdidas y no-linealidades en la red. La eficiencia del modulador podría incrementarse utilizando cargas más reflectivas, así como un diseño diferente de las cargas que permita cumplir la condición teórica de $\rho=2$. En el próximo capítulo se discute una alternativa más eficiente.

\subsubsection{Validación del Prototipo}

Para validar el prototipo, se diseña el sistema de control para producir una constelación 16-QAM para 8 estados de polarización diferentes, entre ellos: polarización cir- 


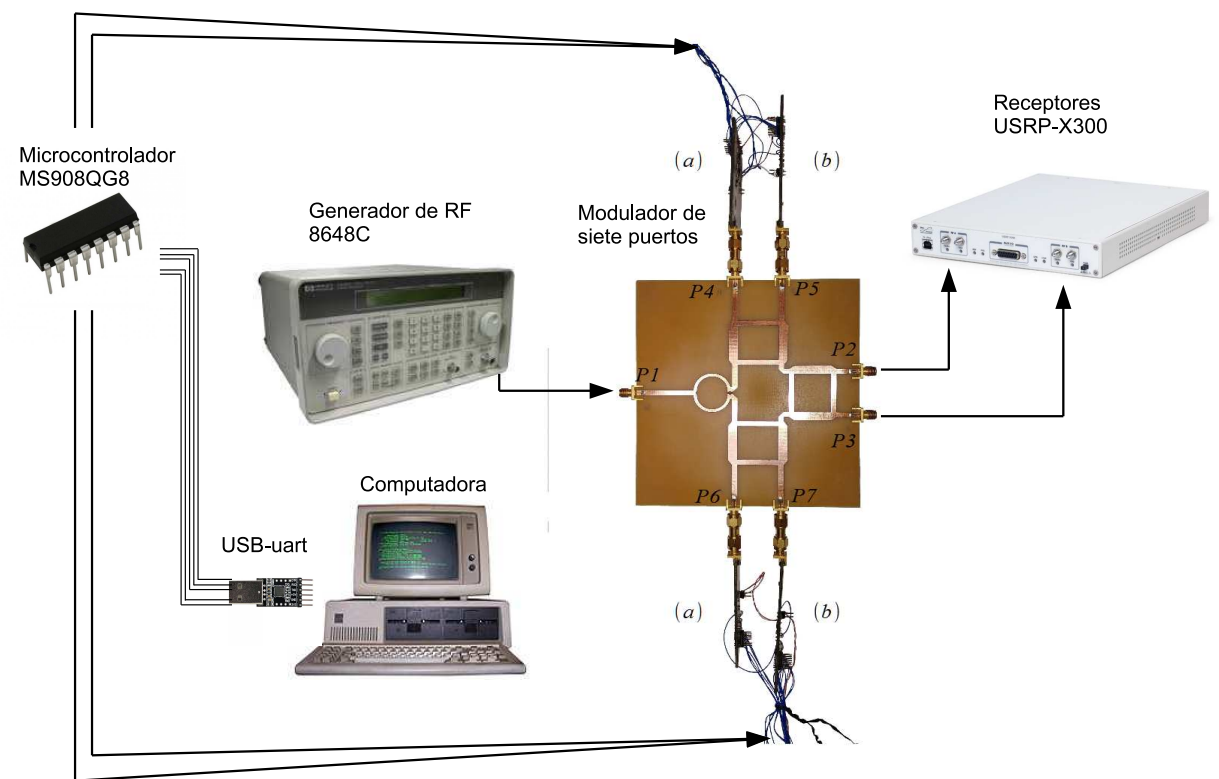

Figura 3.12: Experiencia de medición, configuración y conexión

cular derecha e izquierda, 5 estados de polarización lineal con ángulo de orientaciones $\alpha=\left\{-80^{\circ},-40^{\circ}, 0^{\circ}, 40^{\circ}, 80^{\circ}\right\}$, y un estado de polarización arbitrario $(\alpha, \beta)=\left(0^{\circ},-20^{\circ}\right)$. Utilizando estos valores, se obtiene la amplitud compleja de las señales como muestra (3.18). Los valores de corriente que configuran las cargas en los estados correspondientes, se obtienen a partir de un procedimiento de calibración, el cual se describe en el Apéndice C. $\operatorname{Los} N=128(16 \times 8)$ estados de la señal de salida se miden como muestra la Figura 3.12. En la Figura 3.3.2(a) muestra la constelación calculada como (3.18), y las Figuras 3.3.2(b) y (c) presentan los ángulos de polarización $\alpha$ y $\beta$. Como se comentó antes, cuando el ángulo de elipticidad es $\beta= \pm 45^{\circ}$, el ángulo de orientación es indeterminado.

\subsubsection{Validación con Antenas}

Para realizar una validación del modulador práctica, se llevaron a cabo mediciones utilizando las antenas de dos puertos. En consecuencia se utiliza la configuración mostrada en la Figura 3.12 pero conectando a las salidas del modulador la antena transmisora como muestra la Figura 3.11. Por otro lado, se conecta una antena receptora para captar la señal transmitida la cual se demodula utilizando el USRP-X300. Siguiendo la notación de Figura 3.1 el puerto $P_{V}$ se conecta al puerto $P_{2}$ de la red de siete puertos y $P_{H}$ se conecta a $P_{3}$ como muestra la Figura 3.3.3. La antena receptora de dos puerto se conecta al USRP-X300. La separación entre las antenas es de 2,5 metros $(>10 \lambda)$ para asegurar la condición de campo lejano. El modulador se configura utilizando los valores de corriente del Cuadro 3.1, los datos se obtienen del USRP-X300, se guardan y se procesan en MATLAB. Los ángulos de polarización se presentan en la Figura 3.13(a) y (b) en color negro, mientras que la constelación se escala (para obtener la misma escala de los resultados ideales) y se presenta en la Figura 3.13(c). Si bien esta medición no permite caracterizar ningún parámetro del modulador, funcionan como validación preliminar dado 


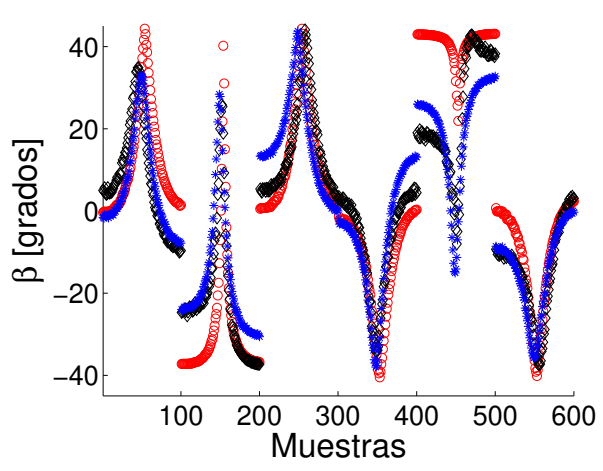

(a)

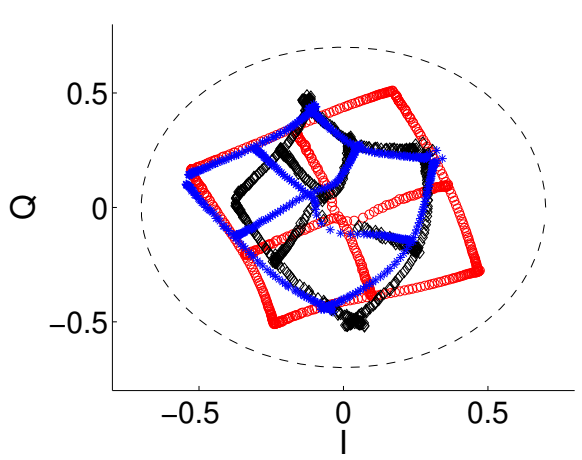

(c)

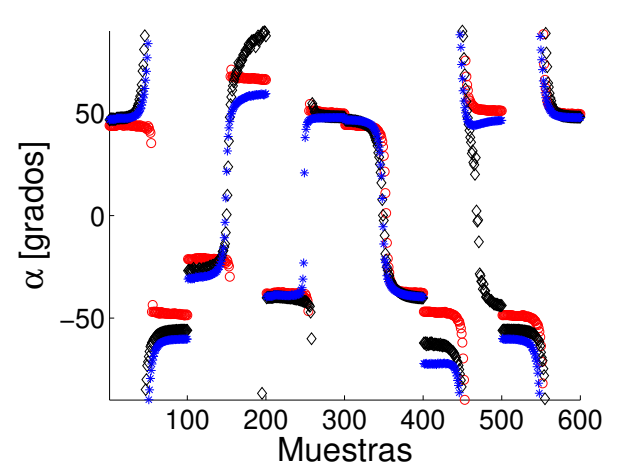

(b)

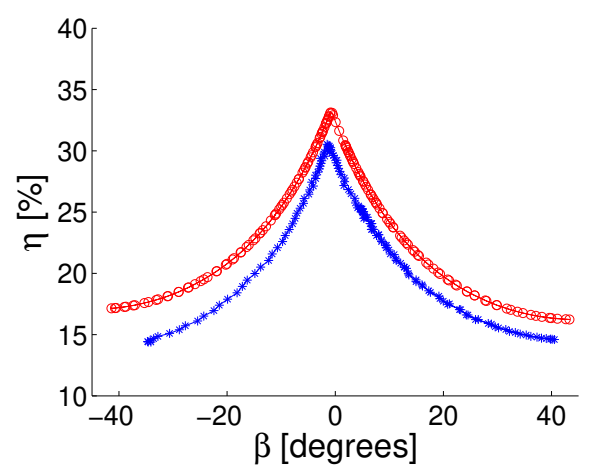

(d)

Figura 3.13: Respuesta de las salidas del modulador de siete puertos: Resultados teóricos (o rojo), resultados medidos en la salida (* azul) y resultados medidos con antenas $(\diamond$ negro). (a) Elipticidad $\beta$, (b) orientación $\alpha$, (c) envolvente compleja, (d) eficiencia.

que existe un buen acuerdo entre los resultados ideales, medidos, y los resultados con las antenas.

\subsection{Ventajas y Desventajas}

La arquitectura propuesta requiere de cuatro señales de banda base para modular la envolvente compleja de dos señales de salida. Por lo tanto, el modulador propuesto utiliza la mitad de impedancias variables y la mitad de señales de control que un sistema implementado con moduladores de seis puertos, el cual requiere de dos estructuras independientes para modular las dos salidas. Una desventaja del prototipo presentado es el bajo valor de eficiencia de conversión, $\eta \approx 32 \%$. A partir del análisis teórico de la eficiencia, se demostró que lo dispositivos de seis y siete puertos, en general presentan valores inferiores a $\eta=50 \%$. Sin embargo, se mostró que esta característica se puede mejorar utilizando un diseño diferente para las impedancias variables.

Por otro lado, las pérdidas por retorno en el modulador propuesto son altas respecto a los valores logrados con dispositivos de seis puertos. En consecuencia, en el próximo capítulo se presenta un nuevo diseño de las cargas que permiten mejorar estas características del modulador a costa de aumentar la cantidad de señales requeridas por el circuito banda base. 


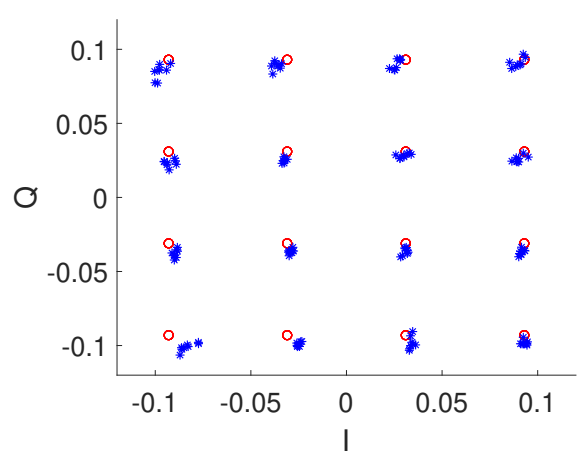

(a)

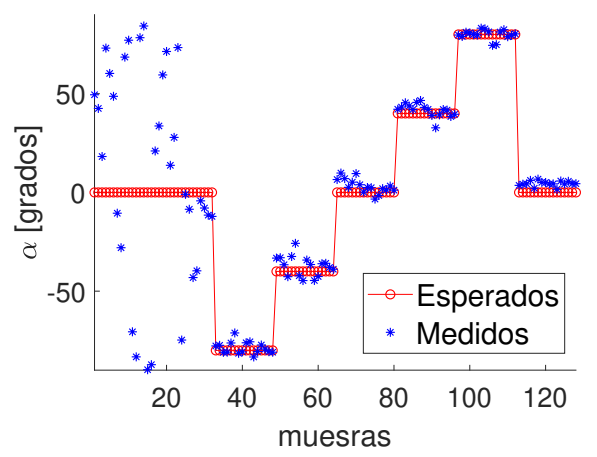

(b)

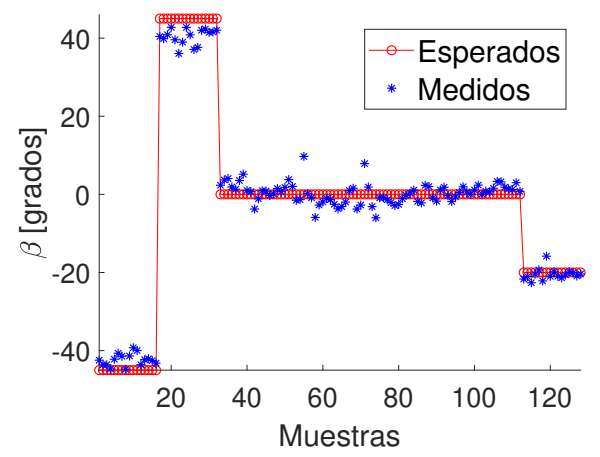

(c)

Figura 3.14: Respuesta de salida del modulador de siete puertos a 1575,42 MHz. Valores deseados (o rojo) y valores obtenidos de las mediciones (* azul) de (a) la envolvente compleja, (b) la orientación $\alpha$, y (c) la elipticidad $\beta$. 

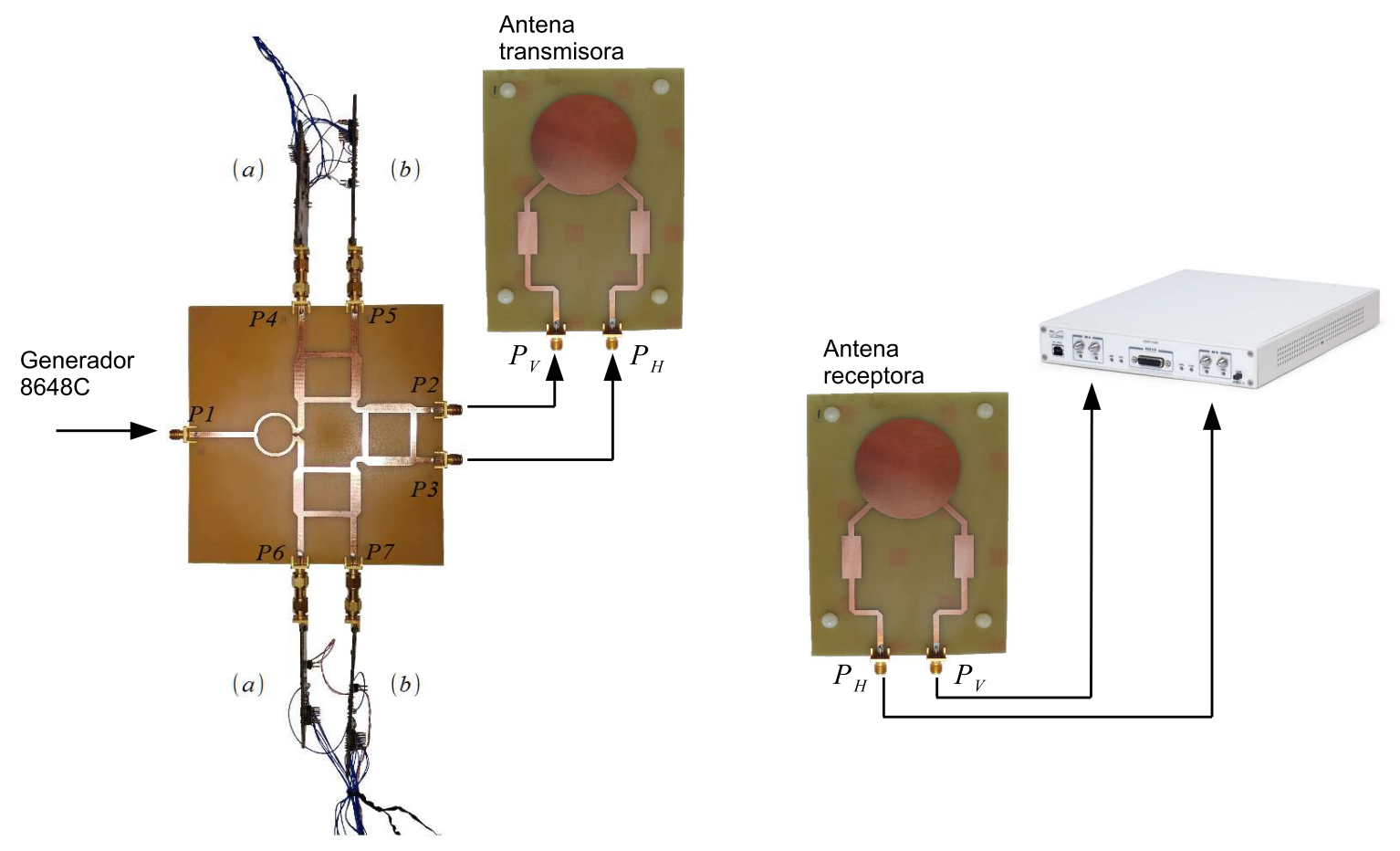

Figura 3.15: Conexión de las salidas del modulador para la medición con antenas. 


\section{Capítulo 4}

\section{Impedancias Variables}

En este capítulo se presenta el diseño de una impedancias variables propuesta para mejorar las pérdidas por retorno del modulador de siete puertos.

\subsection{Introducción}

Casi todos los sistemas inalámbricos utilizan impedancias variables para ajustar ciertos aspectos del mismo. Por ejemplo, estas se utilizan para la implementación de atenuadores [49] y moduladores [50] controlados electrónicamente, desfasadores controlados [51], redes de adaptación ajustables [52], y materiales absorbentes adaptables para radares [53]. Un enfoque común para la implementación de impedancias variables consiste en explotar la relación no lineal entre la tensión y la corriente del diodo. Por ejemplo, en [16], se construye una resistencia controlada por tensión usando un diodo Schottky polarizado en directa. Dado que el dispositivo tiene un solo grado de la libertad (la tensión de polarización), el rango de valores del coeficiente de reflexión se limita a una línea o una curva en el diagrama de Smith. Lo mismo ocurre con las impedancias variables desarrolladas en el Capítulo 3.

Existen aplicaciones en las que se requieren dispositivos capaces de controlar los valores del coeficiente de reflexión dentro de un área en el diagrama de Smith. En [54], se presenta una impedancia programable generada por un arreglo de diodos PIN. Los diodos se conectan entre si a través de líneas de transmisión. Ademas cada diodo se conecta a una fuente de corriente a través de multiplexadores. Esta alternativa utiliza 10 o más diodos para obtener coeficientes de reflexión en cualquier punto del diagrama de Smith. Debido a la cantidad de diodos y a los multiplexores, este enfoque resulta algo engorroso de implementar. Una arquitectura más simple se presenta en [55], la cual consta de cuatro varactores y un acoplador híbrido en cuadratura. El dispositivo puede controlar la impedancia dentro de un área definida por un circulo de $|\Gamma|=0,9$ en el diagrama de Smith a partir de cuatro señales de tensión. Este esquema se presenta para aplicaciones de bajos niveles de consumo de potencia debido al reducido número de diodos, sin embargo requiere de cuatro señales para controlar el valor de impedancia. Los moduladores de seis puertos y siete puertos requieren de cuatro impedancias variables para control las señales de salida, por lo tanto, este esquema resulta complejo desde el punto de vista del circuito de banda base. 


\subsection{Motivación}

\section{Aplicación en Moduladores de Siete Puertos}

Como se mencionó en el Capítulo 3, la relación entre los coeficientes de reflexión de las cargas establece las características de eficiencia y pérdidas por retorno del sistema. En particular, si se establece la relación $\Gamma_{4}=\Gamma_{5}$ y $\Gamma_{6}=\Gamma_{7}$, se minimizan las pérdidas por retorno como muestra (3.10). Sin embargo, la relación de las salidas y los coeficientes de reflexión (3.12) se reduce a

$$
\left[\begin{array}{l}
\Gamma_{4} \\
\Gamma_{6}
\end{array}\right]=\left[\begin{array}{cc}
j & -1 \\
-1 & j
\end{array}\right]\left[\begin{array}{l}
\frac{b_{2}}{a_{1}} \\
\frac{b_{3}}{a_{1}}
\end{array}\right] .
$$

Por lo tanto, para obtener un conjunto de salidas arbitrarias, cada impedancia variable por si sola, debe ser capaz de generar los valores de magnitud y fase establecidos por la ecuación anterior. A continuación, se propone una impedancia variable capaces de generar distintos valores del coeficiente de reflexión dentro de un área de cobertura en el diagrama de Smith utilizando únicamente dos diodos. Estas alternativas logran cumplir la relación $\Gamma_{4}=\Gamma_{5} \mathrm{y} \Gamma_{6}=\Gamma_{7}$ y pueden controlar la magnitud y fase de los términos $\Gamma_{4}+\Gamma_{5} \mathrm{y} \Gamma_{6}+\Gamma_{7}$ simultáneamente.

\subsection{Propuesta Basada en dos Diodos PIN}

La impedancia variable se compone de dos diodos PIN cuyo valor de impedancia se controla por la corriente de polarización directa. El diodo PIN es una excelente opción debido al rango dinámico que presenta en su valor de resistencia. En la Figura 4.1 se muestra el esquema básico del circuito donde los dos diodos se interconectan a tráves de un divisor Wilkinson. El puerto $P_{1}$ del divisor Wilkinson representa el puerto de entrada de la impedancia.

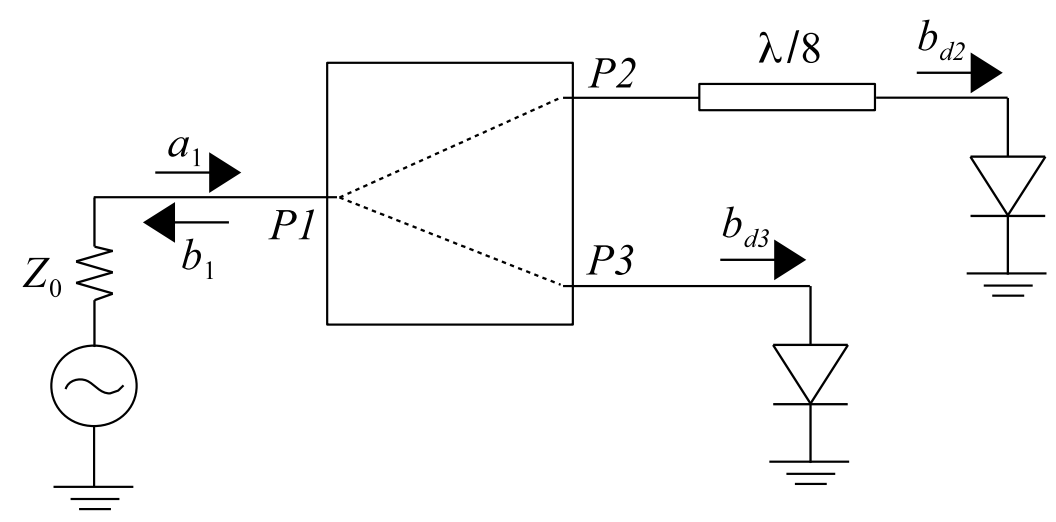

Figura 4.1: Esquema básico del circuito propuesto 


\subsubsection{Modelo Analítico del Coeficiente de Reflexión}

Este tipo de diodo tiene un comportamiento similar al diodo PN en baja frecuencia; sin embargo, en frecuencias de microondas se comporta como una resistencia variable. Como se mostró en la Sección 3.2.2, un modelo simple para representar el comportamiento del diodo cuando está polarizado en directa es

$$
Z=R(I)+j \omega L
$$

donde $\omega$ es la frecuencia angular de la señal inyectada, $L$ es la inductancia parásita debido al encapsulado, y $R$ es la resistencia de la unión, cuyo valor depende de la corriente de polarización $I$ como $R(I)=K / I^{x}$. K y $x$ son constantes de ajuste. El coeficiente de reflexión de cada diodo con respecto a la impedancia característica $Z_{0}$ resulta

$$
\Gamma_{d}(I)=\frac{R(I)+j \omega L-Z_{0}}{R(I)+j \omega L+Z_{0}}
$$

Al aplicar una señal $a_{1}$ en el puerto $P_{1}$ de el divisor Wilkinson, esta se propaga a través de la estructura hasta alcanzar la posición de los diodos donde se obtiene

$$
\begin{aligned}
& b_{d 2}=s_{21} e^{-j \phi_{2}} a_{1}, \\
& b_{d 3}=s_{31} a_{1}
\end{aligned}
$$

donde $s_{i j}$ son los parámetros del divisor de potencia (2.9) y $\phi_{2}=\pi / 4$ es el desfasaje introducido por la línea de transmisión de $\lambda / 8$. La aislación que provee la red de tres puerto evita el acoplamiento entre los puertos $P_{2}$ y $P_{3}$. Entonces, debido a la desadaptación introducida por los diodos, las señales $b_{d 2}$ y $b_{d 3}$ se reflejan hacia el divisor de potencia donde se combinan para formar la señal $b_{1}$

$$
b_{1}=s_{12} e^{-j \phi_{2}} b_{d 2} \Gamma_{d}\left(I_{2}\right)+s_{13} b_{d 3} \Gamma_{d}\left(I_{3}\right) .
$$

Los valores $I_{2}$ e $I_{3}$ son las corrientes de polarización aplicadas a cada diodo. Considerando que el divisor Wilkinson es un dispositivo recíproco $\left(s_{i j}=s_{j i}=\frac{-j}{\sqrt{2}}\right)$, entonces cuando (4.4) y (4.5) se remplazan en (4.3.1) se obtiene

$$
\begin{aligned}
b_{1} & =s_{21}^{2} e^{-j 2 \phi_{2}} \Gamma_{d}\left(I_{2}\right) a_{1}+s_{31}^{2} \Gamma_{d}\left(I_{3}\right) a_{1} \\
& =\left[j \frac{\Gamma_{d}\left(I_{2}\right)}{2}-\frac{\Gamma_{d}\left(I_{3}\right)}{2}\right] a_{1} .
\end{aligned}
$$

Finalmente, la relación entre $b_{1}$ y $a_{1}$ define el coeficiente de reflexión debido a la desadaptación entre la impedancia variable y una línea de transmisión de impedancia $Z_{0}$

$$
\Gamma_{1}\left(I_{2}, I_{3}\right)=j \frac{\Gamma_{d}\left(I_{2}\right)}{2}-\frac{\Gamma_{d}\left(I_{3}\right)}{2}=\rho\left(I_{2}, I_{3}\right) e^{j \phi\left(I_{2}, I_{3}\right)}
$$

donde $\rho$ y $\phi$ denotan la magnitud y fase del coeficiente de reflexión en el puerto $P_{1}$.

Dejando de lado la inductancia parásita $L$ del diodo, el coeficiente de reflexión $\Gamma_{d}$ es real y oscila alrededor de $(-1,1)$ cuando la corriente de polarización varía desde su valor máximo hasta cero. Por lo tanto, las corrientes $I_{2}$ y $I_{3}$ controlan la parte real e imaginaria de $\Gamma_{1}$. Idealmente, la impedancia variable propuesta alcanza un coeficiente de reflexión cuya magnitud es tan grande como $\rho=0,707$ como se muestra en la Figura 4.2(a). Sin 


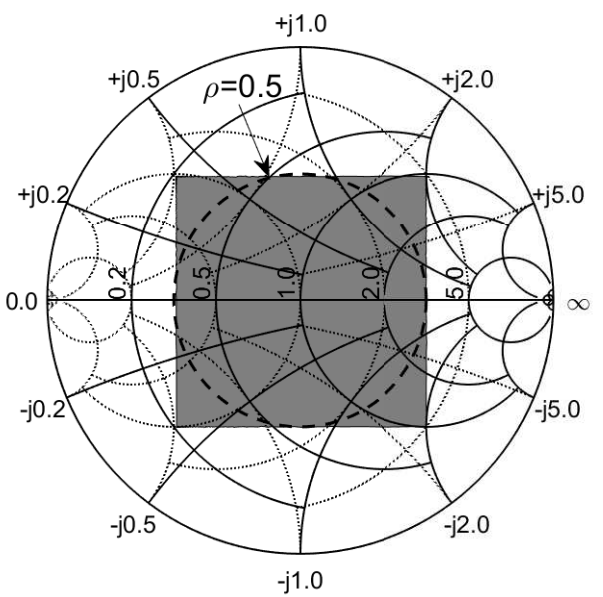

(a)

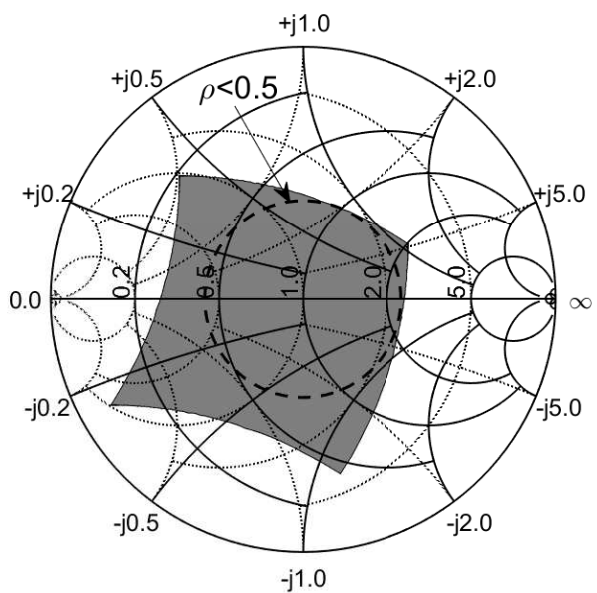

(b)

Figura 4.2: Valores de impedancia mapeados en el diagrama de Smith. Resultados simulados a partir del modelo (4.8) variando $I_{2}$ e $I_{3}$ en el rango $[0,40] \mathrm{mA}$, (a) sin considerar inductancia parásita $(L=0 \mathrm{H})$, (b) con inductancia parásita $(L=1,5 \mathrm{nH})$.

embargo, este valor máximo de $\rho$ solo es posible para un número limitado de valores de fase $\phi$. La cobertura completa del ángulo de fase $\phi$ es posible para un coeficiente de reflexión con una magnitud de $\rho \leq 0,5$.

La inductancia parásita de cada diodo desvía el área de cobertura de la impedancia variable. Por lo cual, la cobertura de fase completa se reduce a un círculo aún más pequeño, como se muestra en la línea de puntos de la Figura 4.2(b). Para corregir este problema se emplea el método de compensación presentado en la Sección 3.2.2.

\subsection{Implementación del Circuito}

Como se muestra en la Figura 4.3, las corrientes $I_{2}$ y $I_{3}$ que controlan las impedancias de los diodos. Estas se aplican sobre los ánodos, a través de los choques $L_{C H}$, mientras que los cátodos de los diodos están conectados a tierra. Los condensadores $C_{D C}$ aparecen

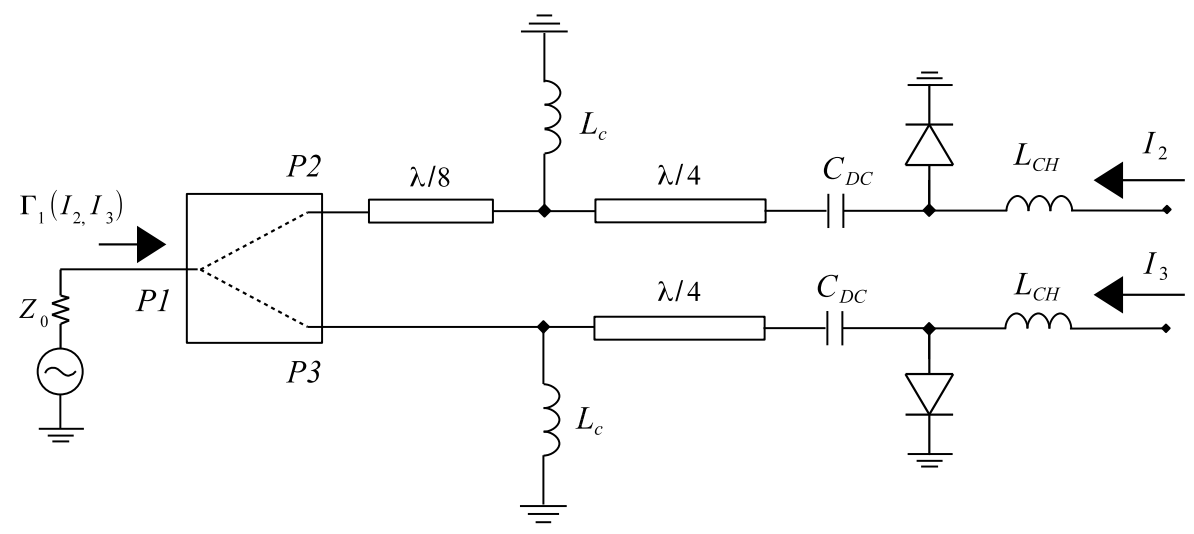

Figura 4.3: Circuito esquemático del prototipo propuesto

como un circuito abierto para las corrientes de polarización, pero como un cortocircuito 
para la señal de microondas. Las líneas de transmisión de longitud $\lambda / 4$ y los inductores $L_{c}$ compensan la inductancia parásita de los diodos. Finalmente, para generar la parte real e imaginaria del coeficiente de reflexión, la diferencia de longitud entre las dos ramas es de $\lambda / 8$. Para la implementación del prototipo se utilizan los dos circuito desarrollados para el modulador de siete puertos mostrados a la derecha en la Figura 3.4. Estos circuito corresponden a las dos ramas conectadas a los puertos $P_{2}$ y $P_{3}$ del divisor Wilkinson. Este ultimo se diseño para operar a la frecuencia 1575,42 MHz. Para obtener las dimensiones del divisor Wilkinson se simuló el circuito en el software CST microwave studio. Luego, se realizó la implementación de la red en una placa de $5 \times 5 \mathrm{~cm}$ y terminando todos los puertos con conectores SMA hembra.

\subsection{Resultados}

Primero medimos el coeficiente de reflexión en la entrada del prototipo antes de soldar los inductores de compensación. Las corrientes de polarización $I_{2}$ y $I_{3}$ fueron barridas en el rango de $[0,40] \mathrm{mA}$, tomando 20 valores espaciados logarítmicamente por fuente de corriente. El área de cobertura de la impedancia controlada propuesta sin compensar la inductancia parasitaria $L$ se representa en la Figura 4.4(a), en azul. Para este caso, la magnitud del coeficiente de reflexión puede alcanzar como máximo $\rho=0,22$ para cualquier ángulo de fase (un $\rho$ mayor sólo es posible para ángulos limitados).

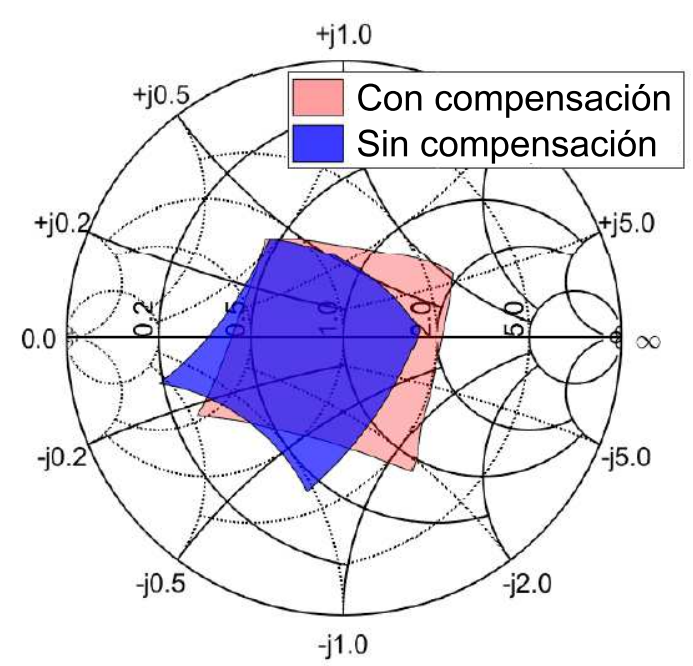

(a)

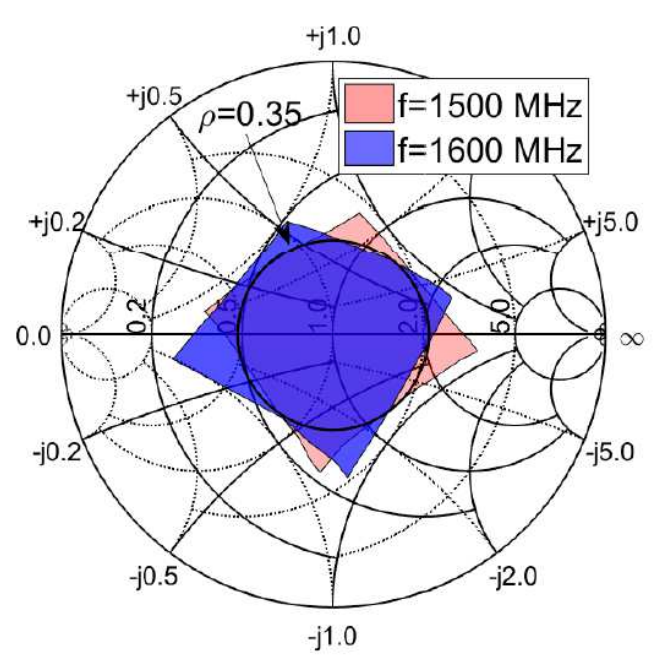

(b)

Figura 4.4: Valores de impedancia mapeados en el diagrama de Smith cuando las corrientes $I_{2}$ e $I_{3}$ varían entre $[0,40] \mathrm{mA}$. Resultados medidos (a) antes de incluir el inductor de compensación, y (b) para distintas frecuencias de operación e incluyendo la compensación.

Después de montar la compensación $L_{c}$ en el circuito, el área de cobertura de la impedancia variable se acerca más al resultado ideal, como se muestra en rosa en Figura 4.4(a). Debido a la baja estabilidad de la constante dieléctrica del FR-4, la compensación no es perfecta. Sin embargo, luego de colocar los inductores $L_{c}$ la magnitud del coeficiente de reflexión se extiende hasta $\rho=0,35$ para todo ángulo de fase.

Se midió el coeficiente de reflexión de la impedancia a diferentes frecuencias, como 
se muestra en la Figura 4.4(b). Utilizando las mismas corrientes de polarización que en la medición anterior, se muestran los coeficientes de reflexión en el rango de frecuencia de 1,5 - 1,6 GHz. Notar que la impedancia variable sigue generando un coeficiente de reflexión cuya magnitud es tan grande como $\rho=0,35$ para cualquier fase posible, ya que el área de cobertura sólo rota a medida que cambia la frecuencia.

\subsection{Conclusión}

La arquitectura consiste en dos diodos PIN conectados a un divisor Wilkinson cuya impedancia de entrada se controla a través de corrientes de polarización. La impedancia alcanza una cobertura completa de fase del coeficiente de reflexión (de 0 a $2 \pi$ ) para valores de $|\Gamma| \leq 0,35$ en el rango de 1,5 - 1,6 GHz. Estas características permiten reducir las pérdidas por retorno del modulador (3.10) sin alterar la modulación de las salidas. Sin embargo, se obtiene una baja eficiencia de conversión debido al bajo valor de $|\Gamma|$. Considerando otros diseños que logran un mayor área de cobertura en el diagrama de Smith, la principal ventaja de nuestro enfoque es que presenta el menor número de diodos (dos), lo que resulta en un bajo consumo de energía y reduce la complejidad del hardware. 


\section{Capítulo 5}

\section{Procedimiento de Calibración}

En este capítulo se presenta un procedimiento de calibración basado en un algoritmo de aprendizaje por variedades, en inglés manifold learning. El procedimiento permite controlar los valores de impedancia de cargas variables que presentan una relación no lineal con las señales de control. Se realiza la validación del método de calibración utilizando las impedancias desarrolladas en el Capítulo 4.

\subsection{Introducción}

En general, los efectos no lineales en los dispositivos electrónicos suelen dificultar la obtención de modelos analíticos representativos. Las impedancias variables son un ejemplo clásico de ello. Dependiendo de su diseño, la mayoría de las impedancias variables se controlan a través de varios parámetros no lineales. Por este motivo, se requieren de procesos de calibración capaces de recuperar los valores de los parámetros desconocidos que establecen los estados de impedancia deseados. En [54], se obtienen los parámetros de control a partir de fórmulas de síntesis, las cuales se definen del circuito específico. La mayoría de las veces, no se dispone de un modelo detallado del dispositivo debido al efecto de componentes parásitos en alta frecuencia y a la falta de información proporcionada por el fabricante a la frecuencia de interés.

En este capítulo se presenta un método para calibrar las impedancias variables basado en un algoritmo de aprendizaje por variedades conocido como "Mapa de difusión", en inglés Diffusion Maps. En lugar de utilizar un modelo predefinido de los circuitos, este método proporciona un modelado intrínseco a partir de una reparametrización de los valores de impedancia. Esta técnica fue muy utilizada por Coifman y Lafon para reducir la dimensionalidad en muchos campos de aplicación [56], y recientemente, se demostró su utilidad para modelar sistemas cuyas respuestas son una función no lineal de varios parámetros independientes [57]. El método propuesto funciona bajo el supuesto de que los valores de impedancia provienen de una caja negra controlada por una serie de parámetros independientes. El objetivo de la calibración es recuperar dichos parámetros de control desconocidos para configuran el circuito en los estados de impedancia deseados. La validación del método se realiza con un ejemplo simulado de la impedancia presentada en el Capítulo 4 y luego sobre el prototipo de impedancia variable. 


\subsubsection{Formulación del Problema}

Los moduladores de seis y siete puertos presentados anteriormente utilizan impedancias variables para generar distintos coeficientes de reflexión y así modular la señal portadora. Por lo tanto, los estados de salida de las impedancias variables se definen directamente en función del coeficiente de reflexión. El método de calibración se describe sobre la impedancia variable presentada en el capítulo anterior, cuando estas operan a una frecuencia de 1575,42 MHz. Dicho dispositivo consiste en un divisor de potencia de Wilkinson que conecta dos diodos PIN a través de líneas de transmisión de diferentes longitudes eléctricas. Cada diodo se polariza mediante una corriente de polarización que provoca un cambio (no lineal) en su resistencia. En consecuencia, el valor complejo del coeficiente de reflexión se establece a través de una combinación de dos parámetros de control como muestra (4.8). Dichos parámetros son las corrientes de polarización de los diodos.

En este capítulo se utiliza la siguiente formulación. El conjunto $\bar{\Gamma}=\left\{\bar{\gamma}^{i}\right\}_{i=1}^{m}$ consiste de $m$ valores del coeficiente de reflexión de la impedancia variable generados con los valores de los parámetros de control $\bar{\Theta}=\left\{\bar{\theta}^{i}\right\}_{i=1}^{m}$. Cada valor de $\bar{\theta}^{i}$ es un vector de $p \times 1(p=2)$. Además, el conjunto $\left\{\theta^{i_{j}}\right\}_{j=1}^{L}$ define $L$ valores adicionales que corresponden a pequeñas perturbaciones alrededor de $\bar{\theta}^{i}$. Los coeficientes de reflexión generados por estos parámetros adicionales forman una nube de puntos alrededor del coeficiente $\bar{\gamma}^{i}$. Se considera que el conjunto de valores $\bar{\Gamma}$ y sus correspondientes valores adicionales son datos de entrenamiento que se encuentran disponibles previamente. Finalmente, el conjunto $\Gamma=\left\{\gamma^{i}\right\}_{i=1}^{M}$ contiene los $M$ valores deseados del coeficiente de reflexión y $\Theta=\left\{\theta^{i}\right\}_{i=1}^{M}$ es el conjunto de los respectivos parámetros. El objetivo de la calibración es obtener los parámetros $\Theta$ a partir del conjunto $\Gamma$.

\subsection{Descripción del Método de Mapa de Difusión}

El método de calibración que se construye en esta sección surge del algoritmo de mapa de difusión propuesto por Coifman y Singer en [57]. En dicho algoritmo, primero se construye un kernel o función núcleo para captar el comportamiento de un conjunto de observaciones de entrenamiento. Luego, se realiza una descomposición en autovalores y autovectores para mapear las observaciones al nuevo espacio de parámetros. El kernel utilizado se denomina kernel de difusión y se optimiza de forma iterativa.

\subsubsection{Kernel de Difusión}

Siguiendo [57], el kernel se calcula a partir de una aproximación de segundo orden de la distancia entre parámetros, dada por

$$
\left\|\theta^{i}-\theta^{j}\right\|^{2} \approx 2\left(\gamma^{i}-\gamma^{j}\right)^{T}\left[C_{\gamma^{i}}^{-1}+C_{\gamma^{j}}^{-1}\right]\left(\gamma^{i}-\gamma^{j}\right)
$$

donde $C_{\gamma^{i}}$ es una covarianza y representa la desviación alrededor de una observación $\gamma^{i}$ debido a pequeñas perturbaciones de $\theta^{i}$. Esta información adicional está disponible a partir de las perturbaciones de los parámetros de control en $\bar{\Theta}$. Por lo tanto, podemos 
estimar empíricamente la matriz de covarianza para las observaciones de entrenamiento en $\bar{\Gamma}$ como

$$
C_{\bar{\gamma}^{i}}=\frac{1}{L} \sum_{j=1}^{L}\left(\bar{\gamma}^{i_{j}}-\mu_{i}\right)\left(\bar{\gamma}^{i_{j}}-\mu_{i}\right)^{T}
$$

donde $\mu_{i}=\frac{1}{L} \sum_{j=1}^{L} \bar{\gamma}^{i_{j}}$, y $\left\{\bar{\gamma}^{i_{j}}\right\}_{j=1}^{L}$ es una nube de $L$ puntos alrededor de $\bar{\gamma}^{i}$ dada por el conjunto de valores $\left\{\theta^{i_{j}}\right\}$. A partir de (5.1) se construye una matriz de afinidad $A$ de $M \times m$ que mide la cercanía entre observaciones en $\bar{\Gamma}$ y los valores deseados en $\Gamma$. Dicha afinidad se basa en un kernel Gauseano el cual depende de un parámetros de escala $\varepsilon$ como sigue

$$
A_{j i}=\exp \left\{-\frac{\left\|C_{\bar{\gamma}^{i}}^{-1}\left(\bar{\gamma}^{i}-\gamma^{j}\right)\right\|^{2}}{\varepsilon}\right\} .
$$

Los índices $j$ e $i$ denotan la fila y la columna de la matriz $A$, respectivamente. A partir de $A$ se calcula la matriz del kernel de difusión $W$, que representa la afinidad entre las observaciones de entrenamiento en $\bar{\Gamma}$

$$
W=S^{-\frac{1}{2}} A^{T} A S^{-\frac{1}{2}}
$$

donde $S$ es una matriz diagonal que contiene la suma de cada fila de $A^{T} A$. Asimismo, $W$ se puede obtener de la siguiente manera

$$
W_{j i}=\frac{\pi}{\sqrt{\operatorname{det}\left(C_{\tilde{\gamma}}\right)}} \times \exp \left\{-\frac{\left(\bar{\gamma}^{i}-\bar{\gamma}^{j}\right)^{T}\left[C_{\bar{\gamma}^{i}}^{-1}+C_{\bar{\gamma}^{j}}^{-1}\right]\left(\bar{\gamma}^{i}-\bar{\gamma}^{j}\right)}{\varepsilon}\right\}
$$

donde la expresión $\operatorname{det}\left(C_{\tilde{\gamma}}\right)$ es el determinante de la matriz de covarianza $(5.2)$ y $\tilde{\gamma}=$ $\left(\bar{\gamma}^{i}+\bar{\gamma}^{j}\right) / 2$.

\subsubsection{Descomposición}

La descomposición en autovalores y autovectores se realiza utilizando la matriz normalizada $\tilde{A}=A S^{-\frac{1}{2}}$. Los valores $\left\{\lambda_{i}\right\}_{i=1}^{m},\left\{\varphi_{i}\right\}_{i=1}^{m}, \mathrm{y}\left\{\psi_{i}\right\}_{i=1}^{M}$ definen los autovalores, los autovectores a izquierda y a derecha de la matriz $\tilde{A}$, respectivamente. Notar que $\left\{\lambda_{i}^{2}\right\}$ y $\left\{\varphi_{i}\right\}$ son los autovalores y autovectores de la matriz (5.4), y establecen la reparametrización de las observaciones de entrenamiento $\bar{\Gamma}$. En consecuencia, el mapeo de $\bar{\Gamma}$ en un espacio de parámetros formado por lo autovalores de $W$ está dado por

$$
\Phi_{d}: \bar{\gamma}^{i} \rightarrow\left[\lambda_{1}^{2} \varphi_{1}\left(\bar{\gamma}^{i}\right), \ldots, \lambda_{d}^{2} \varphi_{d}\left(\bar{\gamma}^{i}\right)\right]^{T} .
$$

La dimensión del espacio, $d$, se establece considerando los autovectores correspondientes a los autovalores de mayor tamaño. Asimismo, $\left\{\psi_{i}\right\}$ son los autovectores de $\tilde{A} \tilde{A}^{T}$ y establecen la reparametrización de los valores $\Gamma$, los cuales se calculan como $\varphi_{i}$

$$
\psi_{i}=\frac{1}{\lambda_{i}} \tilde{A} \varphi_{i}
$$

La función que mapea las observaciones en $\Gamma$ a la reparametrización formada por los $d$ autovectores ordenados, es

$$
\Psi_{d}: \gamma^{i} \rightarrow\left[\psi_{1}\left(\gamma^{i}\right), \ldots, \psi_{d}\left(\gamma^{i}\right)\right]^{T}
$$


Para obtener los parámetros desconocidos en $\Theta$ se calcula la siguiente suma pesada de los parámetros de entrenamiento $\bar{\Theta}$

$$
\hat{\theta}^{i}=\sum_{j: \Psi\left(\bar{\gamma}^{i}\right) \in \mathcal{N}_{i}} \rho_{j}\left(\gamma^{i}\right) \bar{\theta}^{j}
$$

donde $\mathcal{N}_{i}$ consta de los $k$ vecinos mas cercanos de $\Psi\left(\gamma^{i}\right)$ entre los valores de entrenamiento, y $\rho_{j}\left(\gamma^{i}\right)$ son los coeficientes de la interpolación lineal que surgen de resolver el siguiente sistema lineal

$$
\left\{\bar{\theta}^{j}\right\}_{j: \Psi\left(\bar{\gamma}^{i}\right) \in \mathcal{N}_{i}}=\left\{\rho_{j}\left(\gamma^{i}\right) \Psi_{d}\left\{\bar{\gamma}^{j}\right\}\right\}_{j: \Psi\left(\bar{\gamma}^{i}\right) \in \mathcal{N}_{i}} .
$$

\subsubsection{Parámetro de Escala}

El parámetro de escala $\varepsilon$ se relaciona con una medida de similaridad entre puntos del espacio de observaciones. El cálculo de la matriz de afinidad (5.3) y el desempeño del algoritmo son sensibles a el valor de $\varepsilon$. Para encontrar el valor óptimo del parámetro, se define un error de validación la cual se minimiza en función del mismo.

En primer lugar, siguiendo [57] se define la función $\Phi^{-1}$ que mapea los parámetros de entrenamiento al espacio de observaciones, lo cual aproxima a las observaciones de entrenamiento en $\bar{\Gamma}$.

$$
\Phi^{-1}(\bar{\theta})=\sum_{i: \bar{\theta}^{i} \in \mathcal{B}_{\theta}} \beta_{i}(\bar{\theta}) \bar{\gamma}^{i}
$$

donde $\mathcal{B}_{\theta}$ es un conjunto de vecinos alrededor de $\bar{\theta}$, y $\beta_{i}$ son los coeficientes de interpolación dados por

$$
\beta_{i}(\bar{\theta})=\frac{\exp \left(\frac{-\left\|\bar{\theta}-\bar{\theta}^{i}\right\|^{2}}{\sigma_{\beta_{i}}}\right)}{\sum_{i: \bar{\theta}^{i} \in \mathcal{B}_{\theta}} \exp \left(\frac{-\left\|\bar{\theta}-\bar{\theta}^{i}\right\|^{2}}{\sigma_{\beta_{i}}}\right)}
$$

donde $\sigma_{\beta_{i}}$ define la mínima distancia entre $\bar{\theta}^{i}$ y sus vecinos mas cercanos. Finalmente, el error de validación se define como

$$
E_{V}\left(\bar{\gamma}^{i}\right)=\left\|\bar{\gamma}^{i}-\Phi^{-1}\left(\bar{\theta}^{i}\right)\right\|^{2}
$$

Este error mide la precisión de $\Phi^{-1}$ como estimador de $\bar{\gamma}^{i}$. Se obtiene el error de validación medio calculando el promedio de (5.13) para todas las observaciones de entrenamiento. Luego de este procedimiento se obtiene el valor óptimo del parámetro de escala, el cual minimiza el error medio. Este procedimiento se describe en la próxima sección como etapa de entrenamiento.

\subsection{Implementación del Método}

El método de calibración propuesto se emplea en dos etapas: primero, una etapa de entrenamiento basada en la optimización de la escala del kernel gaussiano $\varepsilon$; y segundo, una etapa de estimación en la que se recuperan los parámetros de control desconocidos en $\Theta$. 


\section{Etapa de Entrenamiento:}

1. Obtener $m$ observaciones de entrenamiento correspondientes a los parámetros $\bar{\Theta}$.

2. Generar nubes de observaciones adicionales correspondientes a las perturbaciones de los parámetros de entrenamiento, y calcular la covarianza local $C$ de cada observación de entrenamiento como (5.2).

3. Calcular la matriz de Kernel $W$ a partir de (5.5), utilizando un valor arbitrario para el parámetro $\varepsilon$.

4. Calcular la descomposición de $W$ obteniendo los autovalores $\left\{\lambda_{i}^{2}\right\}$ y autovectores $\left\{\varphi_{i}\right\}$.

5. Construir el mapa $\Phi_{d}$ de acuerdo con (5.6) a partir de los autovalores y autovectores de $W$.

6. Construir el mapa inverso $\Phi_{d}^{-1}$ con (5.11).

7. Repetir los pasos 3 - 6 para diferentes valores de $\varepsilon$, hasta encontrar el óptimo el cual minimiza el error de validación medio (5.13).

\section{Etapa de Estimación:}

1. Dados los valores deseados $\Gamma$, calcular la matriz de afinidad normalizada $\tilde{A}$ como indica (5.3) utilizando el valor óptimo de $\varepsilon$.

2. Calcular $\psi_{i}$ como la combinación pesada de $\varphi_{i}$ de acuerdo con (5.7).

3. Construir el mapa $\Psi_{d}$ dado por (5.8), para obtener la reparametrización de los parámetros de control.

4. Obtener los parámetros desconocidos siguiendo (5.9).

\subsubsection{Ejemplo Simulado}

Siguiendo la descripción de la impedancia variable presentada en la Sección 4.3, se construye un modelo para sintetizar las observaciones del coeficiente de reflexión de la carga en función de las corrientes de polarización, las cuales representan los parámetros de control. Al variar los valores de corriente $\left(I_{2}, I_{3}\right)$ en el rango de $[0,40] \mathrm{mA}$, se obtiene un área de cobertura en el diagrama de Smith como se muestra en la Figura 5.1. Los valores en el diagrama están normalizados a través de la impedancia característica $Z_{0}=50 \Omega$. Cabe mencionar que el modelo utilizado para la simulación es una simplificación de la respuesta real de la carga, debido a que sus parámetros se consideran constantes e independientes de la frecuencia y de las corrientes de polarización, y tales suposiciones no son ciertas en la práctica.

Con la intensión de calibrar este dispositivo simulado, seleccionamos los parámetros de entrenamiento $\bar{\Theta}$ para generar las observaciones $\bar{\Gamma}$ de la siguiente manera: ambas corrientes se barren en el rango de $[0,40] \mathrm{mA}$, tomando 20 valores espaciados logarítmicamente para cada fuente de corriente. La combinatoria entre los valores de corrientes 


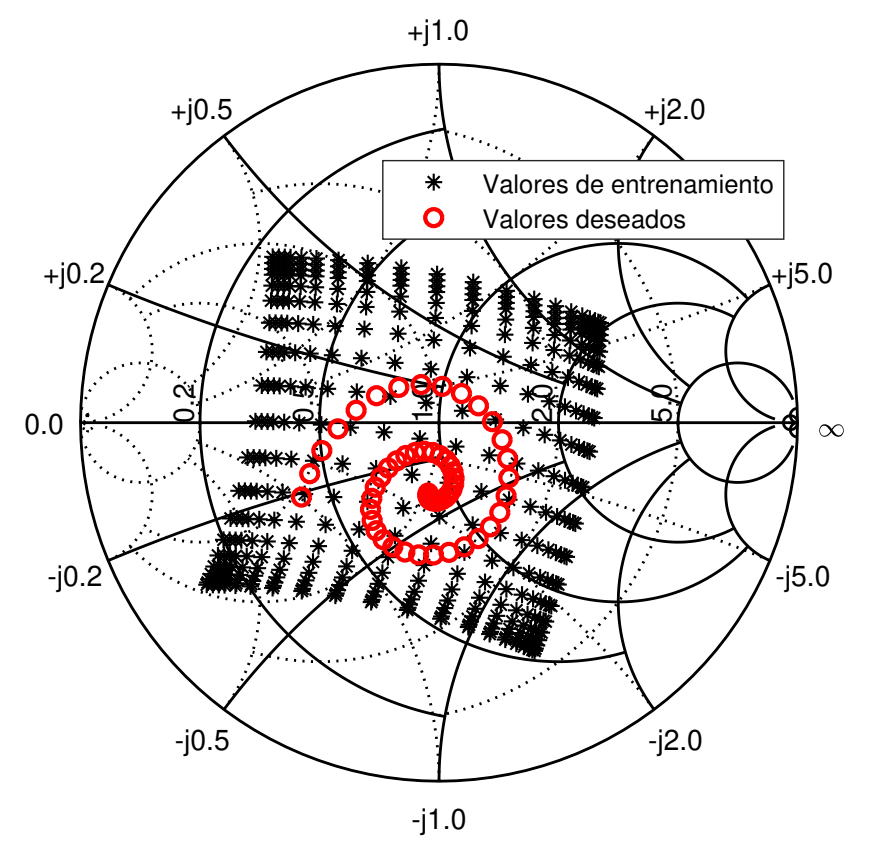

Figura 5.1: Valores simulados del coeficiente de reflexión (4.7) mapeados en el diagrama de Smith. Resultados de los datos de entrenamiento y los valores deseados.

produce $m=400$ valores de impedancia distintos. Asimismo, los coeficientes de reflexión deseados se seleccionan para formar una espiral en el gráfico de Smith. La Figura 5.1 muestra los valores de entrenamiento (asterisco negro) y los valores deseados (circulo rojo).

Siguiendo los pasos del método, cada parámetro de entrenamiento en $\bar{\Theta}$ se utiliza para generar los parámetros adicionales $\left\{\theta^{i_{j}}\right\}$, obteniendo las nubes alrededor de las observaciones en $\bar{\Gamma}$. Específicamente, se generan $L=20$ valores generados con una distribución Gaussiana alrededor de los valores de corriente $\left\{\bar{\theta}_{1}^{i}\right\}$ and $\left\{\bar{\theta}_{2}^{i}\right\}$ de la siguiente manera

$$
\begin{aligned}
& \theta_{1}^{i_{j}}=\bar{\theta}_{1}^{i}+n_{j}\left(\bar{\theta}_{1}^{i}\right) \\
& \theta_{2}^{i_{j}}=\bar{\theta}_{2}^{i}+n_{j}\left(\bar{\theta}_{2}^{i}\right)
\end{aligned}
$$

donde $n_{j}\left(\bar{\theta}^{i}\right) \sim \mathcal{N}\left(0, \bar{\theta}^{i} k\right)$ es una variable aleatoria y $k \in \mathbb{R}$ es una constante. Luego, utilizando ambos conjuntos $\left\{\theta_{1}^{i_{j}}\right\}_{j=1}^{L}$ y $\left\{\theta_{2}^{i_{j}}\right\}_{j=1}^{L}$ se genera la nube de puntos alrededor de la observación $\bar{\gamma}^{i}$. La varianza de la distribución de $n_{j}\left(\bar{\theta}^{i}\right)$ depende del valor $\bar{\theta}^{i}$, de esta manera, se logra que las nubes alrededor de cada observación de entrenamiento sean de similar tamaño. Por lo tanto, se explora la no linealidad entre los parámetros y las observaciones alrededor de toda el área de cobertura.

Siguiendo los pasos correspondientes a la etapa de entrenamiento del método, se repite el paso 2 para diferentes constantes $k$ y el paso 3 para diferentes valores del parámetros de escala. Se obtiene el error de validación medio (5.13) promediando sobre todas las observaciones de entrenamiento en función de $k$ y $\varepsilon$, como se muestra en la Figura 5.2. Se destacan los siguientes puntos: al aumentar el valor de $k$, el valor mínimo del error de validación medio ocurre en un valor de $\varepsilon$ menor, y viceversa. Por otro lado, para valores de $k$ mayores a 0,27 , el valor de error mínimo comienza a aumentar respecto a los otros 


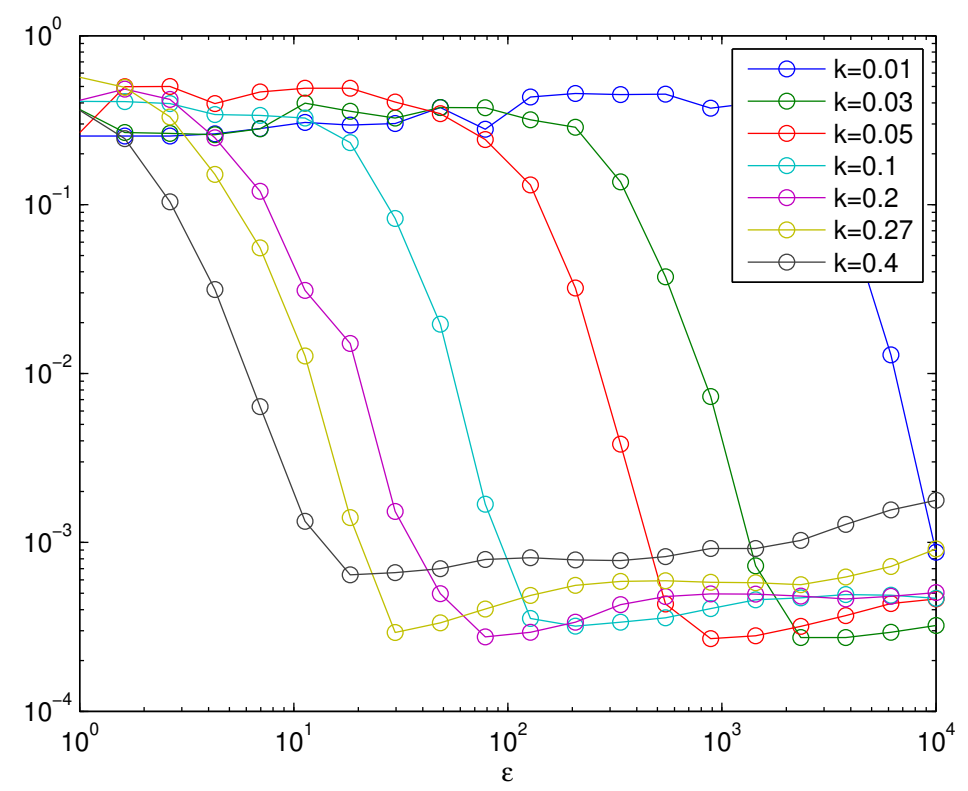

Figura 5.2: Error medio de validación obtenido a partir de observaciones de entrenamiento en función de la escala $\varepsilon$, a partir de diferentes valores de la constante de varianza $k$.

casos. Se elige un valor intermedio de $k=0,05$, el cual obtiene el error mínimo, en la escala $\varepsilon=1000$ aproximadamente.

Comenzando la etapa de estimación, se recuperan los valores de las corrientes correspondientes a los estados de impedancia deseados. Así, a partir de la matriz normalizada $\tilde{A}$, calculada con los valores óptimos de los parámetros $k$ y $\varepsilon$, se construye la función de mapeo $\Psi_{2}$ dada por (5.7). Para obtener los parámetros deseados en $\Theta$, se calcula (5.9). Los valores estimados de las corrientes $\hat{\theta}$ se utilizan para en el modelo simulado para obtener los coeficientes de reflexión. De esta manera se cuantifica la precisión del método a partir del siguiente error de estimación

$$
E_{M}=\frac{1}{M} \sum_{i=1}^{M} \frac{\left\|\gamma_{e}^{i}-\gamma^{i}\right\|}{\left\|\gamma^{i}\right\|}
$$

donde $\gamma_{e}$ es la estimación del coeficiente de reflexión deseado $\gamma$. En este caso, el error de estimación (5.16) resulta $E_{M}=0,038 \%$, mostrando una gran coincidencia entre los puntos en la gráfica de Smith, como se muestra en la Figura 5.3.

\subsubsection{Ejemplo Medido}

En esta sección se considera el prototipo de la impedancia variable presentado en el Capítulo 4 implementada a una frecuencia de 1575,42 MHz. Este prototipo es una terminación de impedancia controlada por dos fuentes digitales de corriente cuyos valores pueden variar entre $[0,40] \mathrm{mA}$ con una resolución de $\Delta I=9,76 \mu \mathrm{A}$. Para establecer los estados de impedancia de la carga, una computadora controla las fuentes transmitiendo palabras de 12-bit a través de una interfaz periférica serie (SPI). De esta manera, las palabras digitales generan valores de corriente cuantificados. En este trabajo, se asume que los parámetros estimados en (5.9) toman cualquier valor entre $[0,40] \mathrm{mA}$ porque los 


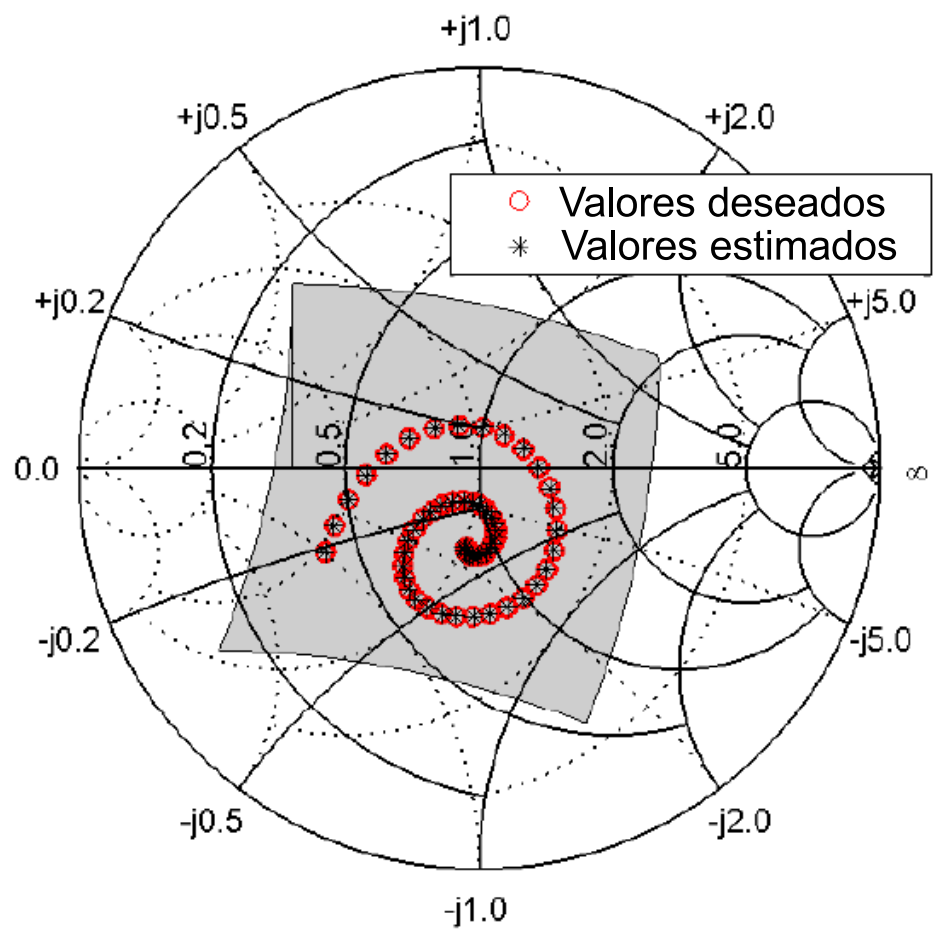

Figura 5.3: Respuesta simulada de la impedancia variable mapeada en el gráfico de Smith. Observaciones deseadas y observaciones obtenidas a partir de la simulación.

parámetros en (5.8) no tienen en cuenta la información de cuantización de las corrientes. Este problema se reflejará en el error de estimación de los resultados. Para implementar el método de calibración sobre el prototipo se toman los mismos parámetros de entrenamiento definidos en el ejemplo simulado. Cada parámetro de entrenamiento $\bar{\theta}^{i}$ se utiliza para generar las $L=20$ perturbaciones dadas por (5.16) y utilizando $k=0,05$. Por lo tanto, se midió la respuesta de impedancia a estos valores de corriente y se obtuvieron las $m=400$ observaciones de entrenamiento y las correspondientes nubes de puntos. Luego, se definió el conjunto de valores de impedancia deseados, el cual forman una espiral en el gráfico de Smith. La Figura 5.4 muestra los coeficientes de reflexión de entrenamiento junto con la espiral deseada, todos los valores se calculan con una impedancia característica $Z_{0}=50 \Omega$.

Una vez medidos todos los datos de entrenamiento, se repiten los pasos $3-7$ de la etapa de entrenamiento para diferentes valores de la escala $\varepsilon$. El valor óptimo de la escala corresponde al valor mínimo del error medio de validación. La Figura 5.5 muestra el error de validación medio en función de la escala $\varepsilon$ donde se destaca el valor óptimo de $\varepsilon$ alrededor de 1000 .

Para la etapa de estimación se obtiene el mapeo $\Psi_{2}$, se encuentran las corrientes de control deseadas (5.9), y se mide la respuesta del prototipo configurado con estos valores. El error de estimación (5.16) resulta $E_{M}=7 \%$. Las observaciones estimadas y las deseadas se muestran en la Figura 5.6, donde se observa una gran coincidencia entre ellas. 


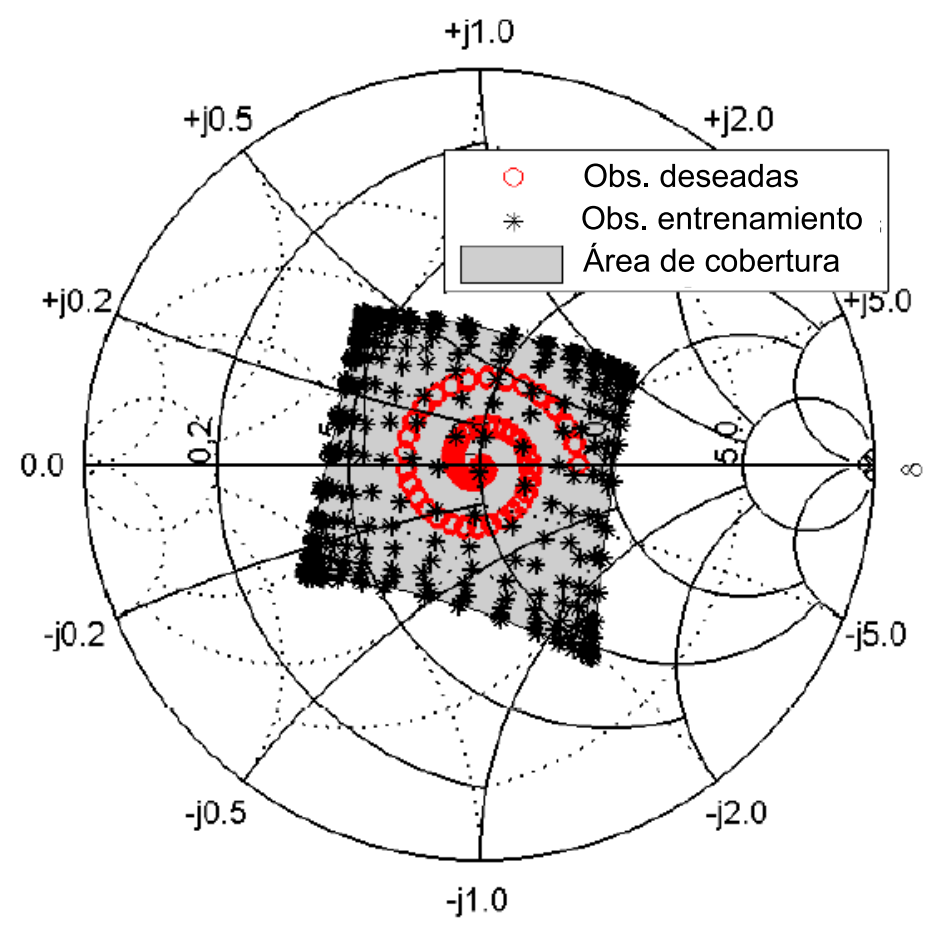

Figura 5.4: Respuesta medida de la impedancia variable del Capítulo 4 a 1575,42 MHz. Observaciones de deseadas y observaciones obtenidas de las mediciones.

\subsection{Conclusión}

En este trabajo, se propuso un método para calibrar las impedancias variables no lineales basado en la técnica de aprendizaje por variedades (manifold learning). Este enfoque de calibración se basa en un algoritmo supervisado que aprende el modelo intrínseco de la carga a través de un conjunto de datos de entrenamiento, y no requiere del modelo analítico del dispositivo cuyos parámetros son un tanto inciertos. Partiendo del supuesto de que las impedancias variables están controladas por varios parámetros independientes, el objetivo del método es la recuperación de esos parámetros de control para establecer un conjunto de valores de impedancia deseados a la frecuencia de funcionamiento. Se validó el método utilizando datos sintéticos y datos reales. El prototipo utilizado consiste en la impedancia variable presentada en [18], que consiste en una impedancia de valor complejo controlada por dos fuentes de corriente que polarizan dos diodos PIN en los puertos de un divisor Wilkinson. Para ambos ejemplos, se demuestra que se puede recuperar con precisión las corrientes de control deseadas de la carga, incluso en el ejemplo práctico que resulta difícil modelar la respuesta de la carga.

Para futuras investigaciones se planea extender el procedimiento de calibración al dominio de la frecuencia. Nuestro objetivo es determinar la corriente de polarización de control que ajusta la carga variable para producir una impedancia deseada a diferentes frecuencias de funcionamiento. Por lo tanto, los datos de entrenamiento deben incluir observaciones de los coeficientes de reflexión a diferentes frecuencias. 


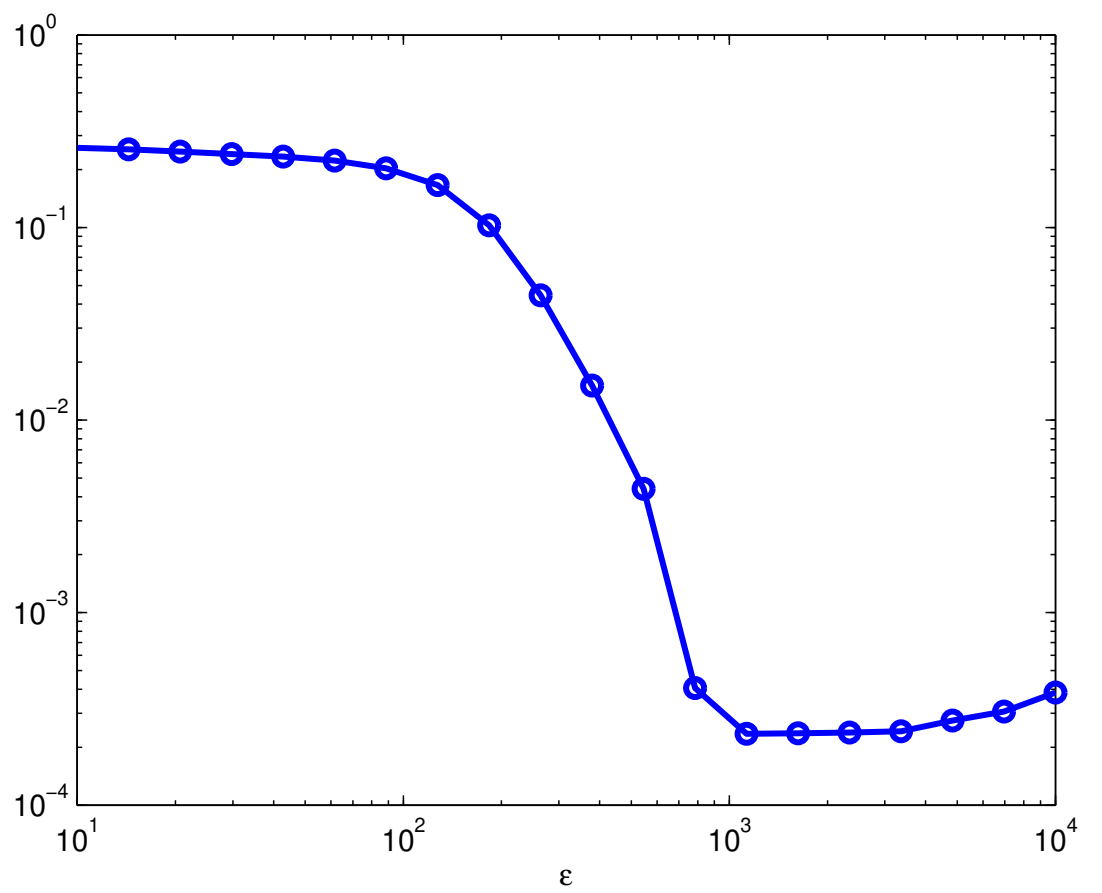

Figura 5.5: Error medio de validación en función de la escala $\varepsilon$ del kernel gaussiano calculado para todos los datos de entrenamiento del prototipo.

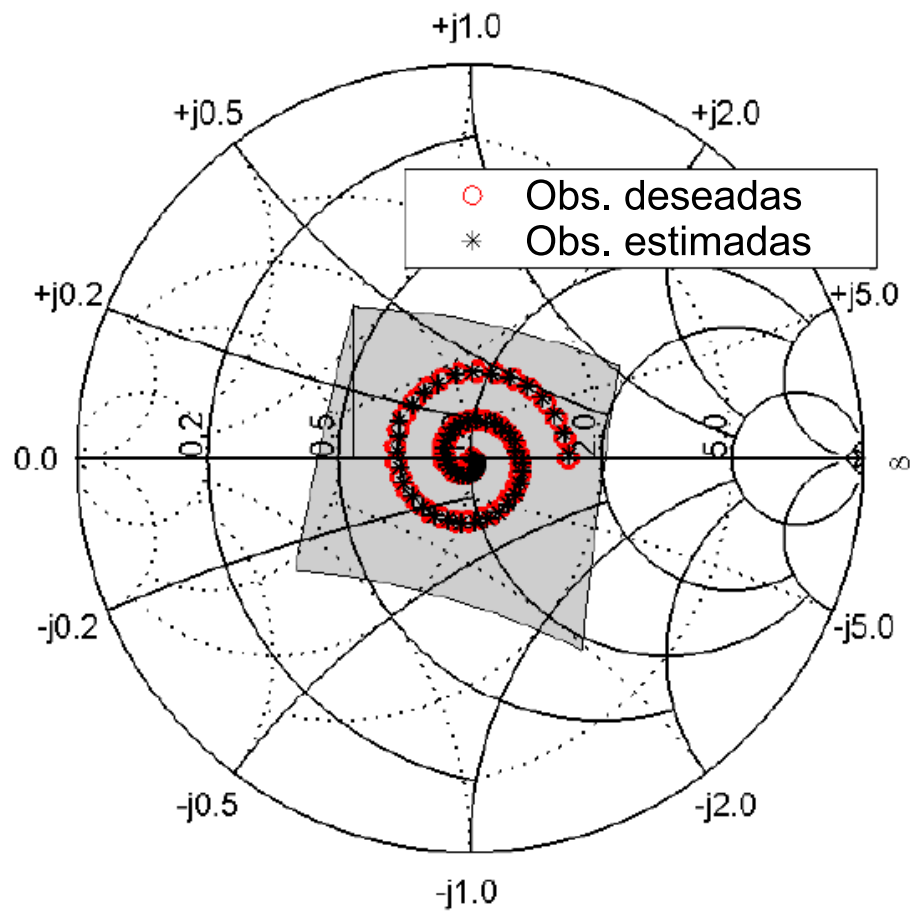

Figura 5.6: Respuesta medida del prototipo de la impedancia variable mapeada en el gráfico de Smith. Observaciones deseadas y observaciones obtenidas a partir de las mediciones. 


\section{Capítulo 6}

\section{Demodulador de Multiples Puertos}

En este capítulo se presentan los fundamentos teóricos y el diseño de demoduladores de múltiples puertos para aplicaciones en arreglos de sensores. El esquema propuestos es una variante del demodulador de seis puertos presentado en el Capítulo 2. Se desarrollan las expresiones analíticas para arquitecturas de $N$ puertos y un nuevo esquema de demodulación basado en la transformación de Hilbert. Para validar los conceptos teóricos, se desarrollan dos prototipos basados en una estructura de siete puertos. Uno de ellos es un demodulador capaz de recuperar las envolventes complejas de las señales recibidas por dos antenas a partir de cuatro mediciones de potencia, y el otro prototipo permite demodular tres envolventes complejas provenientes de tres antenas diferentes a partir de tres mediciones de potencia.

\subsection{Introducción}

La utilización de arreglos de sensores en la tecnología inalámbrica permite incorporar funciones adicionales al sistema, como la medición del ángulo de arribo y la polarización. En general, un arreglo de sensores consiste en varias antenas cada una asociada a un receptor heterodino u homodino en cuadratura, compartiendo un oscilador local. La implementación de estos dispositivos considerando las arquitecturas convencionales resulta muy compleja en términos de cantidad de componentes y consumo de potencia, especialmente cuando se requiere una función adicional de detección de ángulo de arribo o polarización además de la función de comunicaciones [58, 59]. Una alternativa mas conveniente para diseñar dispositivos de bajo costo y consumo de energía consiste en utilizar varios receptores de seis puertos conectados al mismo oscilador local [13].

En este capítulo se plantea un esquema diferente para la implementación de arreglos de sensores. En lugar de utilizar múltiples receptores independientes, se propone una única arquitectura de múltiples puertos capaz de demodular las señales a partir de mediciones de potencia. Para simplificar el circuito se plantea un esquema de demodulación basado en la transformada de Hilbert. La principal ventaja del sistema propuesto respecto a los receptores de seis puertos es la reducción del número de detectores de potencia utilizados, alcanzando un circuito mas simple, de menor consumo de energía. Como consecuencia del diseño, se incorporan operaciones adicionales al algoritmo de procesamiento digital. A continuación, se extienden los fundamentos teóricos de seis puertos para el diseño de receptores de $N$ puertos y se presentan los esquemas de demodulación propuestos. 
Finalmente, se validan lo conceptos teóricos a partir de dos prototipos de siete puertos implementados para operar a una frecuencia de 1575,42 MHz.

\subsection{Fundamentos Teóricos}

En la Figura 6.1 se muestra el esquema básico del demodulador propuesto basado en una red pasiva de $N$ puertos. La arquitectura se compone de un puerto $P_{1}$ donde se aplica el oscilador local, $K$ puertos de entrada conectados a antenas, y $M$ puertos de salida conectados a detectores de potencia. El número total de puertos del sistema es $M+K+1=N$. Similar al demodulador de seis puertos, la señal de oscilador local y las señales recibidas por las antenas se combinan en los distintos puertos de salida $P_{K+2}$ a $P_{N}$. Debido a la operación no lineal que introducen los detectores de potencia se produce la conversión de frecuencia. La finalidad del diseño del demodulador de $N$ puertos es obtener

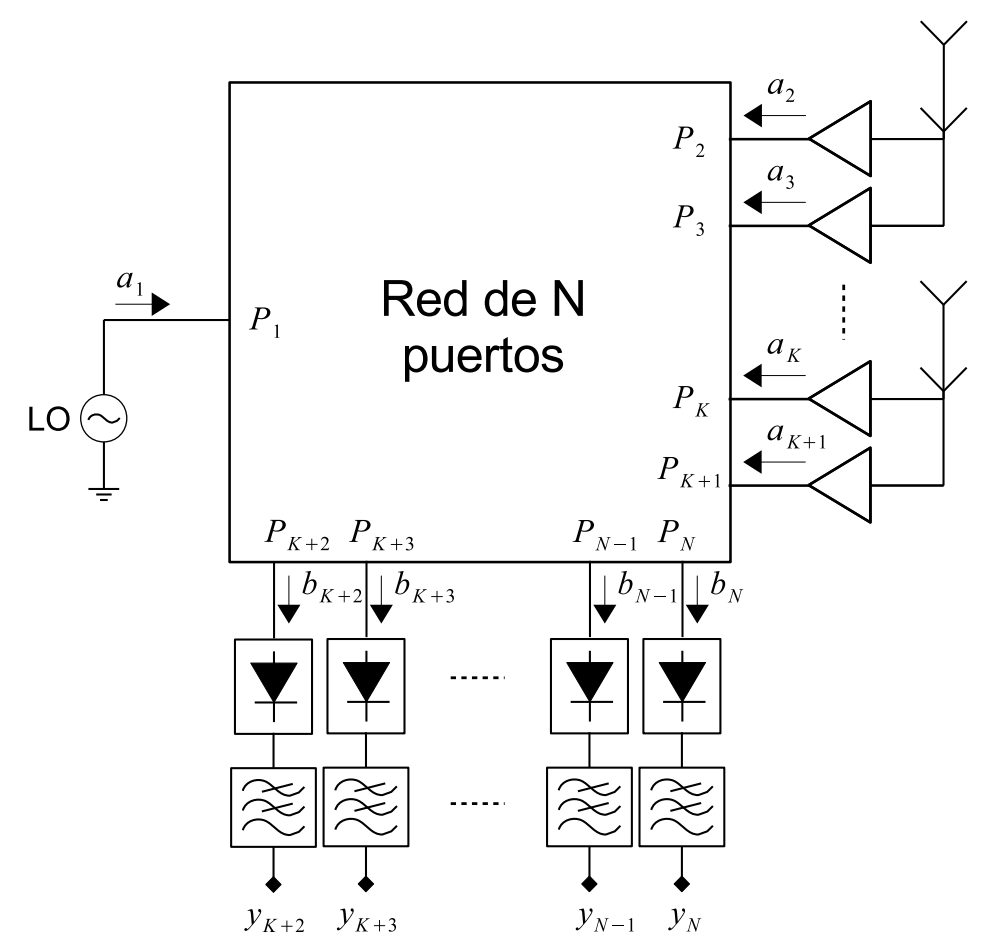

Figura 6.1: Demodulador de N puertos.

las envolventes complejas provenientes de $K$ señales recibidas a partir de $M$ mediciones de potencia $\left(y_{K+2}, \ldots, y_{N}\right)$. Una restricción importante del diseño es minimizar la cantidad de detectores de potencia para reducir la complejidad y el consumo de energía del circuito.

\subsubsection{Modelo Analítico}

Se modela el comportamiento de la red de $\mathrm{N}$ puertos utilizando su matriz de parámetros S, la cual se divide en cuatro matrices $S_{A} \in C^{(K+1) \times(K+1)}, S_{B} \in C^{(K+1) \times M}, S_{C} \in$ 
$C^{M \times(K+1)}$, y $S_{D} \in C^{M \times M}$, de la siguiente manera

$$
\left[\begin{array}{c}
b_{1} \\
\vdots \\
b_{N}
\end{array}\right]=\left[\begin{array}{cc}
S_{A} & S_{B} \\
S_{C} & S_{D}
\end{array}\right]\left[\begin{array}{c}
a_{1} \\
\vdots \\
a_{N}
\end{array}\right]
$$

donde $S_{A}$ contiene los parámetros relacionados a los puertos de entrada, $S_{D}$ a los puertos de salida, y $S_{B}$ y $S_{C}$ contienen los parámetros de transferencia entre las entradas y las salidas. Para que el sistema de la Figura 6.1 funcione adecuadamente como un demodulador, los parámetros en $S_{A}$ deben ser nulos. En otras palabras, la estructura debe asegurar buena aislación entre los puertos de entrada (antenas y oscilador local) y buena adaptación en dichos puertos. Asumiendo que los detectores están perfectamente adaptados a los puertos de la red, es decir que $a_{i}=0 \forall i \in\{K+2, \ldots, N\}$, las señales de salida en (6.1) resultan

$$
\left[\begin{array}{c}
b_{K+2} \\
\vdots \\
b_{N}
\end{array}\right]=S_{C}\left[\begin{array}{c}
a_{1} \\
\vdots \\
a_{K+1}
\end{array}\right]
$$

Notar que, $S_{D}$ no influye en la relación anterior debido a la condición ideal de adaptación de los detectores. Sin embargo, en la práctica esta característica no es perfecta por lo tanto los parámetros en $S_{D}$ deben ser cercanos a cero para reducir desbalances y acoplamientos entre las mediciones de potencia. Para simplificar la notación, la matriz en (6.1) se define como $S$ y cada elemento se denota como $s_{i j}$ donde $i$ indica la fila de la matriz y $j$ la columna. Entonces, debido a la adaptación de los detectores, la potencia promedio de cada salida $P_{i}$ con $i \in\{K+2, \ldots, N\}$ se obtiene como

$$
\begin{aligned}
\bar{p}_{i} & =\frac{\left|b_{i}\right|^{2}}{2} \\
& =\frac{1}{2}\left|\sum_{n=1}^{K+1} s_{i n} a_{n}\right|^{2} \\
& =\frac{1}{2}\left(\sum_{n=1}^{K+1} s_{i n} a_{n}\right)\left(\sum_{k=1}^{K+1} s_{i k} a_{k}\right)^{*} \\
& =\frac{1}{2} \sum_{n=1}^{K+1}\left|s_{i n} a_{n}\right|^{2}+\sum_{n=1}^{K+1} \sum_{k=n+1}^{K+1} \Re\left\{s_{i n} a_{n} s_{i k}^{*} a_{k}^{*}\right\}
\end{aligned}
$$

donde el operador $\Re\{\cdot\}$ calcula la parte real y se utiliza para simplificar la notación juntando términos en (6.3) de la siguiente manera

$$
\Re\left\{s_{i j} a_{j} s_{i k}^{*} a_{k}^{*}\right\}=\frac{1}{2}\left(s_{i j} a_{j} s_{i k}^{*} a_{k}^{*}+s_{i j}^{*} a_{j}^{*} s_{i k} a_{k}\right) .
$$

La operación típica de un demodulador consiste en multiplicar la señal recibida y la señal del oscilador local para obtener la componente de banda base deseada. En la ecuación (6.3) dicha componente está presente junto con otros productos no deseados. A continuación, se separa el término de interés y se remarca en color rojo

$$
\bar{p}_{i}=\frac{1}{2} \sum_{n=1}^{K+1}\left|s_{i n} a_{n}\right|^{2}+\sum_{n=2}^{K+1} \sum_{k=n+1}^{K+1} \Re\left\{s_{i n} a_{n} s_{i k}^{*} a_{k}^{*}\right\}+\sum_{n=2}^{K+1} \Re\left\{s_{i 1} a_{1} s_{i n}^{*} a_{n}^{*}\right\} .
$$


El objetivo del demodulador consiste en obtener las envolventes complejas de las señales recibidas $\left[a_{2}, \ldots, a_{K+1}\right]$ separando del la ecuación anterior el término en color rojo. Notar que, le expresión (6.3) contempla el caso de un demodulador de seis puertos, donde $N=6$, $K=1$ y $M=4$, en cuyo caso (6.3) se reduce a

$$
\bar{p}_{i}=\frac{1}{2}\left(\left|s_{i 1} a_{1}\right|^{2}+\left|s_{i 2} a_{2}\right|^{2}\right)+\Re\left\{s_{i 1} a_{1} s_{i 2}^{*} a_{2}^{*}\right\},
$$

la cual coincide con las expresiones (2.17)-(2.20). Hasta este punto, las señales de entrada y salida se representan por amplitudes complejas constantes. A continuación, se describen las expresiones para señales variables en el tiempo.

\section{Formulación de las Señales}

La señal del oscilador local se representa de la siguiente manera

$$
x_{O L}(t)=\left|x_{1}\right| \cos \left(2 \pi f_{r} t+\angle x_{1}\right)=\Re\left\{x_{1} e^{j 2 \pi f_{r} t}\right\}
$$

donde $x_{1}$ es la amplitud compleja del oscilador compuesta por la ganancia y la fase inicial, y $f_{r}$ su frecuencia. Además, se define la representación analítica como

$$
a_{1}(t)=x_{1} e^{j 2 \pi f_{r} t}
$$

La expresión (6.8) se puede utilizar sin ningún cambio en (6.2). Por otro lado, las señales recibidas por las antenas constan de envolventes complejas que varían en el tiempo y se representan como

$$
\begin{aligned}
x_{i}^{P B}(t) & =\Re\left\{x_{i}(t) e^{j 2 \pi f_{c} t}\right\} \\
& =x_{i}^{I}(t) \cos \left(2 \pi f_{c} t\right)-x_{i}^{Q}(t) \sin \left(2 \pi f_{c} t\right),
\end{aligned}
$$

donde $x_{i}(t)=x_{i}^{I}(t)+j x_{i}^{Q}(t)$ es la envolvente compleja, $f_{c}$ es la frecuencia de portadora y el subindice $i$ denota el puerto de entrada $P_{i}$. La representación analítica de esta señal real resulta

$$
a_{i}(t)=x_{i}(t) e^{j 2 \pi f_{r} t} .
$$

Los parámetros $S$ de la red de $N$ puertos son valores complejos que modifican la amplitud y la fase de las señales aplicada. Por ejemplo, cuando la señal del oscilador local se inyecta en el puerto $P_{1}$ esta recorre un cierto camino hasta alcanzar el puerto de salida $P_{j}$ de la red. Debido al trayecto recorrido, la señal sufre un cambio de magnitud y fase dado por el parámetro $s_{j 1}$. Por lo tanto, la señal a la entrada del detector conectado en $P_{j}$ cuando se aplica solo la señal del oscilador en $P_{1}$ se define como

$$
\Re\left\{b_{j}(t)\right\}=\Re\left\{s_{j 1} a_{1}(t)\right\}
$$

donde $a_{i}(t)$ y $b_{j}(t)$ son representaciones analíticas de las señales. Considerando que hay múltiples entradas, la señal de salida en el puerto $P_{j}$ resulta

$$
\Re\left\{b_{j}(t)\right\}=\Re\left\{\sum_{i=1}^{K+1} s_{j i} a_{i}(t)\right\}=\Re\left\{\sum_{i=1}^{K+1} s_{j i} x_{i}(t) e^{j 2 \pi f_{c} t}\right\} .
$$




\section{Conversión de Frecuencia}

La conversión de frecuencia del demodulador se realiza por mezcla aditiva de las entradas, seguida de una operación no lineal definida por lo detectores de potencia. Dichos detectores se basan en diodos Schottky operando en la zona cuadrática cuya salida se puede representar por la serie de Taylor como

$$
p(t)=K_{0}+K_{1} q(t)+K_{2} q(t)^{2}+\ldots
$$

donde $K_{0}, K_{1}, K_{2}, \ldots$ son constantes reales, y $q(t)$ es la señal de entrada del detector definida por (6.12). Entonces, la salida del detector conectado al puerto $P_{i}$ resulta

$$
p_{i}(t) \approx K_{0}+K_{1}\left(\sum_{n=1}^{K+1} \Re\left\{s_{i n} a_{n}(t)\right\}\right)+K_{2}\left(\sum_{n=1}^{K+1} \Re\left\{s_{i n} a_{n}(t)\right\}\right)^{2}
$$

donde se desprecian los términos de orden superior (mayor a tres) debido el comportamiento cuadrático del diodo. La señal (6.14) representa la potencia instantánea a la salida del detector y contiene componentes no deseadas en alta frecuencia. Por lo tanto, a la salida se conecta un filtro pasabajos cuya frecuencia de corte es $\left|f_{r}-f_{c}\right|+B / 2$ para remover las componentes no deseadas. Como resultado a la salida del filtro se obtiene la siguiente expresión, la cual se demuestra en el Apéndice D

$$
\begin{aligned}
y_{i}(t)= & K_{0}+\frac{K_{2}}{2}\left(\sum_{n=1}^{K+1}\left|s_{i n} a_{n}(t)\right|^{2}\right)+K_{2} \sum_{n=2}^{K+1} \sum_{k=n+1}^{K+1} \Re\left\{s_{i n} s_{i k}^{*} a_{n}(t) a_{k}^{*}(t)\right\} \\
& +K_{2} \sum_{n=2}^{K+1} \Re\left\{s_{i 1} s_{i n}^{*} a_{1}(t) a_{n}^{*}(t)\right\} .
\end{aligned}
$$

Utilizando las expresiones de las señales de entrada (6.8) y (6.10) en (6.15) se obtiene

$$
\begin{aligned}
y_{i}(t)= & K_{0}+\frac{K_{2}}{2}\left|s_{i 1} x_{1}\right|^{2}+\frac{K_{2}}{2}\left(\sum_{n=2}^{K+1}\left|s_{i n} x_{n}(t)\right|^{2}\right)+K_{2} \sum_{n=2}^{K+1} \sum_{k=n+1}^{K+1} \Re\left\{s_{i n} s_{i k}^{*} x_{n}(t) x_{k}^{*}(t)\right\} \\
& +K_{2} \sum_{n=2}^{K+1} \Re\left\{s_{i 1} s_{i n}^{*} x_{1} x_{n}^{*}(t) e^{j 2 \pi\left(f_{r}-f_{c}\right) t}\right\} .
\end{aligned}
$$

En la Figura 6.2 se muestra la magnitud del espectro de la señal $y_{i}(t)$ donde se distinguen tres componentes: la componente de continua dada por el término constantes $K_{0}+\frac{K_{2}}{2}\left|s_{i 1} x_{1}\right|^{2}$; las componentes en banda base que surgen del producto entre pares de señales recibidas la cual presenta un ancho de banda $2 B$ (debido a que las dos señales tienen ancho de banda $B$ ); y el termino remarcado en rojo centrado en la frecuencia $\left|f_{r}-f_{c}\right|$. Por lo tanto, si $\left|f_{r}-r_{c}\right|>3 B / 2$ no habrá solapamiento entre las componentes y se podrá remover el término deseado por filtrado. Este esquema requiere que la frecuencia del oscilador local sera diferente de la frecuencia de portadora, lo cual define un esquema del tipo heterodino. Asumiendo un filtro ideal que extrae la componente de interés sin distorsión, se obtiene

$$
w_{i}(t)=B P\left\{y_{i}(\tau)\right\}(t)=K_{2} \sum_{n=2}^{K+1} \Re\left\{s_{i 1} s_{i n}^{*} x_{1} x_{n}^{*}(t) e^{j 2 \pi\left(f_{r}-f_{c}\right) t}\right\}
$$

donde $B P\{\cdot\}$ representa la operación realizada por el filtro pasabanda. Esta operación se puede realizar en el dominio analógico, remplazando el filtro pasabajos a la salida de los 


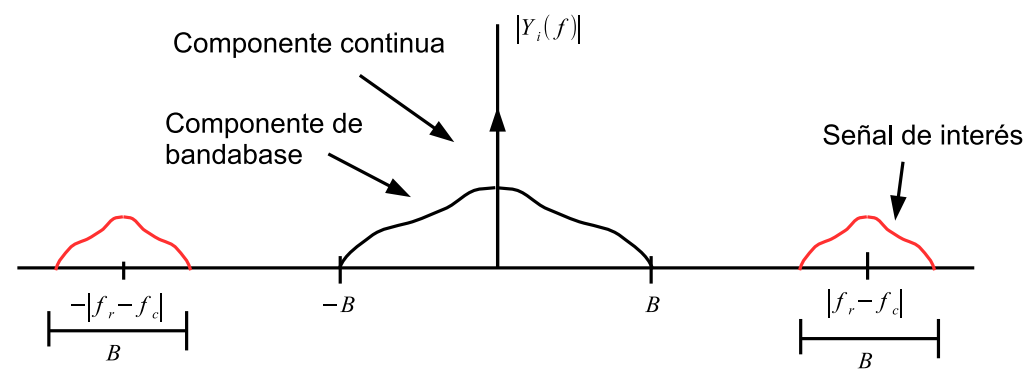

Figura 6.2: Magnitud del espectro de la señal de salida $y_{i}(t)$.

detectores por un filtro pasabanda o también en el dominio digital una vez muestreada la seãnl $y_{i}(t)$. Al considerar un esquema homodino, como el utilizado en [41] donde $f_{r}-f_{c}=$ $0 \mathrm{~Hz}$, todas las componentes en (6.16) se encuentran en banda base y no pueden ser separadas por filtrado. En su lugar, se cancelan las componentes no deseadas realizando combinaciones lineales de las mediciones de potencia.

\subsubsection{Ecuaciones de Demodulación}

A partir de (6.17) se obtienen las envolventes complejas $x_{n}(t)$ de la siguiente manera: En primer lugar, se reescribe $w_{i}(t)$ utilizando la igualdad $\Re\{x y\}=\Re\{x\} \Re\{y\}-\Im\{x\} \Im\{y\}$ $(\Im\{x\}$ calcula la parte imaginaria de $x)$, dando como resultado

$$
w_{i}(t)=K_{2} \sum_{n=2}^{K+1} \Re\left\{s_{i 1} s_{i n}^{*} x_{1}\right\} \Re\left\{x_{n}^{*}(t) e^{j 2 \pi\left(f_{r}-f_{c}\right) t}\right\}-\Im\left\{s_{i 1} s_{i n}^{*} x_{1}\right\} \Im\left\{x_{n}^{*}(t) e^{j 2 \pi\left(f_{r}-f_{c}\right) t}\right\} .
$$

Luego, se consideran las múltiples señales de salida $w_{i}(t)$ para plantear el siguiente sistema lineal

$$
\begin{array}{r}
{\left[\begin{array}{c}
w_{K+2}(t) \\
\vdots \\
w_{N}(t)
\end{array}\right]=K_{2}\left[\begin{array}{ccccc}
h_{(K+2) 2}^{R e} & -h_{(K+2) 2}^{I m} & \cdots & h_{(K+2)(K+1)}^{R e} & -h_{(K+2)(K+1)}^{I m} \\
\vdots & \vdots & \ddots & \vdots & \vdots \\
h_{N 2}^{R e} & -h_{N 2}^{I m} & \cdots & h_{N(K+1)}^{R e} & -h_{N(K+1)}^{I m}
\end{array}\right] \times} \\
\\
\quad\left[\begin{array}{c}
\Re\left\{x_{2}^{*}(t) x_{1} e^{j 2 \pi\left(f_{r}-f_{c}\right) t}\right\} \\
\Im\left\{x_{2}^{*}(t) x_{1} e^{j 2 \pi\left(f_{r}-f_{c}\right) t}\right\} \\
\vdots \\
\Re\left\{x_{K+1}^{*}(t) x_{1} e^{j 2 \pi\left(f_{r}-f_{c}\right) t}\right\} \\
\Im\left\{x_{K+1}^{*}(t) x_{1} e^{j 2 \pi\left(f_{r}-f_{c}\right) t}\right\}
\end{array}\right]
\end{array}
$$

donde

$$
\begin{aligned}
& h_{i j}^{\Re}=\Re\left\{s_{i 1} s_{i j}^{*}\right\} \\
& h_{i j}^{\Im}=\Im\left\{s_{i 1} s_{i j}^{*}\right\} .
\end{aligned}
$$

Para despejar las $K$ envolventes complejas, el rango de la matriz en (6.19) debe ser mayor o igual que $2 K$.

Para el ejemplo de demodulador de seis puertos, se utiliza la expresión (6.19) considerando una única señal recibida $a_{2}(t)=x_{2}(t) e^{j 2 \pi f_{c} t}$, la señal del oscilador local $a_{1}(t)=$ 
$x_{1} e^{j 2 \pi f_{r} t}$ y cuatro mediciones de potencia. Por lo tanto, el demodulador de seis puertos heterodino se convierte en

$$
\left[\begin{array}{c}
w_{3}(t) \\
w_{4}(t) \\
w_{5}(t) \\
w_{6}(t)
\end{array}\right]=K_{2}\left[\begin{array}{cc}
-1 / 4 & 0 \\
1 / 4 & 0 \\
0 & -1 / 4 \\
0 & 1 / 4
\end{array}\right]\left[\begin{array}{l}
\Re\left\{x_{2}^{*}(t) x_{1} e^{j 2 \pi\left(f_{r}-f_{c}\right) t}\right\} \\
\Im\left\{x_{2}^{*}(t) x_{1} e^{j 2 \pi\left(f_{r}-f_{c}\right) t}\right\}
\end{array}\right]
$$

donde los valores $s_{i j}$ de remplazaron por los parámtros ideales (2.12). Dado que el rango de la matriz anterior coincide con el número de incógnitas, se calcula la pseudoinversa (de Moore-Penrose), que se obtiene de la siguiente operación $A=\left(B^{H} B\right)^{-1} B^{H}$ donde $A$ es la pseudoinversa de $B$. A partir de la pseudoinversa se calculan las envolventes complejas como

$$
\left[\begin{array}{c}
\Re\left\{x_{2}^{*}(t) e^{j 2 \pi\left(f_{r}-f_{c}\right) t}\right\} \\
\Im\left\{x_{2}^{*}(t) e^{j 2 \pi\left(f_{r}-f_{c}\right) t}\right\}
\end{array}\right]=\frac{2}{K_{2}}\left[\begin{array}{cccc}
-1 & 1 & 0 & 0 \\
0 & 0 & -1 & 1
\end{array}\right]\left[\begin{array}{l}
w_{3}(t) \\
w_{4}(t) \\
w_{5}(t) \\
w_{6}(t)
\end{array}\right] .
$$

El resultado (6.23) coincide con la solución presentada en (2.34) para el demodulador de seis puertos tradicional (homodino). Sin embargo, notar que utilizando el esquema heterodino la expresión (6.22) se puede resolver para las envolventes complejas utilizando solo dos mediciones, dado que las componentes no deseadas se eliminan por filtrado en una instancia previa,

$$
\left[\begin{array}{c}
\Re\left\{x_{2}^{*}(t) e^{j 2 \pi\left(f_{r}-f_{c}\right) t}\right\} \\
\Im\left\{x_{2}^{*}(t) e^{j 2 \pi\left(f_{r}-f_{c}\right) t}\right\}
\end{array}\right]=\frac{4}{K_{2}}\left[\begin{array}{c}
w_{4}(t) \\
w_{6}(t)
\end{array}\right] .
$$

Debido a la redundancia en las mediciones de potencia se plantea un ejemplo basado en la estructura de siete puertos utilizada para implementar el demodulador de seis puertos mostrado en la Figura 2.7. A continuación se presenta un ejemplo basado en una estructura de siete puertos.

\subsubsection{Demodulador de Siete Puerto I}

La Figura 6.3 presenta un demodulador de siete puertos, el cual se conecta a dos antenas receptoras $(K=2)$, un oscilador local y cuatro detectores de potencia $(M=4)$. Las señales de entrada son $a_{2}(t)$ y $a_{3}(t)$ y el oscilador local $a_{1}(t)$. La relación entre los puertos de salida y los puertos de entrada de la estructura de siete puertos está definida por los parámetros $S$ presentados en (3.1). Notar que se utiliza la misma numeración de puertos. Entonces, las señales de salida en los puertos $P_{4}-P_{7}$ resultan

$$
\left[\begin{array}{l}
b_{4}(t) \\
b_{5}(t) \\
b_{6}(t) \\
b_{7}(t)
\end{array}\right]=\frac{1}{2}\left[\begin{array}{ccc}
-1 & j & 1 \\
j & -1 & j \\
-1 & 1 & j \\
j & j & -1
\end{array}\right]\left[\begin{array}{l}
a_{1}(t) \\
a_{2}(t) \\
a_{3}(t)
\end{array}\right]
$$

y las señales de entrada se definen como

$$
\left[\begin{array}{l}
a_{1}(t) \\
a_{2}(t) \\
a_{3}(t)
\end{array}\right]=\left[\begin{array}{c}
x_{1} e^{j 2 \pi f_{r} t} \\
x_{2}(t) e^{j 2 \pi f_{c} t} \\
x_{3}(t) e^{j 2 \pi f_{c} t}
\end{array}\right]
$$




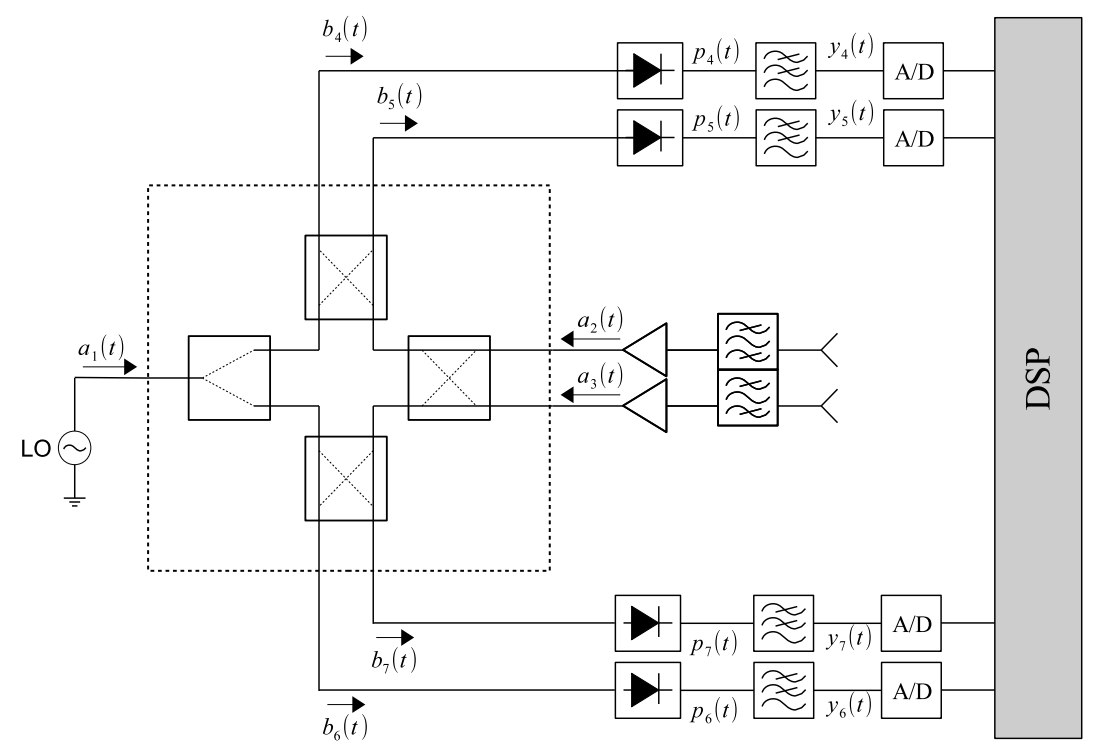

Figura 6.3: Demodulador basado en una red de siete puertos.

donde $x_{1}$ es la amplitud compleja de la señal de oscilador local y $f_{r}$ su frecuencia de portadora. $x_{2}(t)$ y $x_{3}(t)$ son las envolventes complejas de las señales recibidas por las antenas conectadas en los puertos $P_{2}$ y $P_{3}$. Cada detector mide la potencia instantánea y el filtro pasabajos se encarga de extraer las componentes de baja frecuencia resultando la señal $y_{i}(t)$

$$
\begin{aligned}
y_{i}(t)= & L P\left(p_{i}(t)\right) \\
= & K_{0}+K_{2} \frac{\left|s_{i 1} x_{1}\right|^{2}}{2}+K_{2} \frac{\left|s_{i 2} x_{2}(t)\right|^{2}}{2}+K_{2} \frac{\left|s_{i 3} x_{3}(t)\right|^{2}}{2} \\
& +K_{2} \Re\left\{s_{i 2} s_{i 3}^{*} x_{2}(t) x_{3}^{*}(t)\right\}+K_{2} \Re\left\{s_{i 1} s_{i 2}^{*} x_{1} x_{2}^{*}(t) e^{j 2 \pi\left(f_{r}-f_{c}\right) t}\right\} \\
& +\Re\left\{s_{i 1} s_{i 3}^{*} x_{1} x_{3}^{*}(t) e^{j 2 \pi\left(f_{r}-f_{c}\right) t}\right\}
\end{aligned}
$$

y al aplicar el filtro pasabanda se obtiene

$$
w_{i}(t)=K_{2} \Re\left\{s_{i 1} s_{i 2}^{*} x_{1} x_{2}^{*}(t) e^{j 2 \pi\left(f_{r}-f_{c}\right) t}\right\}+K_{2} \Re\left\{s_{i 1} s_{i 3}^{*} x_{1} x_{3}^{*}(t) e^{j 2 \pi\left(f_{r}-f_{c}\right) t}\right\}
$$

Con los datos del ejemplo y utilizando la ecuación (6.19) se obtiene

$$
\left[\begin{array}{c}
\omega_{4}(t) \\
\omega_{5}(t) \\
\omega_{6}(t) \\
\omega_{7}(t)
\end{array}\right]=\left[\begin{array}{cccc}
0 & -1 / 4 & -1 / 4 & 0 \\
0 & 1 / 4 & 1 / 4 & 0 \\
-1 / 4 & 0 & 0 & -1 / 4 \\
1 / 4 & 0 & 0 & 1 / 4
\end{array}\right]\left[\begin{array}{c}
\Re\left\{x_{1} x_{2}^{*}(t) e^{j 2 \pi\left(f_{r}-f_{c}\right) t}\right\} \\
\Im\left\{x_{1} x_{2}^{*}(t) e^{j 2 \pi\left(f_{r}-f_{c}\right) t}\right\} \\
\Re\left\{x_{1} x_{3}^{*}(t) e^{j 2 \pi\left(f_{r}-f_{c}\right) t}\right\} \\
\Im\left\{x_{1} x_{3}^{*}(t) e^{j 2 \pi\left(f_{r}-f_{c}\right) t}\right\}
\end{array}\right]
$$

El rango de la matriz anterior es (dos) menor que el número de incógnitas $(2 K)$, por lo tanto, el sistema no se puede resolver. Existirá una estructura de siete puertos que genere una matriz en (6.19) de rango cuatro? A continuación se plantea un esquema de demodulación basado en la transformada de Hilbert que permite resolver (6.29) sin cambiar el diseño de la red. 


\subsubsection{Transformada de Hilbert}

La transformada de Hilbert $(\mathrm{TH})$ permite obtener la representación analítica $\left(z_{i}(t)\right)$ de la señal basabanda real $w_{i}(t)$ de la siguiente manera

$$
z_{i}(t)=w_{i}(t)+j T H\left\{w_{i}(\tau)\right\}(t)=\sum_{n=2}^{K+1} s_{i 1} s_{i n}^{*} x_{1} x_{n}^{*}(t) e^{j 2 \pi\left(f_{r}-f_{c}\right) t} .
$$

La transformación $T H\{\cdot\}$ es una operación lineal definida por la convolución con la función $\frac{1}{\pi t}$. Entonces, remplazando los parámetros ideales en (6.33) se obtiene

$$
\left[\begin{array}{c}
z_{4}(t) \\
z_{5}(t) \\
z_{6}(t) \\
z_{7}(t)
\end{array}\right]=x_{1}\left[\begin{array}{cc}
j / 4 & -1 / 4 \\
-j / 4 & 1 / 4 \\
-1 / 4 & j / 4 \\
1 / 4 & -j / 4
\end{array}\right]\left[\begin{array}{l}
x_{2}^{*}(t) \\
x_{3}^{*}(t)
\end{array}\right] e^{j 2 \pi\left(f_{r}-f_{c}\right) t} .
$$

El sistema anterior se puede resolver dado que se tiene dos señales incógnitas y la matriz tiene rango dos. Por lo tanto calculando la pseudoinversa se obtienen las envolventes complejas como

$$
\left[\begin{array}{l}
x_{2}(t) \\
x_{3}(t)
\end{array}\right]=\left(\frac{1}{x_{1}}\left[\begin{array}{llll}
-j & j & -1 & 1 \\
-1 & 1 & -j & j
\end{array}\right]\left[\begin{array}{l}
z_{4}(t) \\
z_{5}(t) \\
z_{6}(t) \\
z_{7}(t)
\end{array}\right]\right)^{*} e^{j 2 \pi\left(f_{r}-f_{c}\right) t}
$$

Finalmente se logra demodular dos envolventes complejas a partir de cuatro mediciones de potencia. Este esquema utiliza la mitad de detectores que el demodulador de seis puertos, sin embargo obliga a reformular los algoritmos de procesamiento de señales. La complejidad de la implementación de la transformada discreta de Hilbert dependerá principalmente del ancho de banda de la señal pasabanda.

A continuación, se presentan las expresiones para un caso general de un demodulador de $N$ puertos. Al aplicar la transformación de Hilbert en (6.17) la representación analítica de $w_{i}(t)$ resulta

$$
z_{i}(t)=w_{i}(t)+j T H\left\{w_{i}(\tau)\right\}(t)=\sum_{n=2}^{K+1} s_{i 1} s_{i n}^{*} x_{1} x_{n}^{*}(t) e^{j 2 \pi\left(f_{r}-f_{c}\right) t} .
$$

A partir de $z_{i}(t)$ se plantea el siguiente sistema lineal

$$
\left[\begin{array}{c}
z_{K+2}(t) \\
\vdots \\
z_{N}(t)
\end{array}\right]=x_{1} A\left[\begin{array}{c}
x_{2}^{*}(t) \\
\vdots \\
x_{K+1}^{*}(t)
\end{array}\right] e^{j 2 \pi\left(f_{r}-f_{c}\right) t}
$$

donde la matriz $A$ se define como

$$
A=\left[\begin{array}{ccc}
s_{(K+2) 1} s_{(K+2) 2}^{*} & \cdots & s_{(K+2) 1} s_{(K+2)(K+1)}^{*} \\
\vdots & \ddots & \vdots \\
s_{N 1} s_{N 2}^{*} & \cdots & s_{N 1} s_{N(K+1)}^{*}
\end{array}\right]
$$


Observar que el sistema (6.34) se puede resolver para las envolventes $x_{i}(t)$ si el rango de la matriz $H$ es igual o mayor que $K$. En cuyo caso se obtiene la pseudoinversa $A$ y se demodulan las envolventes como

$$
\left[\begin{array}{c}
x_{2}(t) \\
\vdots \\
x_{K+1}(t)
\end{array}\right]=\left(\frac{1}{x_{1}}\left(A^{H} A\right)^{-1} A^{H}\left[\begin{array}{c}
z_{K+2}(t) \\
\vdots \\
z_{N}(t)
\end{array}\right]\right)^{*} e^{j 2 \pi\left(f_{r}-f_{c}\right) t}
$$

donde $A^{H}$ es la matriz traspuesta y conjugada (hermítica) de $A$.

\subsubsection{Validación Experimental}

Para validar las expresiones anteriores se desarrolló un prototipo modular del demodulador propuesto.

\section{Prototipo}

El prototipo consiste en la red de siete puertos, cuatro detectores de potencia y los filtros paso bajo. La red de siete puertos fue diseñada y fabricada para una frecuencia central de 1575,42 MHz en tecnología de microtira. La red se desarrolló en una placa de FR-4 de $13 \times 15 \mathrm{~cm}$ cuya permitividad relativa es $\varepsilon=4,6$. Los detectores de potencia se basan en el circuito integrado LT5538 el cual se aplica en placas separadas del mismo material. Cada detector contiene el filtro de paso bajo compuesto por un circuito RC. La Figura 6.4 muestra una fotografía de la red de siete puertos y un detector de potencia. Los detectores están conectados a los puertos correspondientes $\mathrm{P}_{4}-P_{7}$ de la estructura a través de los conectores SMA.
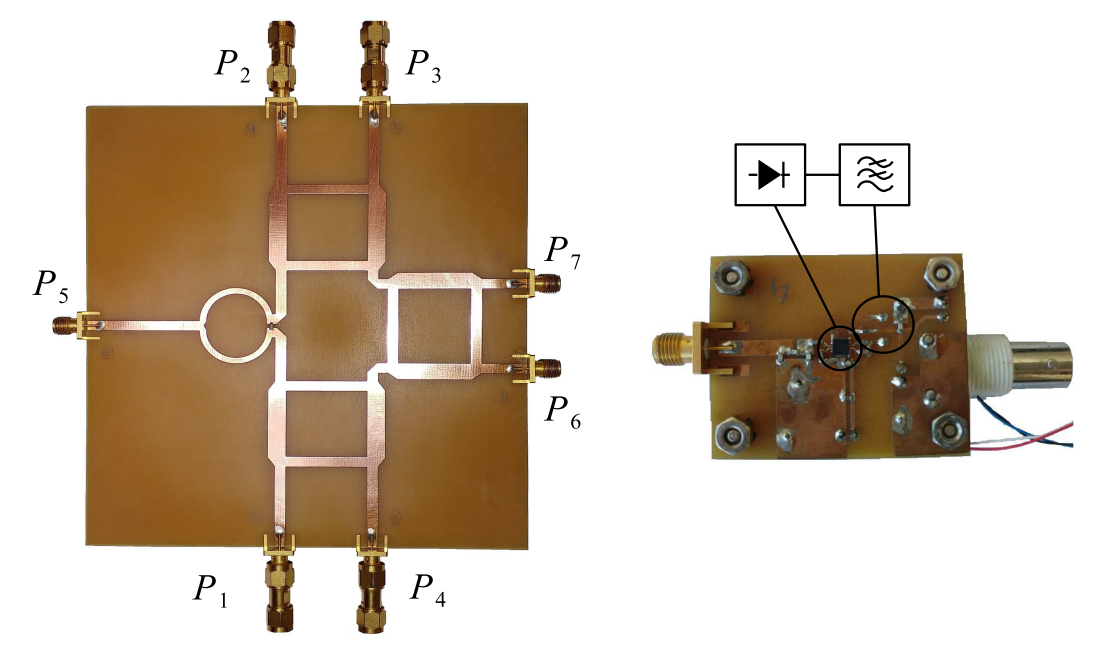

Figura 6.4: Fotografía del prototipo implementado para operar a 1575,42 MHz: red de siete puertos (izquierda) y detector de potencia (derecha). 


\section{Configuración de la Medición}

Para generar los datos de salida del sistema de siete puertos y validar el modelo teórico, se utilizan dos generadores de señales conectados a la misma referencia de $10 \mathrm{MHz}$ de la siguiente manera: el $4684 C$ de Keysight Technologies genera la señal oscilador local en el puerto P1 con una frecuencia de 1575, 4 MHz; y el N4422B de Keysight Technologies genera la señal modulada (simulando la señal recibida por las antenas) con una frecuencia portadora de 1575,42 MHz. La diferencia entre las frecuencias de portadora es de $20 \mathrm{KHz}$, de esta manera se separan las componentes deseadas en $f_{r}-f_{c}$ de las componentes en banda base como muestra la Figura 6.2. La señal modulada se aplica a la entrada de

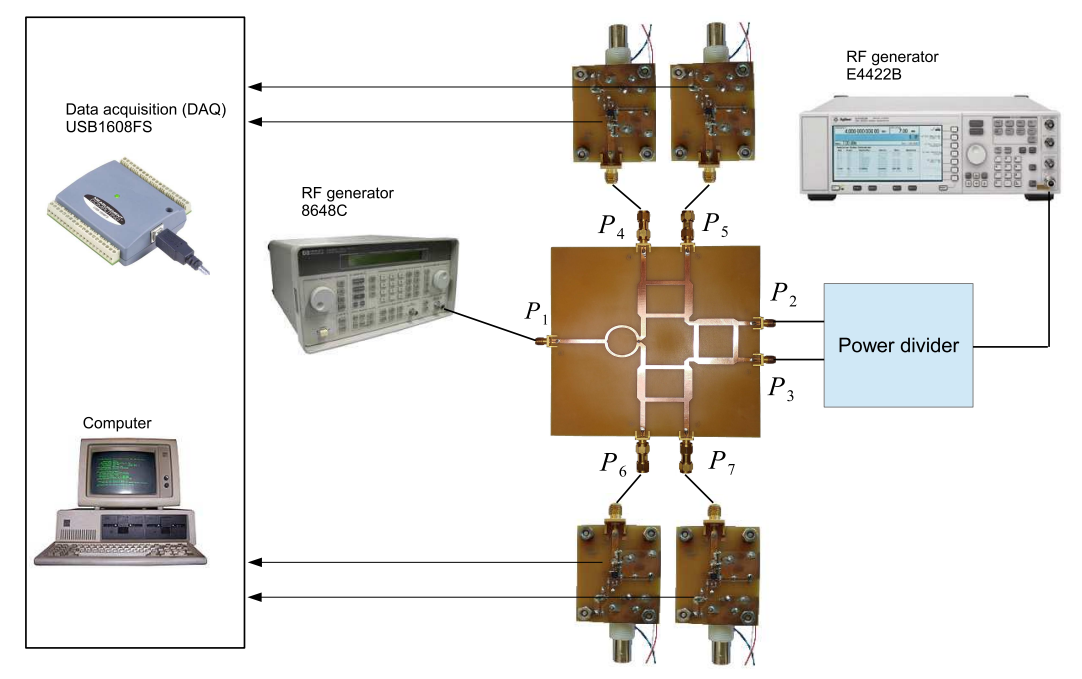

Figura 6.5: Diagrama del sistema de medición.

un divisor de potencia que proporciona dos señales de salida y las inyecta en los puertos $P_{2}$ y $P_{3}$ como muestra la Figura 6.5. Dicho dispositivo se selecciona entre un divisor de potencia Wilkinson y un acoplador híbrido de cuadratura para proporcionar distintas combinaciones de salida:

- Caso (a), se inyecta en el puerto $P_{2}$ y $P_{3}$ la misma señal modulada usando un divisor de potencia wilkinson, es decir que $x_{2}(t)=x_{3}(t)$.

- Caso (b), se utiliza el acoplador híbrido en cuadratura para generar dos señales modulada de la misma amplitud pero en cuadratura, $x_{2}(t)=x_{3}(t) e^{j \pi / 2}$.

- Caso (c), igual que el caso (b) pero con signo contrario (se inyecta la señal del generador en la otra entrada del acoplador), entonces $x_{2}(t)=x_{3}(t) e^{-j \pi / 2}$.

- Caso (d), se inyecta la señal modulada en el puerto P2, es decir que $x_{3}(t)=0$.

Para todos los casos la modulación es un tono de $f_{m}=1 \mathrm{KHz}$ modulado en fase, es decir, la señal de banda base $x(t)$ es

$$
x(t)=G_{x} e^{j \beta \sin \left(2 \pi f_{m} t\right)}
$$

donde $G_{x}$ es la magnitud constante de la envolvente y $\beta=1$ es la desviación máxima de la fase. La potencia de los generadores se configura en $P_{x}=-30 \mathrm{dBm}$ para la señal inyectada 
a la entrada del divisor de potencia y $P_{1}=-20 \mathrm{dBm}$ para el oscilador local. El ancho de banda de la señal modulada está dado por $B \approx 2(1+\beta) f_{m}=4 \mathrm{KHz}$. Finalmente, se toman muestras de las cuatro señales de salida $y_{4}(t)-y_{7}(t)$ utilizando un convertidor analógicodigital USB1608FS de Measuring Computing, el cual permite muestrear cuatro canales con una frecuencia de 90 Ksamples/s por cada canal. Para cada caso (a)-(d), se guardan las muestras de $y_{i}[n]=y_{i}\left(n T_{s}\right)(i \in\{4,5,6,7\})$ en MATLAB para su procesamiento.

\section{Resultados}

Para obtener las envolventes complejas a partir de los valores de las secuencias $y_{i}[n]$, primero se debe aplicar el filtro pasabanda para eliminar las componentes no deseadas. Por lo tanto, se implementa un filtro FIR para obtener las secuencias $w_{i}[n]$. Luego las expresiones analíticas $z_{i}[n]$ se obtienen aplicando la transformada de Hilbert discreta implementada en MATLAB. Finalmente, las envolventes complejas $x_{2}[n]$ y $x_{3}[n]$ se obtienen calculando (6.32). La Figura 6.6 presenta magnitud y la fase de las envolventes complejas para cada caso (a)-(d). Para comparar los valores obtenidos a partir de las mediciones con

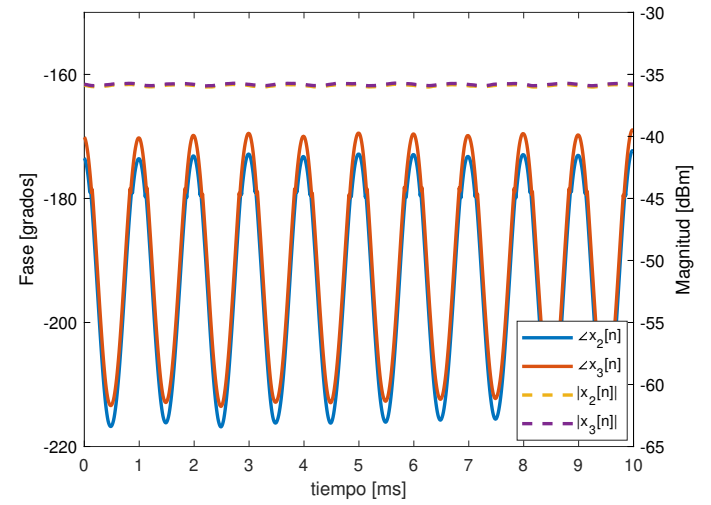

(a)

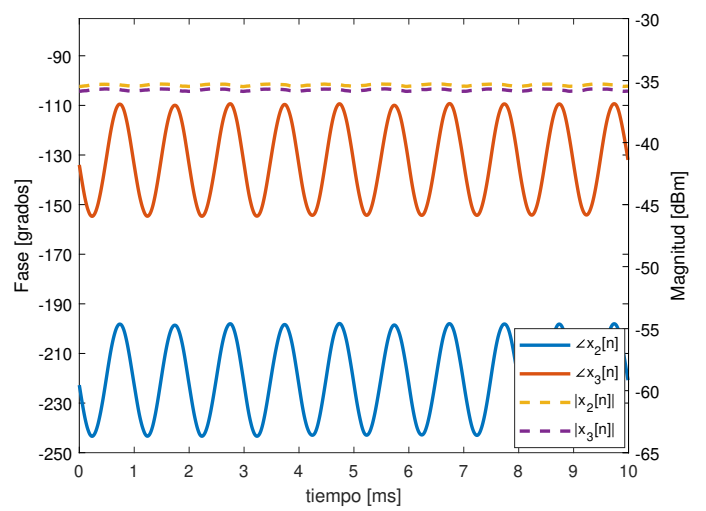

(c)

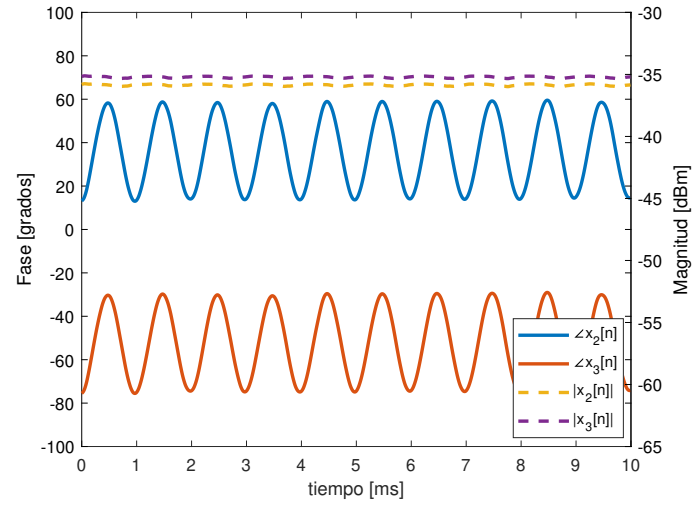

(b)

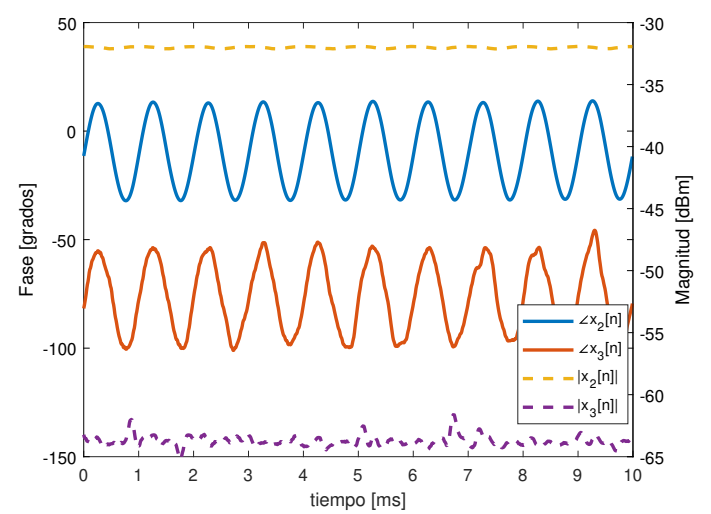

(d)

Figura 6.6: Resultados medidos. Magnitud y fase de las envolventes complejas $x_{2}[n]$ y $x_{3}[n]$ para los casos (a)-(d).

los valores esperados, se calcula la magnitud promedio y la diferencia de fase promedio. Los valores se presenta en el Cuadro 6.1. Se observa una buena correspondencia entre los valores esperados y los valores obtenidos del prototipo. Las diferencias en los valores de magnitud se deben principalmente por las pérdidas de inserción de las conexiones 


\begin{tabular}{lll}
\hline \hline Caso & Valor medido & Valor esperado \\
\hline (a) & $P_{2}=-35,6 \mathrm{dBm}$ & $P_{2}=-33 \mathrm{dBm}$ \\
& $P_{3}=-35 \mathrm{dBm}$ & $P_{3}=-33 \mathrm{dBm}$ \\
& $\Delta \phi=3,33^{\circ}$ & $\Delta \phi=0^{\circ}$ \\
(b) & $P_{2}=-35,4 \mathrm{dBm}$ & $P_{2}=-33 \mathrm{dBm}$ \\
& $P_{3}=-35,3 \mathrm{dBm}$ & $P_{3}=-33 \mathrm{dBm}$ \\
& $\Delta \phi=-88,56^{\circ}$ & $\Delta \phi=-90^{\circ}$ \\
(c) & $P_{2}=-35,8 \mathrm{dBm}$ & $P_{2}=-33 \mathrm{dBm}$ \\
& $P_{3}=-35,1 \mathrm{dBm}$ & $P_{2}=-33 \mathrm{dBm}$ \\
& $\Delta \phi=88,67^{\circ}$ & $\Delta \phi=90^{\circ}$ \\
(d) & $P_{2}=-32,2 \mathrm{dBm}$ & $P_{2}=-30 \mathrm{dBm}$ \\
& $P_{3}=-64 \mathrm{dBm}$ & $P_{3}=-\infty \mathrm{dBm}$ \\
\hline
\end{tabular}

Cuadro 6.1: Tabla con los valores de potencia de entrada en los puertos $P_{2}$ y $P_{3}$.

utilizadas y las pérdidas no ideales del divisor Wilkinson y el acoplador híbrido.

\subsection{Demodulador de Siete Puertos II}

En la Sección 6.2.4 se presentó un esquema de demodulación de $N$ puertos basado en la transformada de Hilbert. Notar que, la expresión (6.34) establece que la cantidad mínima de detectores de potencia necesarios para demodular $K$ envolventes complejas es $K$. A continuación se plantea un diseño de siete puertos el cual se conecta a tres antenas, tres detectores de potencia y un oscilador local. El demodulador de siete puertos propuesto en este ejemplo se muestra en la Figura 6.7. La estructura de siete puertos utilizada es la misma que en el esquema I, sin embargo, la configuración de sus puertos y la conexión con las antenas y los detectores es diferente. El oscilador local se conecta en el puerto $P_{1}$, y tres antenas en los puertos $P_{2}, P_{3}$ y $P_{4}$. Las señales de entrada son $a_{2}(t), a_{3}(t), a_{4}(t)$ y el oscilador local $a_{1}(t)$. Con esta configuración de puertos, la estructura de siete puertos genera tres combinaciones lineales de las entradas en los puertos de salida $P_{5}-P_{7}$, donde están conectados los detectores de potencia.

\subsubsection{Modelo Teórico}

La matriz de parámetros $S$ de la red de siete puertos se presenta en (3.1). Se debe tener en cuenta que la configuración de puertos mostrada en la Figura 6.7 es diferente de la numeración utilizada en (3.1). Por lo tanto, seleccionando adecuadamente parámetros, se obtiene la siguiente relación entre las señales emergentes e incidentes

$$
\left[\begin{array}{l}
b_{5}(t) \\
b_{6}(t) \\
b_{7}(t)
\end{array}\right]=\frac{1}{2}\left[\begin{array}{cccc}
-1 & -1 & j & j \\
j & 1 & j & -1 \\
1 & j & -1 & j
\end{array}\right]\left[\begin{array}{l}
a_{1}(t) \\
a_{2}(t) \\
a_{3}(t) \\
a_{4}(t)
\end{array}\right] .
$$




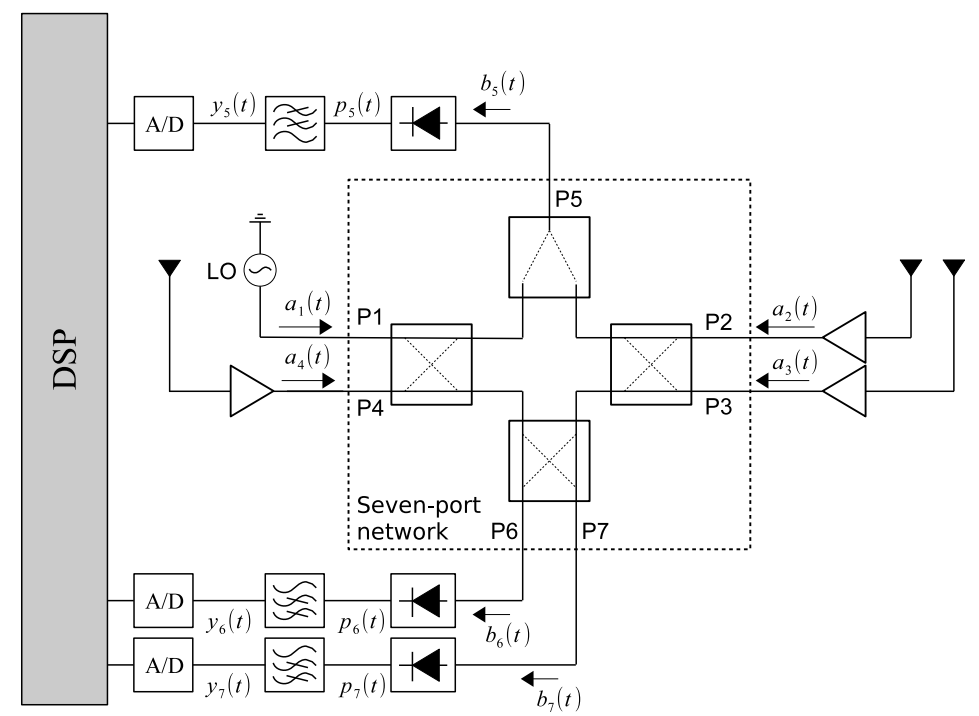

Figura 6.7: Demodulador basado en una red de siete puertos.

Las señales de entrada se definen a través del siguiente vector

$$
\left[\begin{array}{c}
a_{1}(t) \\
a_{2}(t) \\
a_{3}(t) \\
a_{4}(t)
\end{array}\right]=\left[\begin{array}{c}
x_{1} e^{j 2 \pi f_{r} t} \\
x_{2}(t) e^{j 2 \pi f_{c} t} \\
x_{3}(t) e^{j 2 \pi f_{c} t} \\
x_{4}(t) e^{j 2 \pi f_{c} t}
\end{array}\right]
$$

donde $x_{1}$ es la amplitud compleja del OL y $f_{r}$ su frecuencia. Asimismo, $x_{2}(t), x_{3}(t)$ y $x_{4}(t)$ son las envolventes complejas de las señales recibidas por las distintas antenas. Siguiendo la formulación desarrollada en la Sección 6.2 se obtienen las señales $w_{i}(t)$ con $i \in\{5,6,7\}$ aplicando el correspondiente filtrado pasabanda

$$
\omega_{i}(t)=\Re\left\{a_{1} e^{j 2 \pi\left(f_{r}-f_{c}\right) t} s_{i 1}\left(s_{i 2}^{*} x_{2}^{*}(t)+s_{i 3}^{*} x_{3}^{*}(t)+s_{i 4}^{*} x_{4}^{*}(t)\right)\right\} .
$$

Luego se calcula la señal analítica de $w_{i}(t)$ a partir de la transformada de Hilbert obteniendo

$$
z_{i}(t)=x_{1} e^{j 2 \pi\left(f_{r}-f_{c}\right) t} s_{i 1}\left(s_{i 2}^{*} x_{2}^{*}(t)+s_{i 3}^{*} x_{3}^{*}(t)+s_{i 4}^{*} x_{4}^{*}(t)\right) .
$$

Considerando las tres señales de salida, la expresión anterior se escribe en forma matricial como

$$
\left[\begin{array}{l}
z_{5}(t) \\
z_{6}(t) \\
z_{7}(t)
\end{array}\right]=x_{1} A\left[\begin{array}{c}
x_{2}^{*}(t) \\
x_{3}^{*}(t) \\
x_{4}^{*}(t)
\end{array}\right] e^{j 2 \pi\left(f_{r}-f_{c}\right) t}
$$

donde

$$
A=\frac{1}{4}\left[\begin{array}{ccc}
1 & j & j \\
j & 1 & -j \\
-j & -1 & -j
\end{array}\right]
$$

Luego, se calcula la matriz inversa $A^{-1}$ y se obtienen las envolventes complejas

$$
\left[\begin{array}{l}
x_{2}(t) \\
x_{3}(t) \\
x_{4}(t)
\end{array}\right]=\left(\frac{1}{x_{1}}\left[\begin{array}{ccc}
2 & 1-j & 1+j \\
-2 j & 1-j & -1-j \\
0 & 2 j & 2 j
\end{array}\right]\left[\begin{array}{c}
z_{5}(t) \\
z_{6}(t) \\
z_{7}(t)
\end{array}\right]\right)^{*} e^{j 2 \pi\left(f_{r}-f_{c}\right) t}
$$




\subsubsection{Validación Experimental}

Para la validación se desarrolla el prototipo utilizando la misma estructura de siete puerto implementada anteriormente y tres detectores de potencia conectados como muestra la Figura 6.8.

\section{Configuración de la Medición}

La configuración de sistema es similar al caso del demodulador esquema I. Sin embargo, se requiere un generador adicional para inyectar las respectivas señales de entrada. Se utiliza $4684 C$ para el OL en el puerto P1 con una frecuencia de $1575,4 \mathrm{MHz}$; el de $N 5172 B$ de Keysight Technologies genera la señal modulada en el puerto $P_{4}$ con una frecuencia de portadora de 1575, $42 \mathrm{MHz}$; y el $N 4422 B$ de Keysight Technologies genera las señales moduladas en los puertos $P_{2}$ y $P_{3}$ con una frecuencia de portadora de $1575,42 \mathrm{MHz}$. La modulación generada por $N 4422 B$ es un tono de $f_{m 1}=400 \mathrm{~Hz}$ modulado en fase (6.37) y se aplica a la entrada de el divisor. Mientras que la señal modulada por $N 4422 B$ presenta la misma modulación pero con una frecuencia $f_{m 2}=1 \mathrm{KHz}$. Siguiendo esta configuración miden dos situaciones: caso (a) poniendo el acoplador híbrido en cuadratura como dispositivo divisor; y caso (b) con divisor Wilkinson. La potencia del oscilador se con-

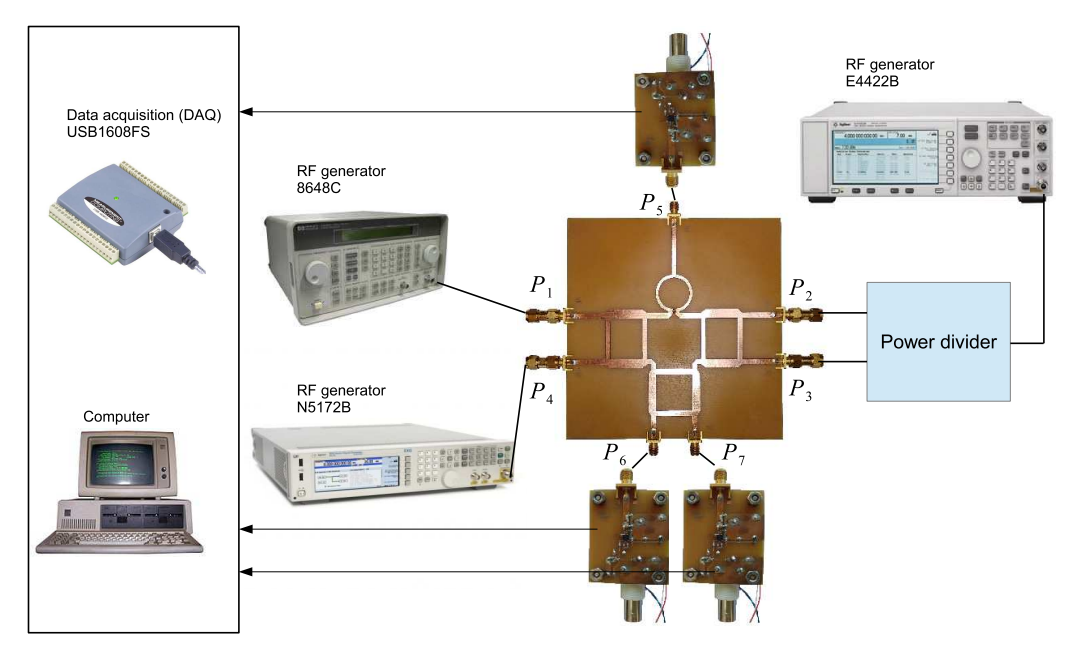

Figura 6.8: Diagrama del sistema de medición.

figura en $P_{1}=-20 \mathrm{dBm}$ y las de los generadores que producen las señales moduladas en $P_{x}=-30 \mathrm{dBm}$. Las muestras se obtienen a partir del USB1608Fs y se guardan en MATLAB

\section{Resultados}

Al igual que en el ejemplo del esquema I, se implementa el filtro pasabanda y la transformación de Hilbert en MATLAB. Luego de aplicar ambas operaciones se obtienen las señales analíticas $z_{i}[n]$. El problema inverso (6.44) resulta

$$
\left[\begin{array}{c}
x_{2}[n] \\
x_{3}[n] \\
x_{4}[n]
\end{array}\right]=\frac{1}{\left|x_{1}^{*}\right|}\left(H^{-1}\right)^{*}\left[\begin{array}{c}
z_{5}[n] \\
z_{6}[n] \\
z_{7}[n]
\end{array}\right]^{*} e^{j 2 \pi \frac{\left(f_{r}-f_{c}\right)}{f_{s}} n} .
$$


donde $\left|x_{1}\right|=10^{P_{1} / 20}$ y la fase se observa en los resultados de las envolventes complejas como un valor de fase constante aplicada a las tres señales. La Figura 6.9(a) presenta la

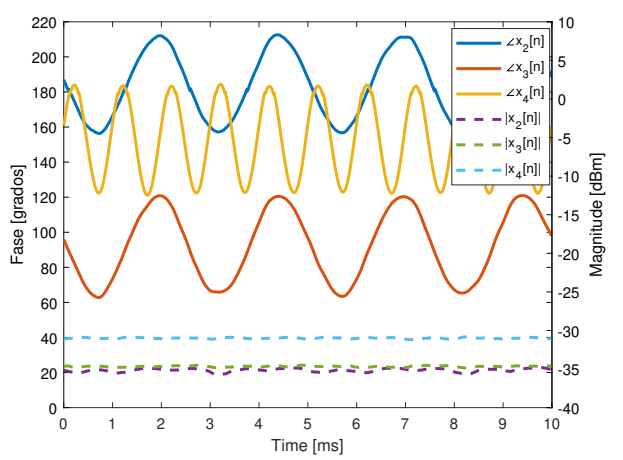

(a)

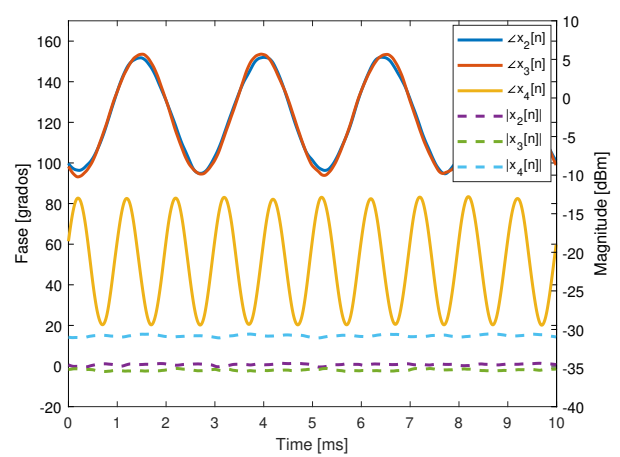

(b)

Figura 6.9: Resultados medidos. Magnitud y fase de las envolventes complejas $x_{2}[n], x_{3}[n]$ y $x_{4}[n]$ para el caso (a) y (b).

magnitud y fase de las envolventes complejas obtenidas el colocar el acoplador híbrido en cuadratura en los puertos $P_{2}$ y $P_{3}$ y la Figura 6.9(b) al colocar el divisor Wilkinson. Para comparar los resultados de las mediciones y los resultados esperados de la teoría se calcula la magnitud y la diferencia de fase entre $x_{2}[n]$ y $x_{3}[n]$ promedio. En el Cuadro 6.3.2 se presentan los valores obtenidos.

\begin{tabular}{lll}
\hline \hline Caso & Valor medido & Valor esperado \\
\hline (a) & $P_{2}=-35,08 \mathrm{dBm}$ & $P_{2}=-33 \mathrm{dBm}$ \\
& $P_{3}=-34,6 \mathrm{dBm}$ & $P_{3}=-33 \mathrm{dBm}$ \\
& $P_{4}=-30,9 \mathrm{dBm}$ & $P_{4}=-30 \mathrm{dBm}$ \\
& $\Delta \phi=-91,2^{\circ}$ & $\Delta \phi=-90^{\circ}$ \\
(b) & $P_{2}=-34,5 \mathrm{dBm}$ & $P_{2}=-33 \mathrm{dBm}$ \\
& $P_{3}=-35,2 \mathrm{dBm}$ & $P_{3}=-33 \mathrm{dBm}$ \\
& $P_{3}=-30,8 \mathrm{dBm}$ & $P_{4}=-30 \mathrm{dBm}$ \\
& $\Delta \phi=-0,18^{\circ}$ & $\Delta \phi=0^{\circ}$ \\
\hline
\end{tabular}

Cuadro 6.2: Tabla con los valores de potencia de entrada en los puertos $P_{2}$ y $P_{3}$.

Notar que la potencia de las señales en los puertos $P_{2}$ y $P_{3}$ es $3 \mathrm{~dB}$ inferior a la potencia aplicada por el generador $P_{x}$ debido a la presencia de divisor Wilkinson o el acoplador híbrido. Los resultados anteriores presentan las tres envolventes complejas de magnitud constante y fase sinusoidal obtenidas a partir de tres mediciones de potencia. Se observa una buena correspondencia entre los valores esperados por el modelo teórico y los valores obtenidos por las mediciones sobre el prototipo.

\subsection{Conclusión}

En este capítulo se presentaron los fundamentos teóricos para el diseño de un demodulador de $N$ puertos capaz de recuperar las envolventes complejas de múltiples señales recibidas por un arreglo de sensores. El diseño propuesto logra reducir la complejidad 
del circuito analógico minimizando la cantidad de detectores de potencia utilizados, y aumenta la complejidad del circuito digital incorporando etapas de filtrado adicionales. Utilizando los conceptos desarrollados, se diseñaron, implementaron y validaron dos arquitecturas basadas en una red de siete puertos. Uno de los dispositivos es un demodulador capaz de recuperar las envolventes complejas de las señales recibidas por dos antenas a partir de cuatro mediciones de potencia, y el otro dispositivo permite demodular tres envolventes complejas provenientes de tres antenas diferentes a partir de tres mediciones de potencia. Los datos recuperados de los prototipos mostraron una buena concordancia con los resultados esperados del modelo teórico. Ambos prototipo fueron fabricados para una frecuencia de operación de 1575,42 MHz. Debido al número de detectores de potencia y antenas, ambas arquitecturas proponen una alternativa de menor costo y consumo de energía respecto a implementaciones con arquitecturas de seis puertos.

Hay varios aspectos de los demoduladores que serán estudiados en el futuro. Por ejemplo, una técnica de calibración para obtener los parámetros $H$ para reducir el error del modelo. Además, se deben estudiar algoritmos de estimación para recuperar la información de la señal cuando esta está contaminada con ruido, y realizar una validación experimental del las arquitecturas utilizando antenas. 


\section{Capítulo 7}

\section{Conclusiones}

En esta tesis, se utilizaron estructuras de siete puertos para la implementación de sistemas de modulación y demodulación para aplicaciones en arreglos de sensores. En primer lugar, se presentó un modulador de microondas diseñado para aplicaciones inalámbricas con diversidad polarimétrica. La arquitectura propuesta puede modular dos salidas simultáneamente a través de cuatro señales de banda base las cuales controlan impedancias variables. Este esquema resulta en un dispositivo más compacto, de menor costo y consumo de energía que considerando un sistema formado por moduladores de seis puertos. En particular, se implementó un prototipo capaz de controlar la envolvente compleja y la polarización de la señal transmitida. Para ello se utilizó una antena de dos puertos capaz de transmitir ondas electromagnéticas de polarización lineal ortogonales (vertical y horizontal). Todo el sistema se impelemntó para operar a una frecuencia central de $1575,42 \mathrm{MHz}$ utilizando como cargas variables diodos PIN controlados por corriente. Como ejemplo de aplicación, se generó una modulación 16-QAM para diferentes estados de polarización. Sin embargo, se mostró que el prototipo puede ser utilizado para obtener cualquier estado de salida utilizando un procedimiento de calibración para la determinación de las señales de banda base que configuran las cargas variables. Además, se estudió la dependencia entre las impedancias variables y las características de eficiencia y pérdidas por retorno del modulador. El diseño propuesto presentó una eficiencia baja de $35 \%$ y altas pérdidas por retorno. Por lo tanto, se analizó la eficiencia de los moduladores de seis puertos para compararla con el dispositivo propuesto y se mostró que, en general, la eficiencia teórica de estos cicuitos es inferior a $50 \%$. Como consecuencia, ambos dispositivos se utilizan en etapas de baja potencia y requieren de amplificadores en la salida para obtener los niveles de señal adecuados.

Una desventaja del prototipo presentado frente a los dispositivos de seis puertos es el alto valor de pérdidas por retorno. Por lo tanto, con el objetivo de mejorar esta característica, se estudió, implementó y midió una nueva impedancia variable basada en la interconexión de dos diodos PIN polarizados en directa. De acuerdo con el modelo teórico, el prototipo puede producir coeficientes de reflexión con una cobertura de fase completa dentro del círculo de $|\Gamma|=0,35$ en un rango de frecuencias de 1, 5-1, $6 \mathrm{GHz}$. Considerando otros diseños que logran un mayor área de cobertura, nuestro enfoque presentó un menor número de diodos, lo que resultó en un menor consumo de energía y menor número de señales de control. Finalmente, para obtener los valores de impedancia deseados se requiere de un procedimiento de calibración que estime los valores de las señales de control. Debido a la relación no lineal de las cargas se propusó utilizar un método basado en la técnica 
de aprendizaje por variedades (manifold learning). Este enfoque de calibración, en lugar de explotar el modelo analítico del dispositivo cuyos parámetros son de alguna manera desconocidos, se basa en un algoritmo supervisado que aprende el modelo intrínseco de la carga a través de un conjunto de datos de entrenamiento. Partiendo del supuesto de que las impedancias variables están controladas por varios parámetros independientes, el objetivo del método es la recuperación de esos parámetros de control para establecer un conjunto de valores de impedancia deseados a una frecuencia de operación. Se evaluó el método con datos sintéticos y datos reales. Este último surge de mediciones tomadas del prototipo de la carga variable presentada anteriormente. Para ambos ejemplos, se demostró que el método de calibración puede recuperar con precisión las señales de control deseadas de la carga, incluso en el ejemplo práctico que resulta difícil modelar la respuesta de la carga.

Por último, otro de los dispositivos propuestos es un demodulador de múltiples puertos capaz de recuperar las señales recibidas por un arreglo de antenas. Se plantearon los conceptos teóricos para el diseño de demoduladores de $N$ puertos y a partir de las expresiones desarrolladas se planteó un esquema de demodulación heterodino basado en la transformada de Hilbert. A modo de ejemplo, se diseñó un demodulador compuesto por una estructura de siete puertos conectada a tres antenas, tres detectores de potencia y un oscilador local. Se implementó el prototipo para operar a una frecuencia de 1575,42 MHz y a partir del mismo se validaron las expresiones analíticas. El circuito mostró una reducción significativa del costo y del consumo de energía del sistema respecto a implementaciones con arquitecturas de seis puertos, consecuencia directa del número de detectores de potencia utilizados. Sin embargo, también se produce un aumento de la complejidad del procesamiento digital, debido a la necesidad de implementar un filtro pasabanda y la transformada discreta de Hilbert.

Los sistemas de modulación y demodulación propuestos en esta tesis se basan en el concepto de seis puertos y al igual que los dispositivos de seis puertos establecen una alternativa de diseño que compite contra las arquitecturas convencional basadas en mezcladores, especialmente para frecuencias de operación que se encuentran en el espectro de microondas y ondas milimétricas, donde las dimensiones de los circuitos son similares a la longitud de onda de las señales. Se demostró que la utilización de estructuras de siete puertos brinda un hardware mucho mas simple que las alternativas de seis puertos, en términos de cantidad de componentes, costo de construcción y consumo de energía. Sin embargo, se produce un aumento en la complejidad del bloque digital, debido a la necesidad de utilizar un procesamiento más costoso en el demodulador y un método de calibración más complejo en el modulador. Gracias a los avances de los circuitos digitales en relación a la velocidad y capacidad de procesamiento, las arquitecturas propuestas establece una alternativa factible para el diseño de arreglos de sensores, que podría dezplazar a las arquitecturas de seis, especialmente cuando el sistema requiere de funciones de estimación de ángulo de arribo y/o polarización, además de la función de comunicaciones.

\section{Trabajo Futuro}

Existen muchas líneas de investigación que pueden continuarse más allá de los resultados mostrados en esta tesis, así como resultados parciales sobre los prototipos que requieren mayor confirmación teórica y experimental. En primer lugar, es importante realizar mediciones sobre los prototipos con antenas utilizando una cámara anecoica de RF y microondas. Esta es un recintos de ensayo en cuyo interior se trata de reproducir las 
condiciones de transmisión/recepción del espacio libre, evitando reflexiones en su interior con material absorbente, permitiendo realizar una caracterizacion adecuada del campo electromagnético.

Por otro lado, continua la búsqueda de nuevas alternativas de diseño de impedancias variables con el objetivo de aumentar la eficiencia y reducir las pérdidas por retorno del modulador de siete puertos. Además, el procedimiento de calibración propuesto para controlar una carga variable, puede extenderse al modulador completo.

Respecto al demodulador de siete puertos, los resultados se procesaron utilizando funciones que provee el software MATLAB, como la transformada discreta de Hilbert. Sin embargo, esta función podría implementarse en forma más eficiente considerando que la señal procesada es del tipo pasabanda. En consecuencia, un trabajo futuro consiste en la implementación de los algoritmos de procesamiento teniendo en cuenta las etapas de filtrado y la transformación de Hilbert. Los conceptos teóricos se desarrollaron para un diseño genérico de $N$ puertos, dejando abierta la búsqueda de nuevas arquitecturas con mayor cantidad de puerto (mayor que siete) para incorporar más antenas. 


\section{Apéndice A}

\section{Eficiencia de Conversión en Función de $\theta$}

Partiendo de la expresión (3.19) se calcula la magnitud cuadrado de los términos $\Gamma_{4}+\Gamma_{5}$ y $\Gamma_{6}+\Gamma_{7}$

$$
\begin{aligned}
\left|\Gamma_{4}+\Gamma_{5}\right|^{2} & =4|C|^{2}(1-\sin (\pi \sin (\theta))) \\
\left|\Gamma_{6}+\Gamma_{7}\right|^{2} & =4|C|^{2}(1+\sin (\pi \sin (\theta)))
\end{aligned}
$$

Notar que si $0^{\circ}<\theta<30^{\circ}$ entonces $\left|\Gamma_{6}+\Gamma_{7}\right|>\left|\Gamma_{4}+\Gamma_{5}\right|$, por el contrario si $-30^{\circ}<\theta<0^{\circ}$ entonces $\left|\Gamma_{4}+\Gamma_{5}\right|>\left|\Gamma_{6}+\Gamma_{7}\right|$. En consecuencia, a partir de la expresión de la eficiencia

$$
\eta=\frac{1}{8}\left(\left|\Gamma_{4}+\Gamma_{5}\right|^{2}+\left|\Gamma_{6}+\Gamma_{7}\right|^{2}\right)
$$

se plantean dos casos: si $\theta$ varia entre $0<\theta<30^{\circ}$ se toma como factor común el término $\rho=\left|\Gamma_{6}+\Gamma_{7}\right|$ y la eficiencia resulta

$$
\eta=\frac{\rho^{2}}{8}\left(\frac{\left|\Gamma_{4}+\Gamma_{5}\right|^{2}}{\left|\Gamma_{6}+\Gamma_{7}\right|^{2}}+1\right) \quad 0^{\circ} \leq \theta \leq 30^{\circ}
$$

y utilizando (A.1) y (A.2) se obtiene

$$
\eta=\frac{\rho^{2} / 4}{1+\sin (\pi \sin (\theta))} \quad 0^{\circ} \leq \theta \leq 30^{\circ} .
$$

Sin embargo, cuando $\theta$ varia entre $-30^{\circ}<\theta<0^{\circ}$ se toma como factor común el término $\rho=\left|\Gamma_{4}+\Gamma_{5}\right|$ y la eficiencia resulta

$$
\eta=\frac{\rho^{2} / 4}{1-\sin (\pi \sin (\theta))} \quad-30^{\circ} \leq \theta \leq 0^{\circ} .
$$

Finalmente, las expresiones (A.5) y (A.6) se pueden escribir en forma compacta como

$$
\eta=\frac{\rho^{2} / 4}{1+\sin (\pi|\sin (\theta)|)} \quad-30^{\circ} \leq \theta \leq 30^{\circ} .
$$




\section{Apéndice B}

\section{Eficiencia de Conversión en Función de $\beta$}

La siguiente demostración de la eficiencia considera el ejemplo de diversidad polarimétrica, que resulta similar al caso mostrados en el Apendice A.

Partiendo de la expresión (3.24) se calcula la magnitud cuadrado de los términos $\Gamma_{4}+\Gamma_{5} \mathrm{y} \Gamma_{6}+\Gamma_{7}$

$$
\begin{aligned}
& \left|\Gamma_{4}+\Gamma_{5}\right|^{2}=4|C|^{2}(\cos \beta-\sin \beta)^{2} \\
& \left|\Gamma_{6}+\Gamma_{7}\right|^{2}=4|C|^{2}(\cos \beta+\sin \beta)^{2}
\end{aligned}
$$

Notar que si $0^{\circ}<\beta<45^{\circ}$ entonces $\left|\Gamma_{6}+\Gamma_{7}\right|>\left|\Gamma_{4}+\Gamma_{5}\right|$, por el contrario si $-45^{\circ}<\beta<0^{\circ}$ entonces $\left|\Gamma_{4}+\Gamma_{5}\right|>\left|\Gamma_{6}+\Gamma_{7}\right|$. En consecuencia, a partir de la expresión de la eficiencia

$$
\eta=\frac{1}{8}\left(\left|\Gamma_{4}+\Gamma_{5}\right|^{2}+\left|\Gamma_{6}+\Gamma_{7}\right|^{2}\right)
$$

se plantean dos casos: si $\beta$ varia entre $0<\beta<45^{\circ}$ se toma como factor común el término $\rho=\left|\Gamma_{6}+\Gamma_{7}\right|$ y la eficiencia resulta

$$
\eta=\frac{\rho^{2}}{8}\left(\frac{\left|\Gamma_{4}+\Gamma_{5}\right|^{2}}{\left|\Gamma_{6}+\Gamma_{7}\right|^{2}}+1\right) \quad 0^{\circ} \leq \beta \leq 45^{\circ}
$$

y utilizando (B.1) y (B.2) se obtiene

$$
\eta=\frac{\rho^{2} / 4}{1+2 \cos \beta \sin \beta} \quad 0^{\circ} \leq \beta \leq 45^{\circ} .
$$

Sin embargo, cuando $\beta$ varia entre $-45^{\circ}<\beta<0^{\circ}$ se toma como factor común el término $\rho=\left|\Gamma_{4}+\Gamma_{5}\right|$ y la eficiencia resulta

$$
\eta=\frac{\rho^{2} / 4}{1-2 \cos \beta \sin \beta} \quad-45^{\circ} \leq \beta \leq 0^{\circ} .
$$

Finalmente, las expresiones (B.5) y (B.6) se pueden escribir en forma compacta como

$$
\eta=\frac{\rho^{2} / 4}{1+2 \cos \beta \sin |\beta|} \quad-45^{\circ} \leq \beta \leq 45^{\circ} .
$$




\section{Apéndice $\mathrm{C}$}

\section{Calibración del Modulador de Siete Puertos}

En la práctica, existen imperfecciones en los circuitos, tales como el desequilibrio de amplitud y fase dentro de la red de siete puertos, que pueden desviar los resultados de su comportamiento ideal. Por lo tanto, se requiere un método de calibración para obtener las señales de control que establecen el modulador en un estado deseado. El método propuesto se emplea en dos etapas: una etapa de entrenamiento y otra de estimación. En la etapa de entrenamiento, el modelo del modulador de siete puertos se implementa utilizando los datos presentados en la Sección 3.1.1. Considerando (3.8), el modelo resulta

$$
\left[\begin{array}{l}
b_{2}(I) \\
b_{3}(I)
\end{array}\right] / a_{1}=\left[\begin{array}{l}
k_{1} \\
k_{6}
\end{array}\right]+\left[\begin{array}{cccc}
k_{2} & k_{3} & k_{4} & k_{5} \\
k_{7} & k_{8} & k_{9} & k_{10}
\end{array}\right] \gamma(I)
$$

donde $k_{j}$ con $j \in 1, \ldots, 10$ son amplitudes complejas, $k_{1}$ y $k_{6}$ modelan el desbalance en los valores de las salidas debidos a la aislacion no ideal entre el puerto de entrada $P_{1}$ y las salidas $P_{2}$ y $P_{3}$. Además, $\gamma(I)$ es un vector columna que contiene los cuatro coeficientes de reflexión controlado por las corrientes de polarización $\left[\Gamma_{4}\left(i_{4}\right), \ldots, \Gamma_{7}\left(i_{7}\right)\right]^{T}$. Cada valor de $\Gamma_{j}\left(i_{j}\right)$ con $j=\{4,5,6,7\}$, es una función no lineal de $i_{j}$. Dicha función se aproxima por la interpolación lineal de los valores medidos presentados en la Fig. 3.6. Por lo tanto, se aplica el operador transmpuesta en (C.1) para obtener

$$
\left[b_{2}\left(I_{n}\right), b_{3}\left(I_{n}\right)\right] / a_{1}=\left[\begin{array}{ll}
1 & \gamma\left(I_{n}\right)^{T}
\end{array}\right] K^{T}
$$

donde $n$ define el estado, y $K \in \mathbb{C}^{2 \times 5}$ es una matriz compuesta por lo parámetros $k_{j}$. Agrupando $\operatorname{los} N=600$ estados de salida medidos y los correspondientes valores de los coeficientes de reflexión, se logra

$$
B=H K^{T}
$$

donde $B \in \mathbb{C}^{N \times 2}$ contiene los estados de salida, y $H \in \mathbb{C}^{N \times 5}$ se compone de un vector unitario (en la primer columna) y los valores de los coeficientes de reflexión. Entonces, los parámetros en $K$ se pueden obtener aplicando la técnica de minimos cuadrados

$$
\hat{K}^{T}=\left(H^{H} H\right)^{-1} H^{H} B .
$$


Por último, la etapa de estimación consiste de obtener las corrientes de control que generan los estados de salida $\overline{b_{2}}$ y $\overline{b_{3}}$, minimizando la siguiente función

$$
E\left(I_{n}\right)=\sqrt{\frac{\left|\overline{b_{2}^{n}}-b_{2}\left(I_{n}\right)\right|^{2}+\left|\overline{b_{3}^{n}}-b_{3}\left(I_{n}\right)\right|^{2}}{\left|\overline{b_{2}^{n}}\right|^{2}+\left|\overline{b_{3}^{n}}\right|^{2}}} .
$$

donde $n$ indica el número de estado. 


\section{Apéndice D}

\section{Expresión de la Salida del Filtro}

En este apéndice, se describen las expresiones utilizadas para obtener la señal a la salida del filtro pasabajo (6.15) partiendo de la potencia instantanea

$$
p_{i}(t) \approx K_{0}+K_{1}\left(\sum_{n=1}^{K+1} \operatorname{Re}\left\{s_{i n} a_{n}(t)\right\}\right)+K_{2}\left(\sum_{n=1}^{K+1} \operatorname{Re}\left\{s_{i n} a_{n}(t)\right\}\right)^{2} .
$$

La operación realizada por el filtro pasabajos se puede representar como una operación lineal, por lo tanto, se puede aplicar la operación a cada término de la potencia (D.1) por separado. En primer lugar, la constante $K_{0}$ permanece invariante debido a que se encuentra en la banda de paso del filtro. El segundo término relacionado a la constante $K_{1}$ será filtrado debido a que esta compuesto señales centradas a las frecuencias $f_{r}$ y $f_{c}$ $\left(a_{n}(t)=x_{n}(t) e^{j 2 \pi f_{c} t}\right.$ si $n \neq 1$, y $\left.a_{1}(t)=x_{1} e^{j 2 \pi f_{r} t}\right)$. Por último, se analiza el termino cuadratico determinando que componentes seran filtradas y cuales no. Para ello, se reescribe su expresión desarrollando los productos de la siguiente manera

$$
\begin{aligned}
K_{2}\left(\sum_{n=1}^{K+1} \operatorname{Re}\left\{s_{i n} a_{n}(t)\right\}\right)^{2} & =K_{2}\left(\sum_{n=1}^{K+1} \operatorname{Re}\left\{s_{i n} a_{n}(t)\right\}\right)\left(\sum_{k=1}^{K+1} \operatorname{Re}\left\{s_{i k} a_{k}(t)\right\}\right) \\
& =2 K_{2}\left(\sum_{n=1}^{K+1} \sum_{k=n+1}^{K+1} \operatorname{Re}\left\{s_{i n} a_{n}(t)\right\} \operatorname{Re}\left\{s_{i k} a_{k}(t)\right\}\right)+K_{2} \sum_{n=1}^{K+1} \operatorname{Re}\left\{s_{i n} a_{n}(t)\right\}^{2} .
\end{aligned}
$$

Además, los terminos de la sumatoria se pueden escribir como

$$
\begin{aligned}
\operatorname{Re}\left\{s_{i n} a_{n}(t)\right\} \operatorname{Re}\left\{s_{i k} a_{k}(t)\right\}= & \frac{\left(s_{i n} a_{n}(t)+s_{i n}^{*} a_{n}^{*}(t)\right)}{2} \frac{\left(s_{i k} a_{k}(t)+s_{i k}^{*} a_{k}^{*}(t)\right)}{2} \\
= & \frac{1}{4}\left(s_{i n} s_{i k} a_{n}(t) a_{k}(t)+s_{i n}^{*} s_{i k}^{*} a_{n}^{*}(t) a_{k}^{*}(t)\right. \\
& \left.+s_{i n} s_{i k}^{*} a_{n}(t) a_{k}^{*}(t)+s_{i n}^{*} s_{i k} a_{n}^{*}(t) a_{k}(t)\right) \\
= & \frac{1}{2}\left(\operatorname{Re}\left\{s_{i n} s_{i k} a_{n}(t) a_{k}(t)\right\}+\operatorname{Re}\left\{s_{i n} s_{i k}^{*} a_{n}(t) a_{k}^{*}(t)\right\}\right)(\mathrm{D} .4)
\end{aligned}
$$

y si $n=k$ entonces

$$
\operatorname{Re}\left\{s_{i n} a_{n}(t)\right\}^{2}=\frac{1}{2}\left(\operatorname{Re}\left\{\left(s_{i n} a_{n}(t)\right)^{2}\right\}+\left|s_{i n} a_{n}(t)\right|^{2}\right) .
$$


Por lo tanto, utilizando (D.4) y (D.5) en (D.2) se obtiene

$$
\begin{aligned}
K_{2}\left(\sum_{n=1}^{K+1} \operatorname{Re}\left\{s_{i n} a_{n}(t)\right\}\right)^{2}= & K_{2} \sum_{n=1}^{K+1} \sum_{k=n+1}^{K+1}\left(\operatorname{Re}\left\{s_{i n} s_{i k} a_{n}(t) a_{k}(t)\right\}+\operatorname{Re}\left\{s_{i n} s_{i k}^{*} a_{n}(t) a_{k}^{*}(t)\right\}\right) \\
& +\frac{K_{2}}{2} \sum_{n=1}^{K+1}\left(\operatorname{Re}\left\{\left(s_{i n} a_{n}(t)\right)^{2}\right\}+\left|s_{i n} a_{n}(t)\right|^{2}\right) .
\end{aligned}
$$

Al aplicar el filtro pasabajos, los término $\operatorname{Re}\left\{s_{i n} s_{i k} a_{n}(t) a_{k}(t)\right\}$ y $\operatorname{Re}\left\{\left(s_{i n} a_{n}(t)\right)^{2}\right\}$ serán eliminados debido a que se encuentra en frecuencias $f_{r}+f_{c}, 2 f_{r}$ y $2 f_{c}$. Por el contrario, si la frecuencia de corte del filtro es mayo que $\left|f_{r}-f_{c}\right|$, los términos $\operatorname{Re}\left\{s_{i n} s_{i k}^{*} a_{n}(t) a_{k}^{*}(t)\right\}$ y $\left|s_{i n} a_{n}(t)\right|^{2}$ pemanecen invariantes dado que se encuentran en banda base y en $\left|f_{r}-f_{c}\right|$. La salida del filtro resulta

$$
\begin{aligned}
y_{i}(t) & =L P\left\{p_{i}(t)\right\} \\
& =K_{0}+\frac{K_{2}}{2} \sum_{n=1}^{K+1}\left|s_{i n} a_{n}(t)\right|^{2}+K_{2} \sum_{n=1}^{K+1} \sum_{k=n+1}^{K+1} \operatorname{Re}\left\{s_{i n} s_{i k}^{*} a_{n}(t) a_{k}^{*}(t)\right\} .
\end{aligned}
$$

Para obtener exactamente la expresión (6.15) se reescribe el segundo termino extrayendo la sumatoria correspondiente a $n=1$, lo cual resulta

$$
\begin{aligned}
y_{i}(t)= & K_{0}+\frac{K_{2}}{2} \sum_{n=1}^{K+1}\left|s_{i n} a_{n}(t)\right|^{2}+K_{2} \sum_{n=2}^{K+1} \sum_{k=n+1}^{K+1} \operatorname{Re}\left\{s_{i n} s_{i k}^{*} a_{n}(t) a_{k}^{*}(t)\right\} \\
& +K_{2} \sum_{n=1}^{K+1} \operatorname{Re}\left\{s_{i 1} s_{i n}^{*} a_{1}(t) a_{n}^{*}(t)\right\} .
\end{aligned}
$$




\section{Bibliografía}

[1] Y. Ding and K. Wu, "Half-mode substrate integrated waveguide six-port front-end circuits for direct-conversion transceiver design," in 2008 IEEE MTT-S International Microwave Symposium Digest, San Diego, CA, Jun 2008, pp. 1175-1178.

[2] Bryant, G. H., Principles of Microwave Measurements. Peter Peregrinus/ IEE, 1993.

[3] D. Tse and P. Viswanath, Fundamentals of Wireless Communication, 4th ed. CAMBRIDGE UNIVERSITY PRESS, Feb 2017.

[4] S.-G. Zhou, G.-L. Huang, T.-H. Chio, J.-J. Yang, and G. Weik, "Design of a wideband dual-polarization full-corporate waveguide feed antenna array," IEEE Transactions on Antennas and Propagation, vol. 63, no. 11, pp. 4775-4782, Sep 2015.

[5] H. Nawaz and I. Tekin, "Dual polarized patch antenna with high inter-port isolation for 1ghz in-band full duplex applications," in 2016 IEEE International Symposium on Antennas and Propagation (APSURSI). IEEE, Oct 2016, pp. 1947-1491.

[6] Grau, A. and Romeu, J. and Blanch, S. and Jofre, L. and Jafarkhani, Hamid and De Flaviis, F., "Performance enhancement of the Alamouti diversity scheme using polarization-reconfigurable antennas in different fading environments," in Antennas and Propagation Society International Symposium 2006. IEEE, Jul 2006, pp. 133136.

[7] Sirianunpiboon, S. and Howard, S. D. and Calderbank, A. R., "Diversity gains across line of sight and rich scattering environments from space-polarization-time codes," in Information Theory for Wireless Networks, 2007 IEEE Workshop on Information Theory, Jul 2007.

[8] Y. M. M. Santalla, V.and Antar and A. G. Pino, "Polarimetric radar covariance matrix algorithms and applications to meteorological radar data," IEEE Transactions on Geoscience and Remote Sensing, vol. 37, no. 2, pp. 1128-1137, Mar 1999.

[9] F. Lang, J. Yang, D. Li, L. Zhao, and L. Shi, "Polarimetric sar image segmentation using statistical region merging," IEEE Geoscience and Remote Sensing Letters, vol. 11, no. 2, pp. 509-513, Feb 2014.

[10] R. Iglesias, D. Monells, X. Fabregas, J. J. Mallorqui, A. Aguasca, and C. LopezMartinez, "Phase quality optimization in polarimetric differential sar interferometry," IEEE Transactions on Geoscience and Remote Sensing, vol. 52, no. 5, pp. 2875-2888, May 2014. 
[11] M. Pöpperl, J. Adametz, and M. Vossiek, "Polarimetric radar barcode: a novel chipless rfid concept with high data capacity and ultimate tag robustness," IEEE Transactions on Microwave Theory and Techniques, vol. 64, no. 11, pp. 3686-3694, Sep 2016.

[12] D. H. Johnson and D. E. Dudgeon, Array signal processing: concepts and techniques. Prentice Hall, 1993.

[13] V.-Y. Vu, A. Judson Braga, X. Begaud, and B. Huyart, "Measurement of directionof-arrival of coherent signals using five-port reflectometers and quasi-yagi antennas," IEEE Microwave and Wireless Components Letters, vol. 15, no. 9, pp. 558-560, Sep 2005.

[14] J. Moghaddasi, T. Djeraf, and K. Wu, "Multiport interferometer-enabled 2-d angle of arrival (aoa) estimation system," IEEE Transactions on Microwave Theory and Techniques, vol. 65, no. 5, pp. 1767-1779, May 2017.

[15] J. G. Proakis and M. Salehi, Communication Systems Engineering, 2nd ed. Upper Saddle River, N.J: Pearson, Aug. 2001.

[16] J. Osth, Owais, M. Karlsson, A. Serban, and S. Gong, "Direct carrier six-port modulator using a technique to suppress carrier leakage," IEEE Transactions on Microwave Theory and Techniques, vol. 59, no. 3, pp. 741-747, Mar 2011.

[17] A. J. Venere, M. Hurtado, and C. H. Muravchik, "Sintesis de polarización con redes de siete puertos," in XVI Reunión de Trabajo en Procesamiento de la Información y Control, RPIC, Oct. 2015.

[18] A. J. Venere, M. Hurtado, R. L. La Valle, and C. H. Muravchik, "New Design of a Variable Impedance Based on Polarized Diodes at Microwave Frequency," IEEE Microwave and Wireless Components Letters, vol. 27, no. 5, pp. 470-472, May 2017.

[19] A. J. Venere, M. Hurtado, and C. H. Muravchik, "Calibration of nonlinear variable loads based on manifold learning," in XVII Reunión de Trabajo en Procesamiento de la Información y Control, RPIC, Sep. 2017.

[20] A. J. Venere, M. Hurtado, R. L. La Valle, and C. H. Muravchik, "Design of a multiport microwave modulator for dynamic polarization reconfiguration," IEEE Microwave and Wireless Components Letters, vol. 27, no. 5, pp. 470-472, May 2017.

[21] A. J. Venere, M. Hurtado, and C. H. Muravchik, "Polarimetric demodulator based on a seven-port network," in IEEE ARGENCON, 2018., Jun. 2018.

[22] — , "Design of a seven-port demodulator for wireless applications with multiple antennas," in XVI Reunión de Trabajo en Procesamiento de la Información y Control, RPIC, Sep. 2019.

[23] G. Engen and C. Hoer, "Application of an arbitrary 6-port junction to powermeasurement problems," IEEE Transactions on Instrumentation and Measurement, vol. 21, no. 4, pp. 470-474, Nov 1972. 
[24] C. A. Hoer, "The six-port coupler: A new approach to measuring voltage, current, power, impedance, and phase," IEEE Transactions on Instrumentation and Measurement, vol. 21, no. 4, pp. 466-470, Nov 1972.

[25] Hoer, C. A. and Roe, K. C., "Using an arbitrary six-port junction to measure complex voltage ratios," IEEE Transactions on Microwave Theory and Techniques, vol. 23, pp. 978-984, Dec 1975.

[26] C. A. Hoer, "A network analyzer incorporating two six-port reflectometers," IEEE Transactions on Microwave Theory and Techniques, vol. 25, pp. 1070-1074, Dec 1977.

[27] G. Engen, "The six-port reflectometer: An alternative network analyzer," IEEE Transactions on Microwave Theory and Techniques, vol. 25, no. 12, pp. 1075-1080, Dec 1977.

[28] A. L. De Souza Rolim, A. J. Belfort de Oliveira, and M. T. De Melo, "Six-port complex permittivity measurements," Sep 2006, pp. 492-494.

[29] M. E. Bialkowski and G. S. Woods, "Design and analysis off a six-port receiver for use in near-field antenna measurements," vol. 134, no. 6, Dec 1987, pp. 515-520.

[30] A. Mahmoodshahi and W. K. Kahn, "Polarization measurement using six-port network analizer: A theoretical and experimental approach," in Digest on Antennas and Propagation Society International Symposium, Jun 1989, pp. 1018-1021.

[31] J. Li, R. G. Bosisio, and K. Wu, "A six-port direct digital millimeter wave receiver," in Microwave Symposium., vol. 3. IEEE, May 1994, pp. 1659-1662.

[32] B. Hnyart, J.-J. Laurin, R. G. Bosisio, and D. Roscoe, "A direction-finding antenna system using an integrated six-port circuit," IEEE Transactions on Antennas and Propagation, vol. 4, no. 12, pp. 1508-1512, Dec 1995.

[33] Y. Zhao, C. Viereck, J. Frigon, R. Bosisio, and K. Wu, "Direct quadrature phase shift keying modulator using six-port technology," Electronics Letters, vol. 41, no. 21, pp. $1180-1181$, oct 2005 .

[34] J. Osth, M. Karlsson, A. Serban, and S. Gong, "M-QAM six-port modulator using only binary baseband data, electrical or optical," IEEE Transactions on Microwave Theory and Techniques, vol. 61, no. 6, pp. 2506-2513, Jun 2013.

[35] S. O. Tatu, E. Moldovan, K. Wu, and R. Bosisio, "A new direct millimeter-wave six-port receiver," IEEE Transactions on Microwave Theory and Techniques, vol. 49, no. 12, pp. 2517-2522, Dec 2001.

[36] X. Xu, R. Bosisio, and K. Wu, "A new six-port junction based on substrate integrated waveguide technology," IEEE Transactions on Microwave Theory and Techniques, vol. 53, no. 3, pp. 2267-2272, Jul 2005.

[37] E. Moldovan, S. O. Tatu, T. Gaman, K. Wu, and R. Bosisio, "A new 94 ghz sixport collision avoidance radar sensor," IEEE Transactions on Microwave Theory and Techniques, vol. 52, no. 3, pp. 751-759, Mar 2004. 
[38] Ghannouchi, Fadhel M. and Mohammadi, Abbas, The six-port technique with microwave and wireless applications, ser. Artech House microwave library. Artech House, 2009.

[39] Pozar, David M., Microwave Engineering, 2nd ed. John Wiley \& Sons, 1998.

[40] A. Koelpin, G. Vinci, B. Laemmle, D. Kissinger, and R. Weigel, "The six-port in modern society," IEEE Microwave Magazine, vol. 11, no. 7, pp. 35-43, Dec 2010.

[41] T. Hentschel, "The six-port as a communications receiver," IEEE Transactions on Microwave Theory and Techniques, vol. 53, no. 3, pp. 1039-1047, Mar. 2005.

[42] A. Serban, J. Östh, M. Karlsson, Owais, S. Gong, J. Haartsen, and P. Karlsson, "Sixport transceiver for 6-9 ghz ultra-wideband systems," IEEE Microwave and Optical Technology Letters, vol. 52, no. 3, pp. 740-746, Mar 2010.

[43] J. Osth, M. Karlsson, A. Serban, and S. Gong, "Schottky diode as high-speed variable impedance load in six-port modulators," in Ultra-Wideband (ICUWB), 2011 IEEE International Conference on. IEEE, 2011, pp. 68-71.

[44] — - "Carrier leakage suppression and EVM dependence on phase shifting network in six-port modulator," in Microwave and Millimeter Wave Technology (ICMMT), 2012 International Conference on, vol. 4. IEEE, 2012, pp. 1-4.

[45] A. Serban, M. Karlsson, J. Osth, Owais, and S. Gong, "Differential circuit technique for six-port modulator and demodulator," in 2012 IEEE/MTT-S International Microwave Symposium Digest, vol. 5, 2012, pp. 6-8.

[46] J. Osth, A. Serban, M. Karlsson, and S. Gong, "Lo leakage in six-port modulators and demodulators and its suppression techniques," in 2012 IEEE/MTT-S International Microwave Symposium Digest, vol. 2, no. 4, 2012, pp. 1-3.

[47] Y. Zhao, J. Frigon, K. Wu, and R. Bosisio, "Multi(six)-port impulse radio for ultrawideband," IEEE Transactions on Microwave Theory and Techniques, vol. 54, no. 4, pp. 1707-1712, Jun 2003.

[48] H. Mott, Remote Sensing with Polarimetric Radar. John Wiley and Sons, Dec 2006.

[49] K.-O. Sun, M. K. Choi, and D. V. D. Weide, "A pin diode controlled variable attenuator using a 0-db branch-line coupler," IEEE Microwave and Wireless Components Letters, vol. 15, no. 6, pp. 440-442, Jun 2005.

[50] J. K. Hunton and A. G. Ryals, "Microwave variable attenuators and modulators using pin diodes," IRE Transactions on Microwave Theory and Techniques, vol. 10, no. 4, pp. 262-273, Jul 1962.

[51] M. Tsuji, T. Nishikawa, K. Wakino, and T. Kitazawa, "Bi-directionally fed phasedarray antenna downsized with variable impedance phase shifter for ism band," IEEE Transactions on Microwave Theory and Techniques, vol. 54, no. 7, pp. 2962-2969, Jul 2006. 
[52] H. M. Nemati, C. Fager, U. Gustavsson, R. Jos, and H. Zirath, "Design of varactorbased tunable matching networks for dynamic load modulation of high power amplifiers," IEEE Transactions on Microwave Theory and Techniques, vol. 57, no. 5, pp. 1110-1118, May 2009.

[53] J. Li, J. Jiang, Y. He, W. Xu, M. Chen, L. Miao, and S. Bie, "Design of a tunable lowfrequency and broadband radar absorber based on active frequency selective surface," IEEE Antennas and Wireless Propagation Letters, vol. 15, pp. 774-777, Aug 2015.

[54] B. M. Albinsson, H. Guo, M. Schoon, and H. O. Vickes, "A new programmable load for noise parameter determination," IEEE Transactions on Microwave Theory and Techniques, vol. 39, no. 2, pp. 216-223, Feb 1991.

[55] V. F. Pochiraju, T.and Fusco, J. Francey, and H. Schmassmann, "Low-power variable impedance load/tuning unit," Electronics Letters, vol. 44, no. 21, pp. 216-223, Oct 2008.

[56] R. R. Coifman and S. Lafon, "Diffusion maps," Applied and Computational Harmonic Analysis, vol. 21, no. 1, pp. 5-30, Jul. 2006. [Online]. Available: http://www.sciencedirect.com/science/article/pii/S1063520306000546

[57] R. Talmon, D. Kushnir, R. R. Coifman, I. Cohen, and S. Gannot, "Parametrization of Linear Systems Using Diffusion Kernels," IEEE Transactions on Signal Processing, vol. 60, no. 3, pp. 1159-1173, Mar. 2012.

[58] J. Moghaddasi and K. Wu, "Improved joint radar-radio (radcom) transceiver for future intelligent transportation platforms and highly mobile high-speed communication systems," in IEEE International Wireless Symposium (IWS), 2013, pp. 1-4.

[59] — - "Multifunctional transceiver for future radar sensing and radio communicating data-fusion platform," in IEEE access, Feb 2016, pp. 818-838. 